Supporting Information for

\title{
Chiral Mutagenesis of Insulin.
}

Foldability and Function are Inversely Regulated by a Stereospecific Switch in the B Chain

Satoe H. Nakagawa, Ming Zhao, Qing-xin Hua, Shi-Quan Hu, Zhu-li Wan, Wenhua Jia, \&

Michael A. Weiss

\section{Table of Contents}

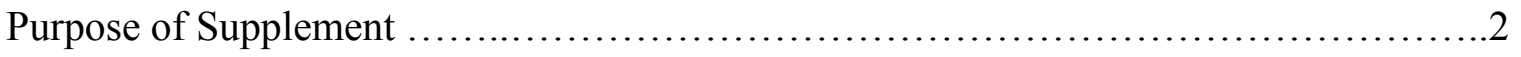

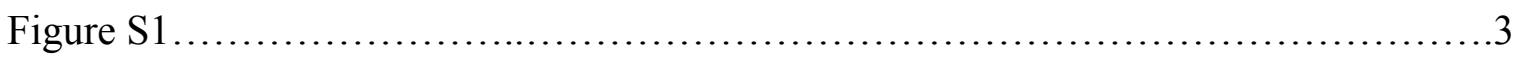

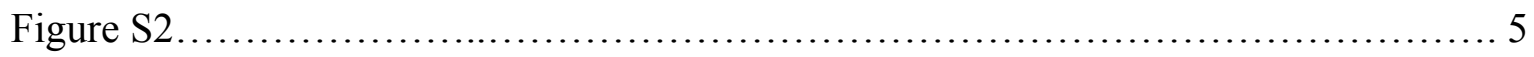

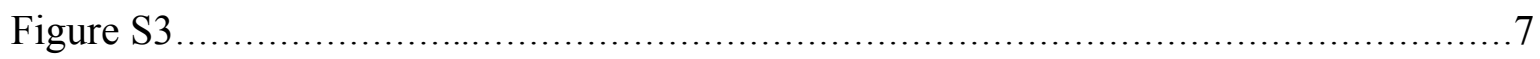

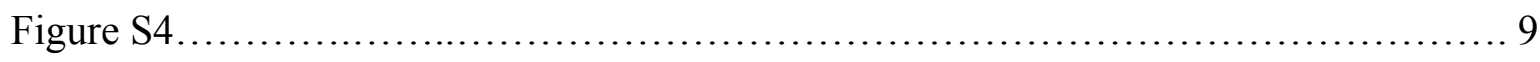

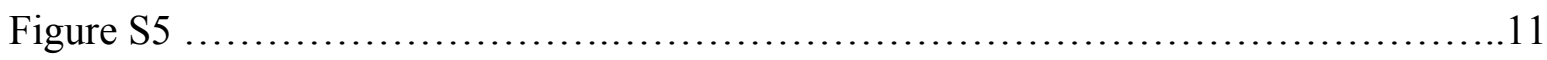

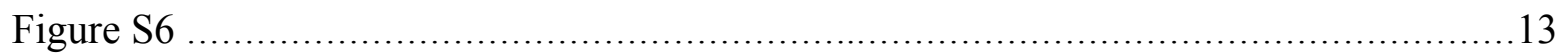

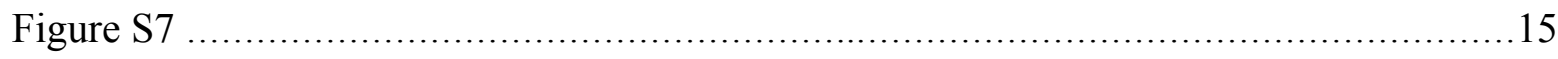

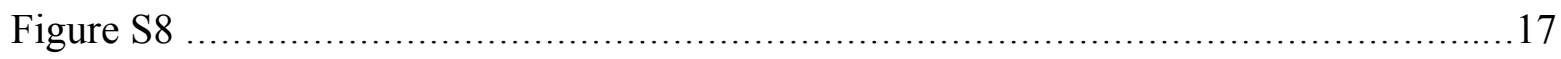

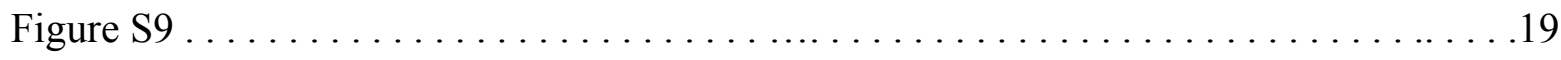

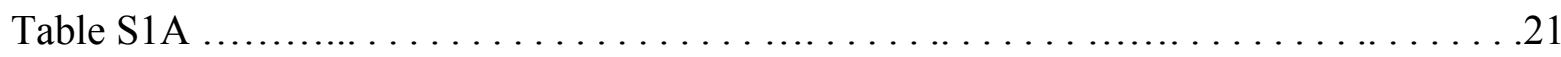

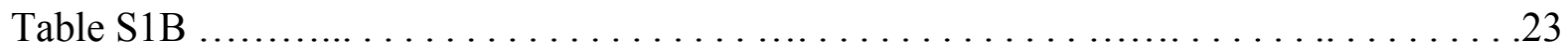

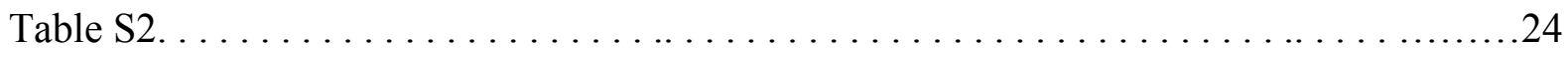

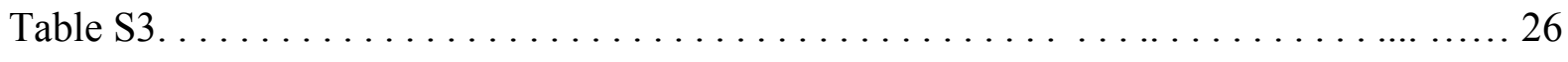

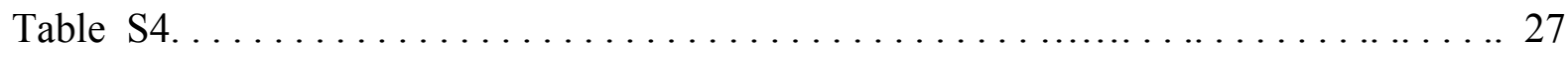

Table S5................................................29

Table S6.............................................. 30

Table S7.............................................. 31 


\section{Purpose of the Supplement}

Nine figures and nine tables are provided as Supplemental Material. These are included to clarify structural relationships (Fig. S1) or provide additional data (Fig. S2-S7 and supplemental tables S1-S6). Figure S2 illustrates the analysis of combinatorial B8 library of insulin analogues by HPLC and MALDI-TOF mass spectrometry. CD and 1D NMR spectra of D- and L-Ser ${ }^{\mathrm{B} 8}$ analogues are shown in Figure S3. Figure S4 provides additional 2D NMR spectra of D-Ala ${ }^{\mathrm{B} 8}$-DKP-insulin. Figure S5 compares TOCSY spectra of D-Ala ${ }^{\mathrm{B} 8}$-DKP-insulin and DKP-insulin. NMR analysis is summarized in Figures S6 and S7: a diagonal plot of interresidue NOEs (Fig. S6) and summary of sequential assignment in Wüthrich format (Fig. S7). Figure S8 illustrates the distinct geometries of the metal-binding sites in zinc insulin hexamers (predominantly octahedral in the $\mathrm{T}_{3}$-related trimer and tetrahedral in $\mathrm{R}_{3}$-related trimers) and their spectroscopic signature in optical absorption studies of cobalt hexamers. These data suggest that $\mathrm{D}-\mathrm{Ala}{ }^{\mathrm{B} 8}$-insulin is able to form an $\mathrm{R}$ state but that the TR transition is partially impaired relative to wild-type insulin. Figure S9 illustrates sites of substitution near B8 investigated in previous mutagenesis studies, the results of which are summarized in Figure S1. These data highlight how unusual -- and even extraordinary -- is the 1000-fold decrement in receptor binding exhibited by D-Ala ${ }^{\mathrm{B} 8}$ analogues. Table S2 provides a summary of unit-cell dimensions and space groups among previously described structures of zinc insulin hexamers in structural classes $T_{3} R_{3}{ }^{f}$ and $R_{6}$. The following supplemental tables (S3-S8) provide NMR resonance assignments and $\mathrm{DG} / \mathrm{RMD}$ restraints related to $\mathrm{D}-\mathrm{Ala}^{\mathrm{B}}{ }^{\mathrm{B}}$-DKP-insulin. 
Figure S1. Structural relationship between Gly ${ }^{\mathrm{B} 8}$ and the A7-B7 disulfide bridge. (A) Stereo pair showing overview of the three disulfide bridges in insulin (cystines A7-B7, A20-B19, and A6-A11; yellow) in relation to the position of $\mathrm{Gly}^{\mathrm{B} 8}$ (red) in the T state. The A chain is shown in black and the B chain in green. (B) Expansion of B8 region. Stereotopic $\alpha$-protons of Gly ${ }^{\mathrm{B} 8}$ are shown in magenta (pro-D) and blue (pro-L). L amino-acid substitutions at B8 impede formation of cystine A7-B7 in insulin chain combination (see text). 
A
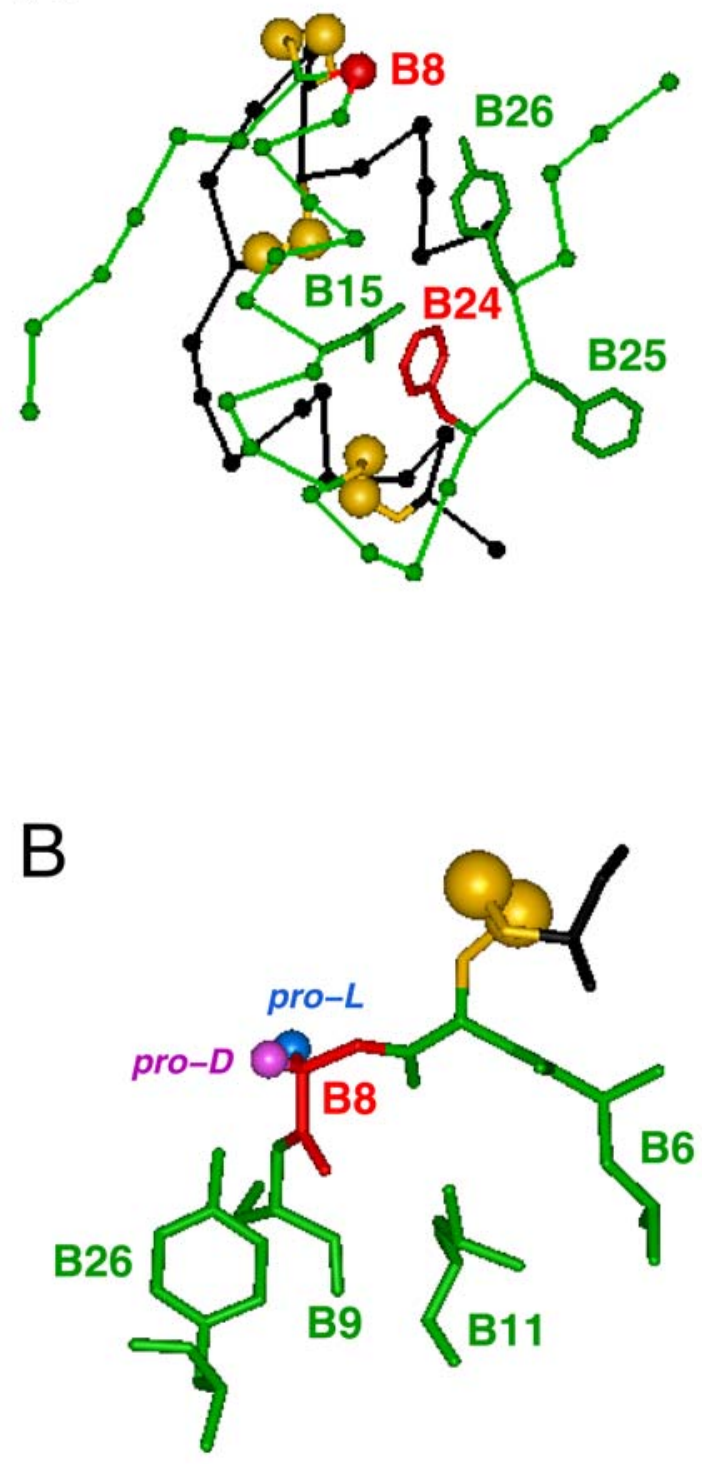
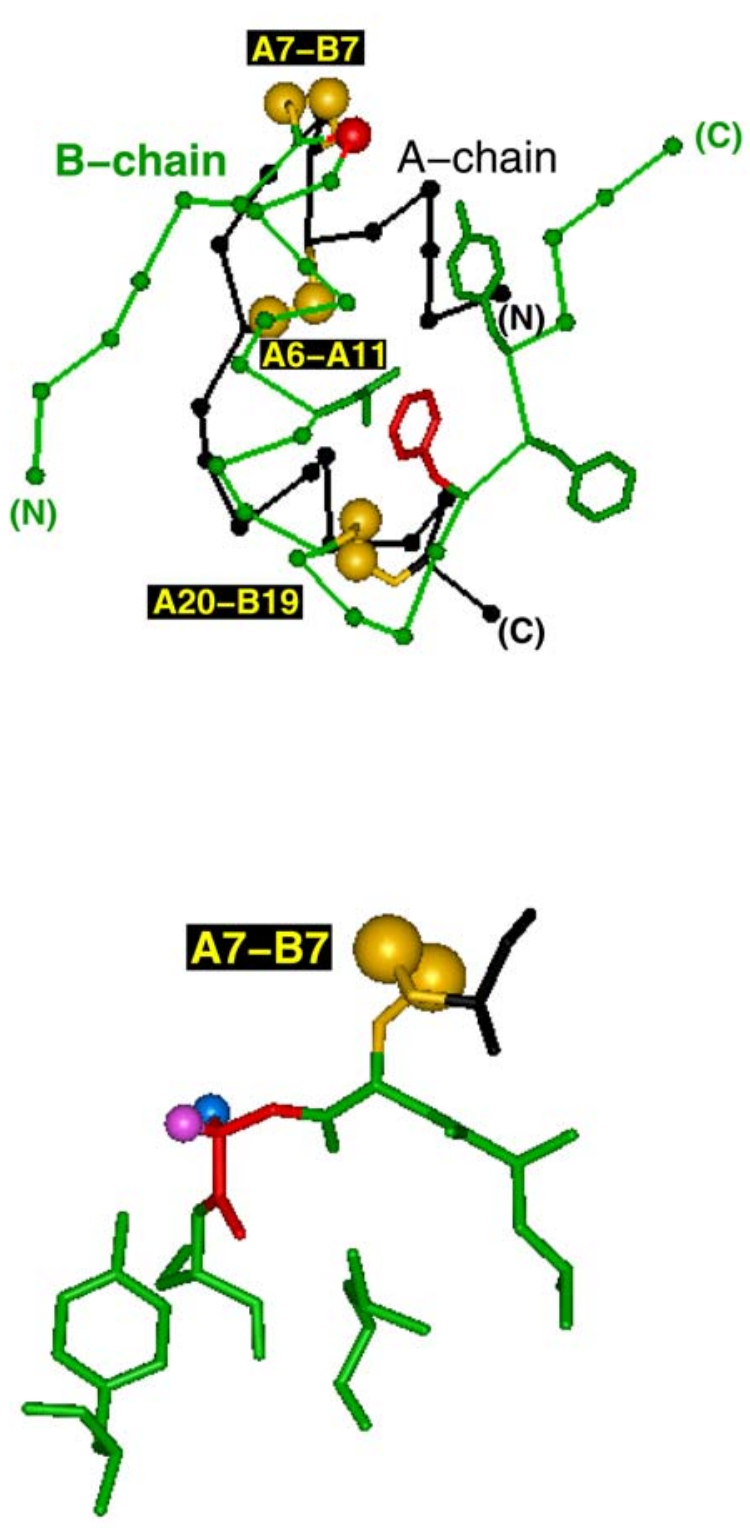

Figure S1 
Figure S2. Reverse-phase HPLC and MS analysis of D library of B8 analogues. (A) HPLC chromatogram. Eluted fractions a-j contain one or more B8 D analogues as indicated by MS analysis. (B) Receptor-binding studies of HPLC fractions. Representative displacement curves for HPLC fractions $a, b$ and $d$. Estimates of relative affinities are imprecise due to uncertainties in protein concentration and proportion of constituent analogues in mixtures. (C and D) Representative MALDI-TOF MS spectra of HPLC fractions a and $b$. Whereas fraction a contains a mixture of D-Ser ${ }^{\mathrm{B} 8}$-DKP-insulin and D-Tyr ${ }^{\mathrm{B} 8}$-DKP-insulin, fraction $\mathrm{b}$ contains $\mathrm{a}$ single predominant species, D-Arg ${ }^{\mathrm{B} 8}$-DKP-insulin. 

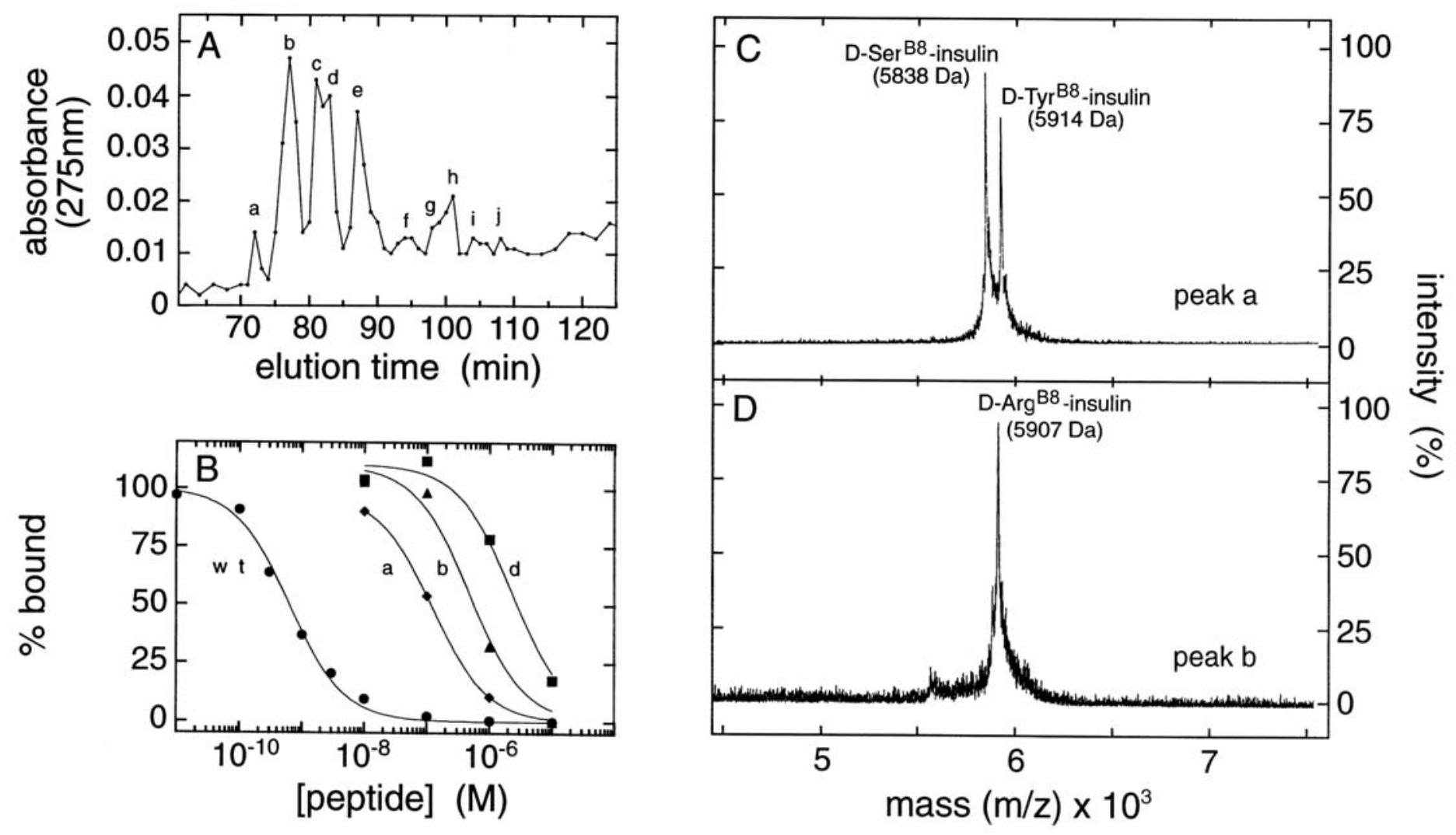

Figure S2 
Figure S3. Structural analysis of D- and L-Ser ${ }^{\mathrm{B} 8}$ analogues of DKP-insulin. (A) Far-UV CD spectra of B8 analogues: $\mathrm{Gly}^{\mathrm{B} 8}$ (solid line), $\mathrm{L}-\mathrm{Ser}^{\mathrm{B} 8}$ (open circles), and D-Ser ${ }^{\mathrm{B} 8}$ (solid circles). (B) Corresponding guanidine denaturation studies. (C) One-dimensional ${ }^{1} \mathrm{H}-\mathrm{NMR}$ spectra at $\mathrm{pH}$ 7.4 and $25^{\circ} \mathrm{C}$ : (a) L-Ser ${ }^{\mathrm{B} 8}$; (b) D-Ser ${ }^{\mathrm{B} 8}$, and (c) parent spectrum of DKP-insulin. 

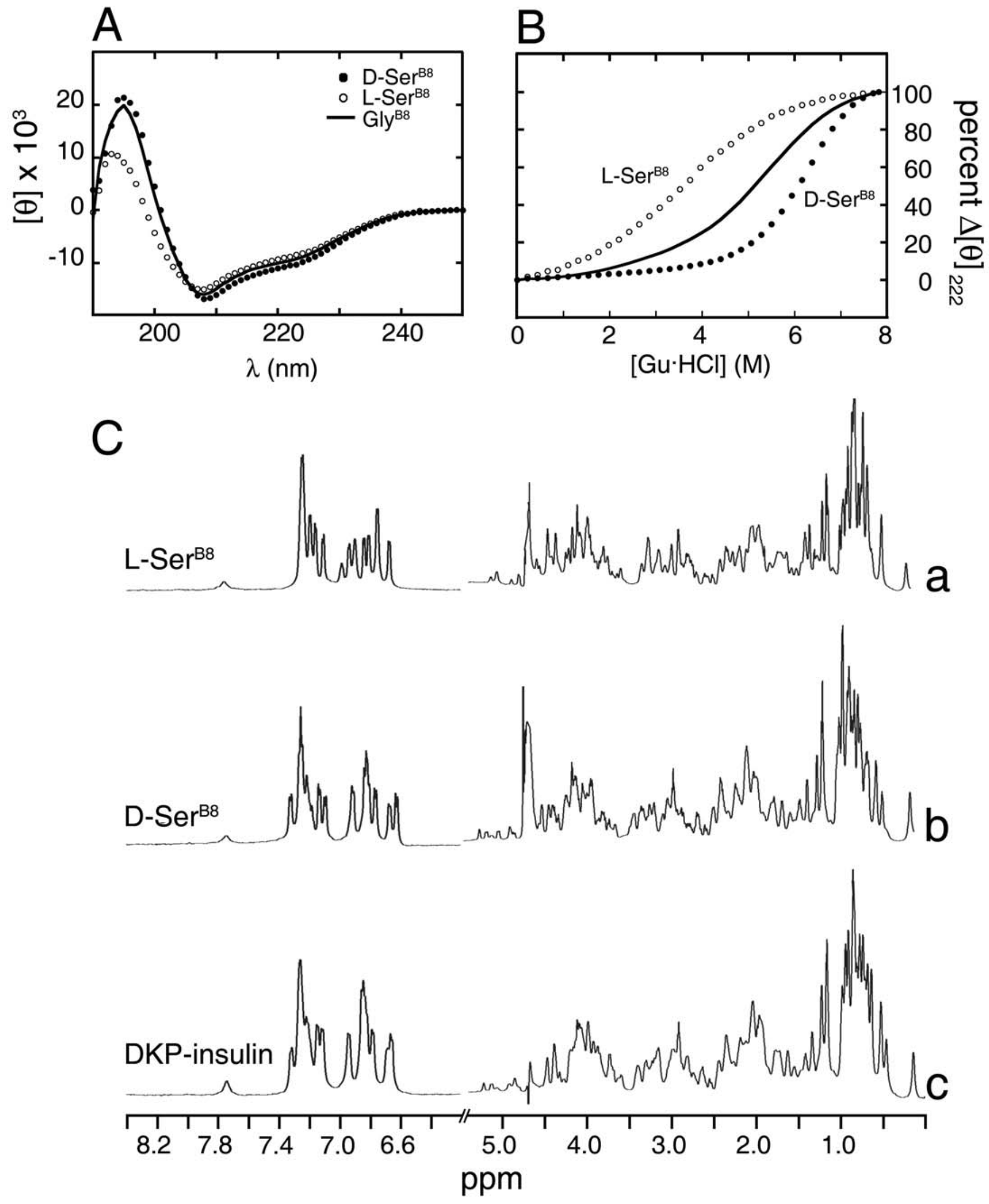

Figure S3 
Figure S4. ${ }^{1} \mathrm{H}-\mathrm{NMR}$ NOESY spectrum of D-Ala ${ }^{\mathrm{B} 8}$-DKP-insulin in $\mathrm{H}_{2} \mathrm{O}\left(10 \% \mathrm{D}_{2} \mathrm{O}\right)$ at pH 7.4 and $25{ }^{\circ} \mathrm{C}$. (A) Cross-peaks between exchangeable and aromatic resonances $\left(\omega_{2}\right.$; vertical axis) and aliphatic resonances $\left(\omega_{1}\right.$; horizontal axis). Box in panel A is shown in expanded form in (B): NOEs to the novel methyl resonance of $\mathrm{D}-\mathrm{Ala}^{\mathrm{B} 8}$ from the amide resonances of Leu ${ }^{\mathrm{B} 11}$ (labeled a) and $\mathrm{Val}^{\mathrm{B} 12}(\mathrm{~b})$. The $\mathrm{B} 12 \mathrm{H}_{\mathrm{N}}$ also exhibits a native-like NOE to the methyl resonance of Ala ${ }^{\mathrm{B} 14}$ (c). (C) For comparison, the NOESY spectrum of DKP-insulin exhibits no detectable NOEs to the $\mathrm{H}_{\alpha}$ resonances of $\mathrm{Gly}^{\mathrm{B} 8}$ from the amide resonances of B11 or B12 (positions X within empty box). Intra-residue $\mathrm{H}_{\mathrm{N}}-\mathrm{H}_{\alpha}$ cross-peaks $\mathrm{d}$ and e are assigned to $\mathrm{B} 11$ and B12, respectively (Hua, Q. X., et al. ((1996) J. Mol. Biol. 259, 297-313). NOESY spectra were acquired at $600 \mathrm{MHz}$ with a mixing time of $200 \mathrm{~ms}$. 

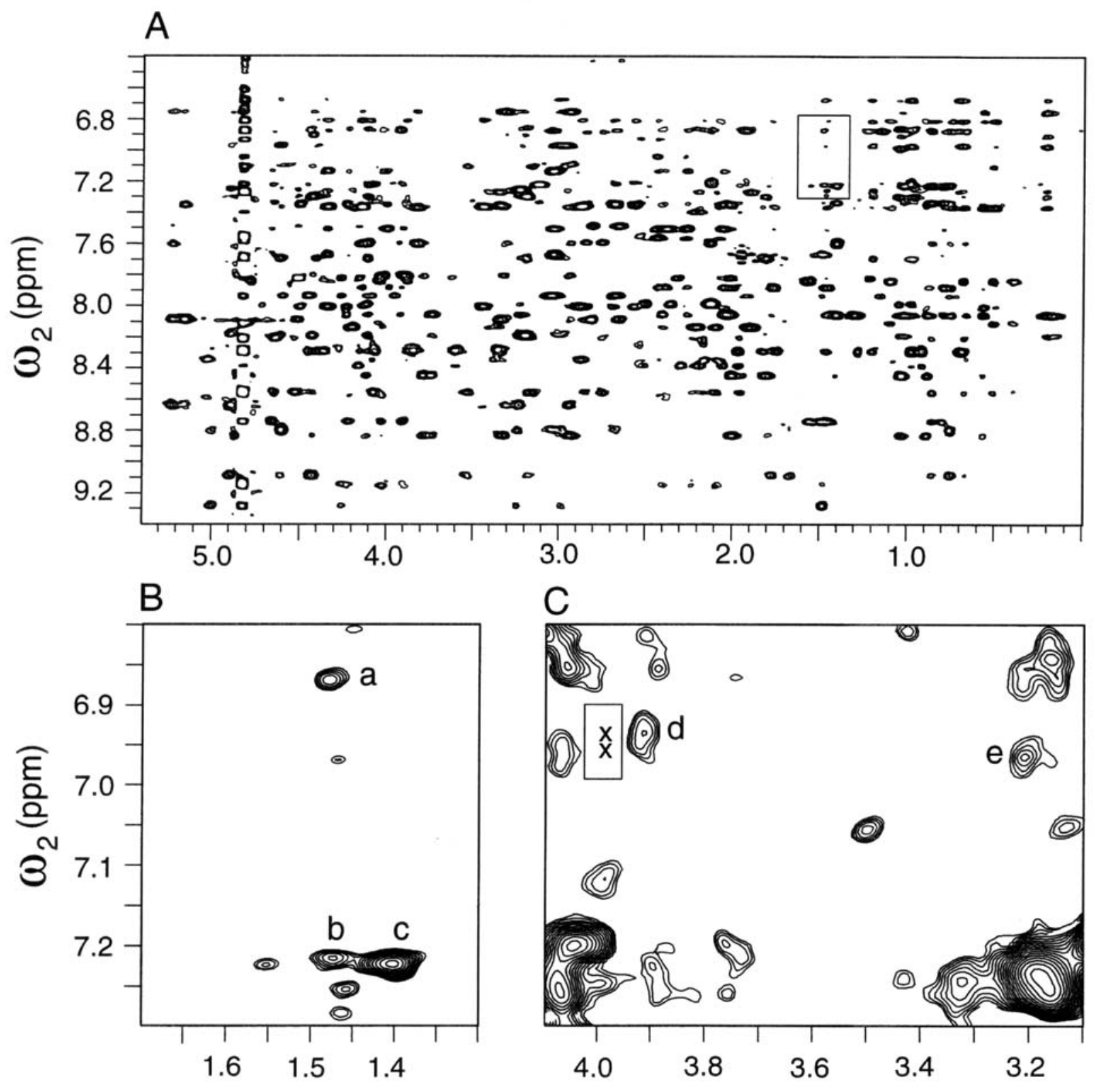

locked variant

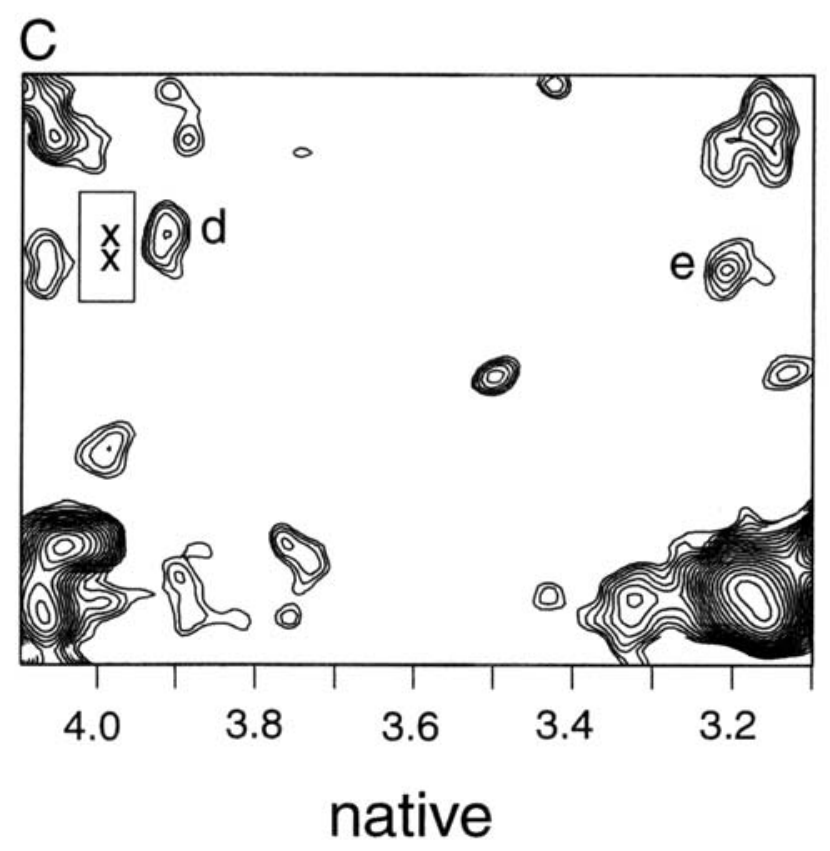

Figure S4 
Figure S5. D-Ala ${ }^{\mathrm{B} 8}$-DKP-insulin exhibits greater protection of amide protons than does DKPinsulin in $20 \%$ deuterioacetic acid and $80 \% \mathrm{D}_{2} \mathrm{O}(\mathrm{pH} 1.9)$ at $25{ }^{\circ} \mathrm{C}$. Initial TOCSY spectra of (A) DKP-insulin and (B) D-Ala ${ }^{\mathrm{B} 8}$ analogue following initial dissolution. Peaks are as labeled. The mixing time was $55 \mathrm{~ms}$. Data were obtained between 20 minutes and 2 hours and 50 minutes after dissolving the powder. 

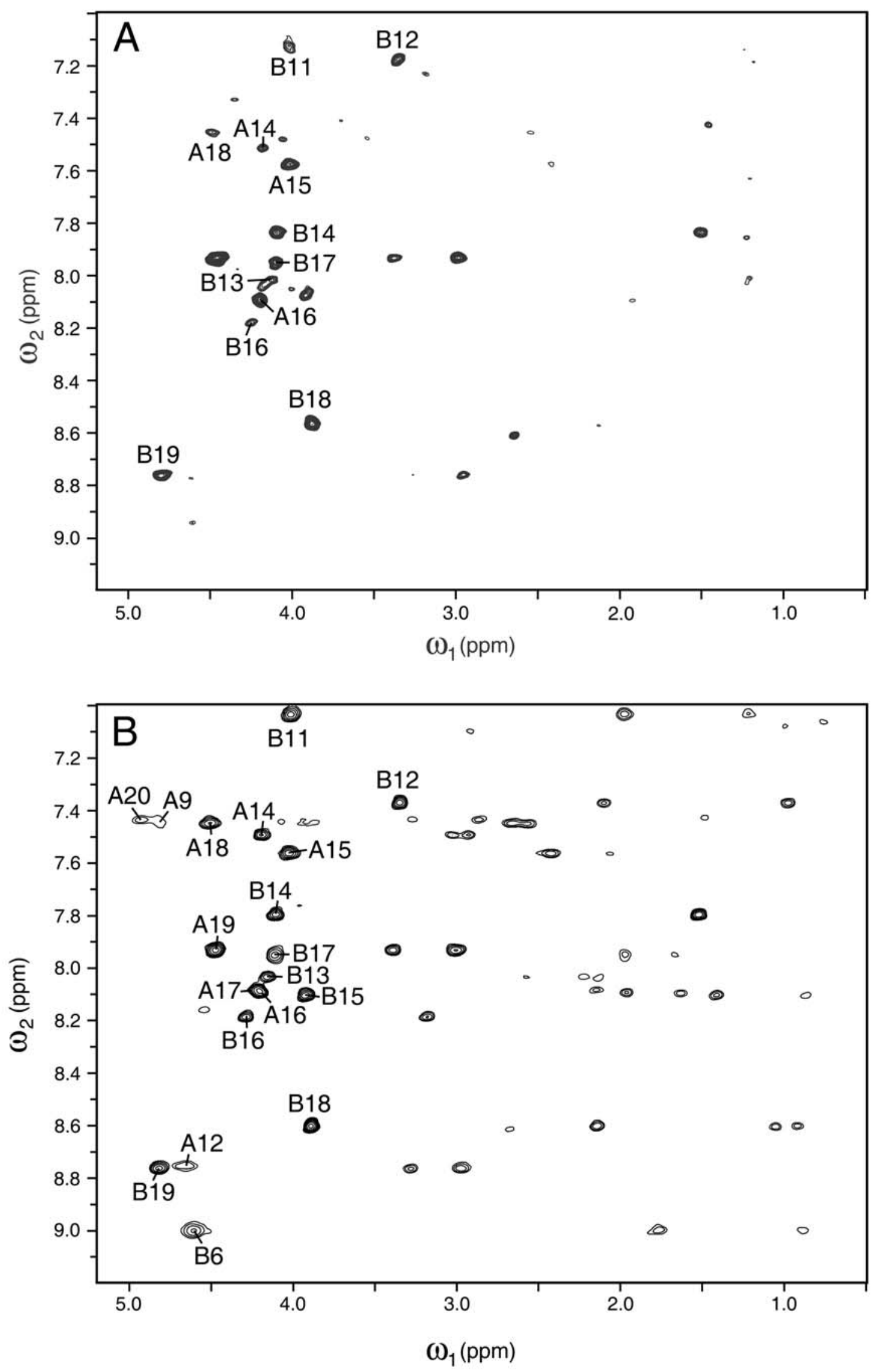

Figure S5 
Figure S6. Diagonal plot of inter-residue contacts in NOESY spectra of D-Ala ${ }^{\mathrm{B} 8}$-DKP-insulin. NOEs between side chains are shown at lower right; NOEs between main-chain protons or between main chain and side chains are shown at upper left. Red boxes indicate NOEs consistent with crystal structures of T-state protomers but weak or unobserved in spectra of parent DKP-insulin analogue (Hua, Q. X., et al. (1996) J. Mol. Biol. 259, 297-313): (a) A3-B7, (b) A7-B11, (c) A21-B23, (d) A2-B26, (e) A6-B11, f) A11-B6, and (g) A21-B19. Data were obtained both at neutral $\mathrm{pH}$ in aqueous solution and in $20 \%$ deuterioacetic acid as described (Hua, Q. X., et al. (1996) J. Mol. Biol. 259, 297-313). 


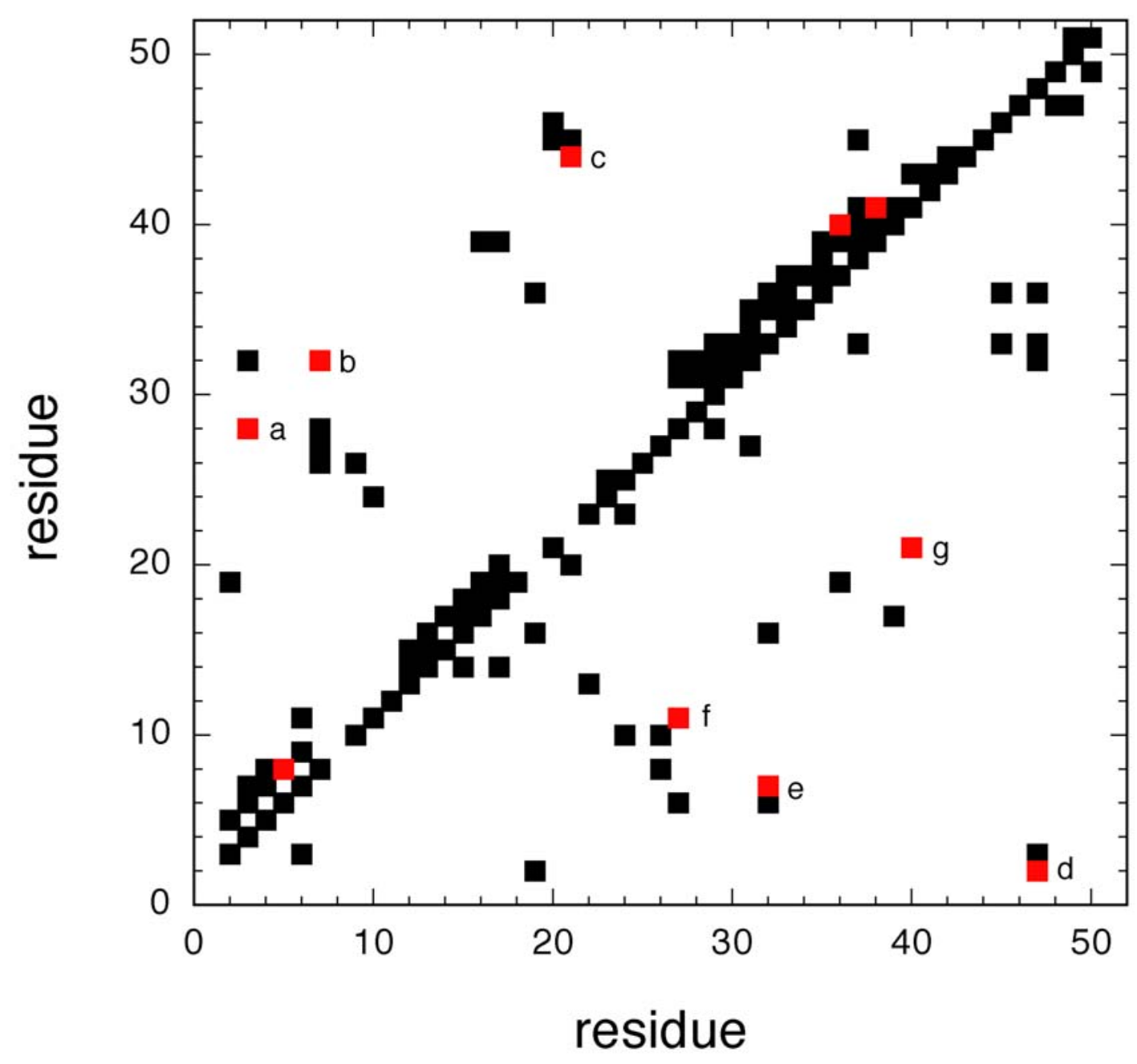

Figure S6 
Figure S7. NMR analysis of D-Ala ${ }^{\mathrm{B} 8}$-DKP-insulin: summary of sequential assignment and protect amide resonances in Wüthrich format. The location and extent of $\alpha$-helices are in accord with crystallographic T-state protomers and previous NMR studies of DKP-insulin: (upper panel) A chain and (lower panel) B chain. Analysis of protected amide resonances in $\mathrm{D}_{2} \mathrm{O}$ solution (open circles beneath amino-acid sequence) was performed in $20 \%$ deuterioacetic acid as described (Hua, Q. X., et al. (1996) J. Mol. Biol. 259, 297-313). Above B-chain sequence asterisk indicates site of $\mathrm{D}-\mathrm{Ala}^{\mathrm{B} 8}$ substitution, and arrows indicate the three sites of substitution in DKP template $\left(\mathrm{His}^{\mathrm{B} 10} \rightarrow \mathrm{Asp}, \mathrm{Pro}^{\mathrm{B} 29} \rightarrow\right.$ Lys, and $\mathrm{Lys}^{\mathrm{B} 29} \rightarrow$ Pro). 


$$
\begin{aligned}
& d_{\alpha N} \\
& d_{\beta N} \\
& d_{N N} \\
& d_{\alpha N(i, i+3)} \\
& d_{\alpha \beta(i, i+3)} \\
& d_{\alpha \beta(i, i+3)} \\
& d_{\alpha N(i, i+4)} \\
& d_{\alpha N(i, i+3)} \\
& d_{N N}
\end{aligned}
$$$$
\text { G I VEQ C C T S I C S L Y Q L E N Y C N }
$$

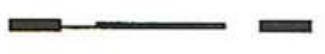$$
-
$$
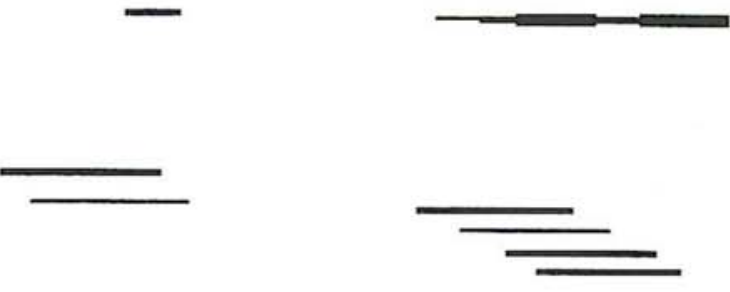

$* \quad \frac{1}{\nabla}$

FV N Q H L C A S D L V V E A

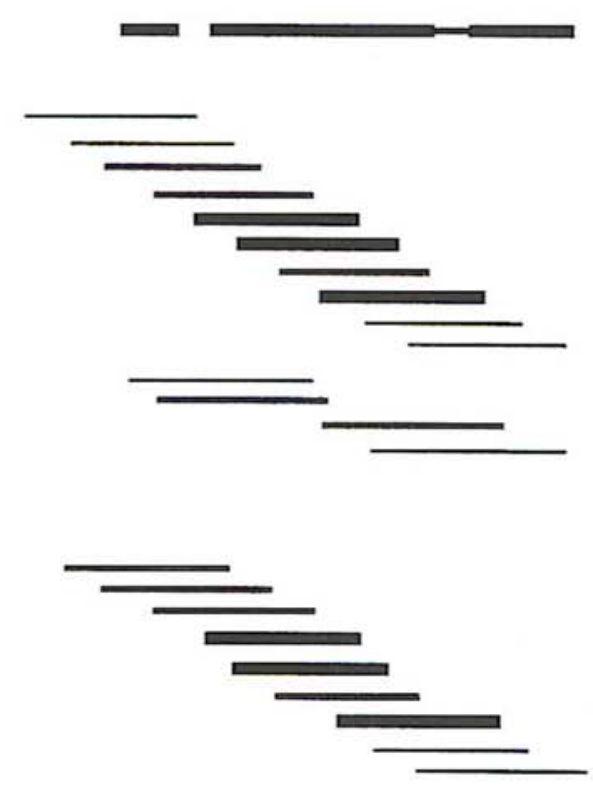

Figure S7 
Figure S8. D-Ala ${ }^{\mathrm{B} 8}$ substitution permits formation of an $\mathrm{R}$ state but partially blocks the TR transition.

(A and B) Molecular structures (stereo pairs) of zinc-binding sites in representative $\mathrm{T}_{6}$ hexamer (A) and $R_{6}$ hexamer $(B)$. Whereas the $T_{3}$-related site is predominantly octahedral, the $R_{3}$-related site is tetrahedral. This difference has been exploited to monitor the TR transition in $\mathrm{Co}^{2+}$ substituted hexamers in wild-type insulin (Karatas, Y., Kruger, P., \& Wollmer, A. (1991) Biol Chem Hoppe Seyler. 372, 1035-1038) and among insulin analogues (Nakagawa, S. H., Tager, H. S. (1991) J. Biol. Chem. 266, 11502-11509).

(C and D) Comparison of TR transitions of wild-type insulin in $\mathrm{Co}^{2+}$-substituted hexamers (C) and D-Ala ${ }^{\mathrm{B} 8}$-insulin in $\mathrm{Co}^{2+}$-substituted hexamers (D). The presence of cobalt $\mathrm{d}$-d bands near $550 \mathrm{~nm}$ provides a signature of the $\mathrm{R}$ state; its attenuation in the $\mathrm{D}-\mathrm{Ala}{ }^{\mathrm{B} 8}$ analogue indicates a perturbated or incomplete TR transition. Solid and dashed lines indicate the presence or absence of $0.8 \mathrm{M} \mathrm{NaSCN}$. X-ray crystallographic analysis of zinc insulin hexamers obtained under conditions (C) indicate $\mathrm{R}_{6}$ structures whereas crystallographic analysis of $\mathrm{D}-\mathrm{Ala}{ }^{\mathrm{B} 8}$-insulin hexamers obtained under conditions (D) depict $T_{3} R_{3}^{f}$ structures (Z. Wan and M. A. Weiss, manuscript in preparation). Blockage of the TR transition at the intermediate stage gives rise to a 1:1 combination of octahedral and tetrahedral sites, rationalizing the near-50\% attenuation of the cobalt $\mathrm{d}$-d bands in (D). Spectra were acquired in a buffer consisting of $50 \mathrm{mM}$ Tris-HCl ( $\mathrm{pH}$ 8), $0.07 \mathrm{mM} \mathrm{ZnCl}_{2}$, and $50 \mathrm{mM}$ phenol. The concentration of insulin or insulin analogue was $0.2 \mathrm{mM}$.

These results are consistent with model peptide studies of DeGrado and coworkers showing that D-Ala can be incorporated into the middle of an $\alpha$-helix but with a free-energy price of about 1 $\mathrm{kcal} / \mathrm{mole}$ relative to L-Ala (Fairman, R., Anthony-Cahill, S. J., \& DeGrado, W. (1992) J. Am. Chem. Soc. 114, 5458-5459). By analogy, the D-Ala ${ }^{\mathrm{B} 8}$ substitution in insulin, although stabilizing the T state, can also be accommodated in an R-state-specific $\alpha$-helix but at a cost. The high ligand concentrations ( $50 \mathrm{mM}$ phenol) employed to trigger the TR transition in the zinc hexamer can pay the price for only a half-transition. 


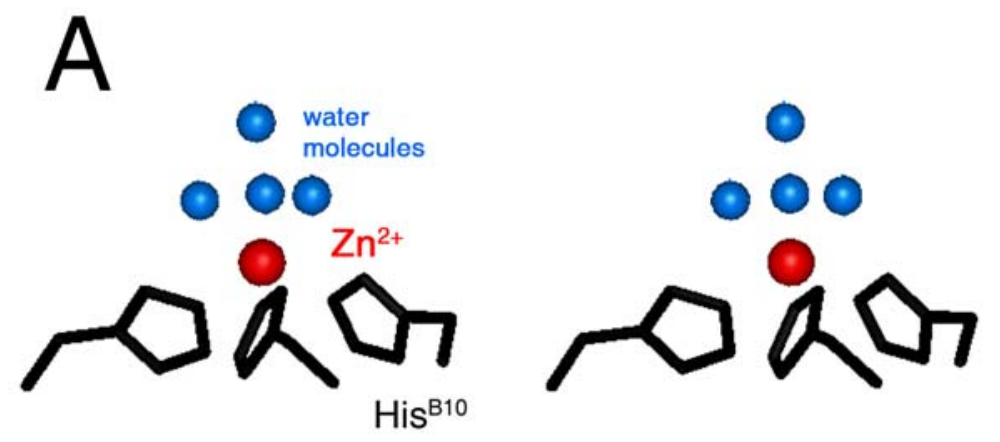

octahedral ( $\mathrm{T}$ )

B
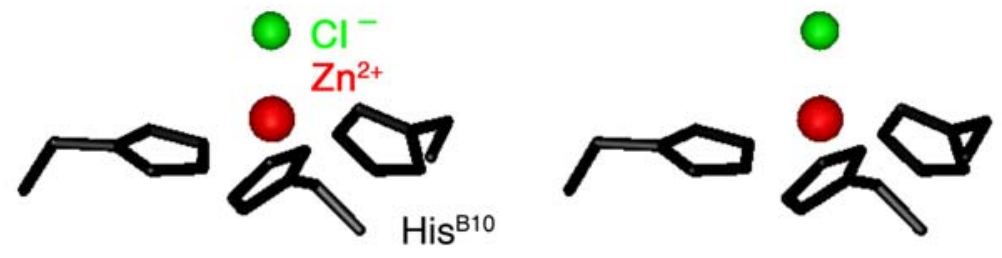

tetrahedral $(R)$

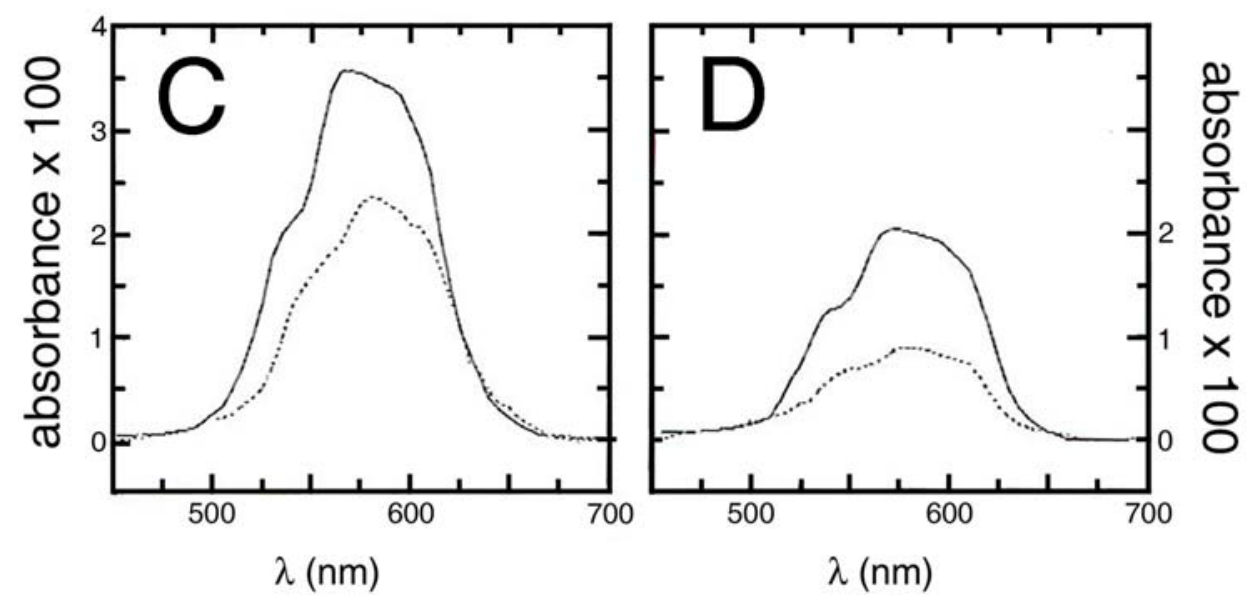

Figure S8 
Figure S9. Survey of sites near B8 that have previously been probed by mutagenesis. The 1000 -fold decrement in receptor binding exhibited by D-AlaB8-insulin is significantly more marked than that observed in mutational studies of these neighboring sites (see Table S1 for relative activities and references). We hypothesize that whereas previous mutations have probed favorable or unfavorable local contacts, incurring only modest effects on binding, D-Ala ${ }^{\mathrm{B} 8}$ impairs binding by a different mechanism: chiral obstruction of induced fit.

Since the active conformation of insulin is unknown, three representative structures are shown: (A) the $\mathrm{T}$ state (PDB identifier 4INS), (B) $\mathrm{R}$ state (PDB identifier 1EV6) and (C) despentapeptide[B26-B30]-insulin (DPI; PDB identifier 1PID). Highlighted positions (in green) are A8, B5, B6, B9, B10, B12, and B13. Color code: the All other side-chains and backbones are shown in grey. Structures are aligned with respect to residues A2-A8, A13-A19 and B9-B19. Molecules are represented in CPK model. 


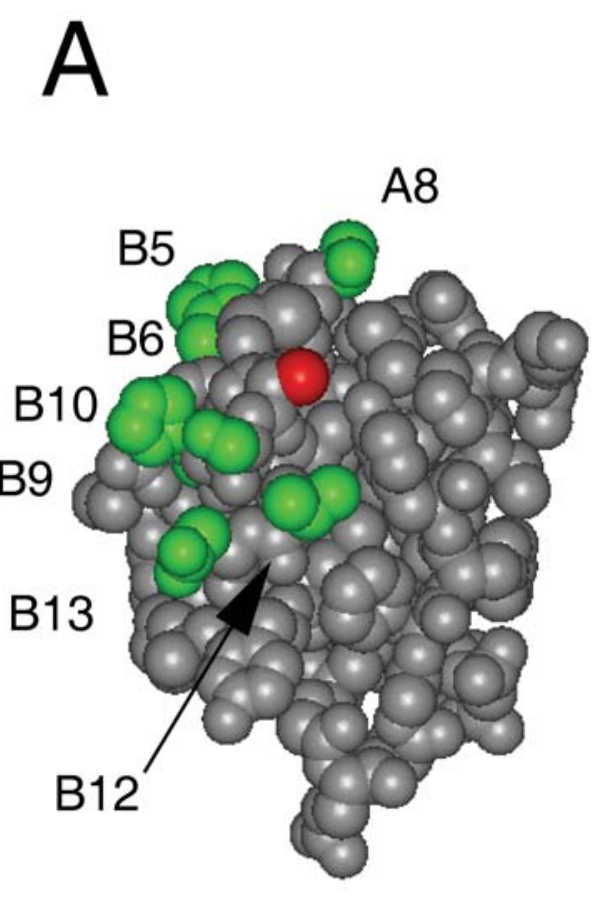

B

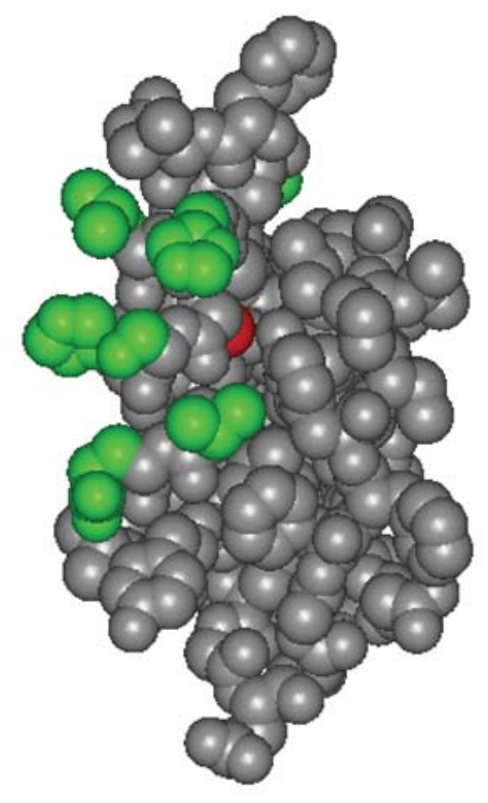

Figure S9
C

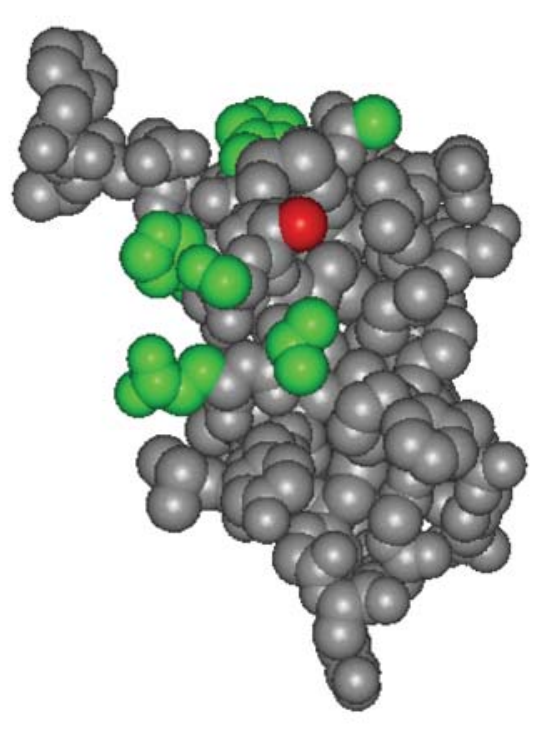


Table S1A (biological acvities). Relative Affinities of Insulin Analogs Surrounding B8 position ${ }^{\mathrm{a}}$.

\begin{tabular}{|c|c|c|c|}
\hline analog & activity & analog & activity \\
\hline A3 Val & & B9 Ser & \\
\hline $\mathrm{Ile}^{\mathrm{A} 3}$ & $11^{\mathrm{b}}$ & His ${ }^{\mathrm{B} 9}$ & $69^{\mathrm{i}}$ \\
\hline allo-Ile $\mathrm{A}^{\mathrm{A} 3}$ & $18^{\mathrm{b}}$ & $\operatorname{Asn}^{\mathrm{B} 9}$ & $47^{\mathrm{i}}$ \\
\hline tert-Leu ${ }^{\mathrm{A} 3}$ & $36^{\mathrm{b}}$ & B10 His & \\
\hline $\operatorname{Thr}^{\mathrm{A3}}$ & $13^{\mathrm{b}}, 50^{\mathrm{c}}$ & $\mathrm{Asp}^{\mathrm{B} 10}$ & $>200^{g}$ \\
\hline$A 8 T h r$ & & $\mathrm{Asn}^{\mathrm{B} 10}$ & $35^{\mathrm{j}}$ \\
\hline $\mathrm{Phe}^{\mathrm{A} 8}$ & $100^{\mathrm{d}}$ & B12 Val & \\
\hline $\mathrm{His}^{\mathrm{A} 8}$ & $>200^{\mathrm{e}}$ & $\mathrm{Ile}^{\mathrm{B} 12}$ & $13^{\mathrm{k}}$ \\
\hline $\mathrm{Leu}^{\mathrm{A} 8}$ & $>200^{\mathrm{f}}$ & allo-Ile ${ }^{\mathrm{B} 12}$ & $77^{\mathrm{k}}$ \\
\hline B5 His & & tert-Leu ${ }^{\mathrm{B} 12}$ & $36^{\mathrm{k}}$ \\
\hline $\mathrm{Asn}^{\mathrm{B} 5}$ & $46^{\mathrm{g}}$ & $\mathrm{Thr}^{\mathrm{B} 12}$ & $57^{1}$ \\
\hline $\mathrm{Ala}^{\mathrm{B} 5}$ & $31^{\mathrm{d}}$ & B13 Glu & \\
\hline B6 Leu & & $\mathrm{Gln}^{\mathrm{B} 13}$ & $18^{\mathrm{m}}$ \\
\hline $\mathrm{Phe}^{\mathrm{B} 6}$ & $10^{\mathrm{h}}$ & $\mathrm{Ala}^{\mathrm{B} 13}$ & $12^{\mathrm{n}}$ \\
\hline $\mathrm{Met}^{\mathrm{B} 6}$ & $15^{\mathrm{h}}$ & & \\
\hline
\end{tabular}

${ }^{a}$ Values are relative to human insulin, assumed to be $100 \%$ of the receptor binding affinity unless specified. ${ }^{b}$ Data were obtained from Nakagawa et al. (Biochem. 31, 3204-3214 (1992)). $\mathrm{N} \mathrm{Phe}{ }^{\mathrm{B} 1}, \mathrm{~N} \mathrm{Lys}{ }^{\mathrm{B} 29}$-bisacetyl-insulin was used as a template for all A3 mutations tested in this study, affinity results shown here is relative to this bisacetyl-insulin molecule, this template molecule has $76 \%$ binding affinity relative to insulin. ${ }^{c}$ Data were obtained from Wang et al. ((1996) Biochem. Mol. Biol. Int. 39, 1245-1254). ${ }^{\mathrm{d}}$ Data were obtained from Marki et al. ((1979) Physiol. Chem. 360, 1619-1632). ${ }^{\mathrm{e}}$ Data were obtained from Kaarsholm et al. ((1993) Biochem. 32, 10773-10778). ${ }^{\mathrm{f}} \mathrm{Data}$ were obtained from Katsoyannis et al. (unpublished results, personal communication, P.G. 
Katsoyannis). ${ }^{g}$ Data were obtained from Burke et al. ((1990) Biochem. Biophys. Res. Commun., 173, 982-987). ' Data were obtained from Nakagawa et al. ((1991) J. Biol. Chem. 266, 11502-11509). 'Data were obtained from Brange et al. ((1988) Nature. 333, 679-682), these values reflect the lipogenesis activities of these analogs. ${ }^{j}$ Data were obtained from ((1984) Int. J. Pept. Protein Res. 23, 394-401), these values reflect the lipogenesis activities of these analogs. ${ }^{\mathrm{k}}$ Data were obtained from Nakagawa et al. ((2000) Biochem. 39, 15826-15835). 'Data were obtained from Wang et al. ((1996) Biochem. Mol. Biol. Int. 39, 1245-1254). ${ }^{\mathrm{m}}$ Data were obtained from Markussen et al. ((1987) Protein Eng. 1, 205-213), these values reflect the lipogenesis activities of these analogs. ${ }^{\mathrm{n}}$ Data were obtained from Kristensen et al. ((1997) J. Biol. Chem. 272, 12978-12983). 
Table S1B. Distances between the $\mathrm{Ca}$ of $\mathrm{B} 8$ to the $\mathrm{Ca}$ of the neighboring residues.

\begin{tabular}{cccc}
\hline $\begin{array}{c}\text { Distance between the } \mathrm{C} \alpha \text { of } \mathrm{B} 8 \\
\text { to the } \mathrm{C} \alpha \text { of residue }(\mathrm{A})\end{array}$ & $\begin{array}{c}\text { T state (4INS) } \\
\text { (molecule 1) }\end{array}$ & DPI (1PID) & $\begin{array}{c}\text { R state (1EV6) } \\
\text { (molecule 2) }\end{array}$ \\
\hline Thr $^{\mathrm{A} 8}$ & 11.04 & 10.83 & 11.46 \\
$\mathrm{His}^{\mathrm{B} 5}$ & 10.21 & 10.18 & 5.16 \\
$\mathrm{Leu}^{\mathrm{B} 6}$ & 7.02 & 7.28 & 5.40 \\
$\mathrm{Ser}^{\mathrm{B} 9}$ & 3.73 & 3.79 & 3.80 \\
$\mathrm{His}^{\mathrm{B} 10}$ & 5.26 & 5.46 & 5.35 \\
$\mathrm{Val}^{\mathrm{B} 12}$ & 6.98 & 6.70 & 6.23 \\
$\mathrm{Glu}^{\mathrm{B} 13}$ & 8.94 & 8.87 & 8.66
\end{tabular}


Table S2. Crystallographic Unit-Cell Dimensions of Zinc Insulin Crystals

\begin{tabular}{|c|c|c|c|c|c|c|}
\hline $\begin{array}{l}\text { PDB } \\
\text { Code insulin }\end{array}$ & $\begin{array}{l}\text { space } \\
\text { group }\end{array}$ & unit cell & $\begin{array}{l}\text { hexamer } \\
\text { state }\end{array}$ & $\begin{array}{l}\text { asym } \\
\text { unit }\end{array}$ & $\begin{array}{l}\text { resolutic } \\
(\AA)\end{array}$ & ion references \\
\hline 1BEN HBD ${ }^{a}$ & R3 & $80.72,37.63$ & $\mathrm{~T}_{3} \mathrm{R}_{3}{ }^{\mathrm{f}}$ & 2 & 1.4 & Smith, D., 1996, Protein Sci. 1502 \\
\hline 1G7A CRY ${ }^{b}$ & $\mathrm{R} 3$ & $80.13,71.58$ & $\mathrm{~T}_{3} \mathrm{R}_{3}{ }^{\mathrm{f}}$ & 4 & 1.2 & Smith, D., .2001, Acta.Crystllogr.D57,1091 \\
\hline 1G7B CRY & $\mathrm{R} 3$ & $80.05,71.46$ & $\mathrm{~T}_{3} \mathrm{R}_{3}{ }^{\mathrm{f}}$ & 4 & 1.3 & Smith, D., 2001, Acta.Crystllogr.D57,1091 \\
\hline 1LPH IPH & R3 & $79.62,37.78$ & $\mathrm{~T}_{3} \mathrm{R}_{3}{ }^{\mathrm{f}}$ & 2 & 2.3 & Ciszak, E., 1995, Structure, 3: 615 \\
\hline 1MPJ IPH & R3 & $80.70,37.80$ & $\mathrm{~T}_{3} \mathrm{R}_{3}{ }^{\mathrm{f}}$ & 2 & 2.3 & Whittingham, J. L, 1995, Biochemistry,34: 15553 \\
\hline 1QJ0 & R3 & $80.94,37.63$ & $\mathrm{~T}_{3} \mathrm{R}_{3}{ }^{\mathrm{f}}$ & 2 & 2.4 & Tang, L., 1999, Biochemistry,38:12041 \\
\hline 1Q4V IPH & R3 & $80.49,37.94$ & $\mathrm{~T}_{3} \mathrm{R}_{3}{ }^{\mathrm{f}}$ & 2 & 2.0 & Wan, Z., 2003, Biochemistry, 44: 12770 \\
\hline 1TRZ & R3 & $80.64,37.78$ & $\mathrm{~T}_{3} \mathrm{R}_{3}{ }^{\mathrm{f}}$ & 2 & 1.6 & Ciszak, E. 1994, Biochemistry, 33: 1512 \\
\hline 1TYL TYL ${ }^{d}$ & $\mathrm{R} 3$ & $81.11,37.97$ & $\mathrm{~T}_{3} \mathrm{R}_{3}{ }^{\mathrm{f}}$ & 2 & 1.9 & Smith, D., 1994, ,Proc.Nat.Acad.Sci. 91:8851 \\
\hline 1TYM TYL & R3 & $80.88,37.60$ & $\mathrm{~T}_{3} \mathrm{R}_{3}{ }^{\mathrm{f}}$ & 2 & 1.9 & Smith, D., 1994, Proc.Nat.Acad.Sci. 91:8851 \\
\hline 1ZNI $4 \mathrm{INS}^{\mathrm{e}}$ & R3 & $80.70,37.60$ & $\mathrm{~T}_{3} \mathrm{R}_{3}{ }^{\mathrm{f}}$ & 2 & 1.5 & Bentley, G., 1976, Nature, 261:166 \\
\hline 1IZA & R3 & $80.60,37.60$ & $\mathrm{~T}_{3} \mathrm{R}_{3}{ }^{\mathrm{f}}$ & 2 & 2.5 & Bentley, G, .1992, JMB, 228:1163 \\
\hline 2TCI $\mathrm{SCN}^{\mathrm{f}}$ & R3 & $80.64,37.52$ & $\mathrm{~T}_{3} \mathrm{R}_{3}{ }^{\mathrm{f}}$ & 2 & 1.8 & Whittingham, J. L. 1995, Biochemistry, 34:1555 \\
\hline 3MTH MPB $^{\mathrm{g}}$ & R3 & $80.38,37.00$ & $\mathrm{~T}_{3} \mathrm{R}_{3}{ }^{\mathrm{f}}$ & 2 & 1.9 & Whittingham, J. L. 1995, Biochemistry, 34:155 \\
\hline 6INS & R3 & $79.22,37.75$ & $\mathrm{~T}_{3} \mathrm{R}_{3}$ & 2 & 2.0 & Derewenda, U. 1991, JMB, 220:425 \\
\hline
\end{tabular}

${ }^{\mathrm{a}}$ Chemical component, 4-hydroxybemzamide; ${ }^{\mathrm{b}}$ Chemical component, propane-1,2,3,-triol; ${ }^{\mathrm{c}}$ Chemical component, phenol; ${ }^{\mathrm{d}} \mathrm{Chemical}$ component, N-(4-hydroxyphenyl)acetamide (Tylenol); ${ }^{\mathrm{e}} 3$ zinc and 3 chloride ions; ${ }^{\mathrm{f}}$ Chemical component, thiocyanateion; ${ }^{\mathrm{g}}$ Chemical component, methylparaben.

\begin{tabular}{|c|c|c|c|c|c|c|c|}
\hline $\begin{array}{l}\text { PDB } \\
\text { Code }\end{array}$ & insulin & $\begin{array}{l}\text { space } \\
\text { group }\end{array}$ & unit cell & $\begin{array}{c}\text { hexamer } \\
\text { state }\end{array}$ & $\begin{array}{l}\text { asymm } \\
\text { unit }\end{array}$ & $\begin{array}{c}\text { resolutic } \\
(\AA)\end{array}$ & references \\
\hline $1 E V 3$ & $\mathrm{CRS}^{\mathrm{a}}$ & R3 & $78.9,39.5$ & $\mathrm{R}_{6}$ & 2 & 1.78 & Smith, D., 2000, Acta.Crystllogr. D56:1541 \\
\hline $1 E V 6$ & CRS & $\mathrm{P} 2_{1}$ & $61.3,61.7,47.5$ & $\mathrm{R}_{6}$ & 6 & 1.9 & Smith, D., 2000, Acta.Crystllogr. D56:1541 \\
\hline 1EVR & $\mathrm{RCO}^{\mathrm{b}}$ & R3 & $78.9,39.5$ & $\mathrm{R}_{6}$ & 2 & 1.9 & Smith, D. 2000, Acta.Crystllogr. D56:1541 \\
\hline 1QIY & $\mathrm{IPH}^{\mathrm{c}}$ & $\mathrm{P} 2_{1}$ & $61.1,62.1,48.3$ & $\mathrm{R}_{6}$ & 6 & $2.3 \mathrm{~T}$ & Tang, L.1999, Biochemistry, 38:1204 \\
\hline
\end{tabular}




\begin{tabular}{|c|c|c|c|c|c|c|c|}
\hline 1QIZ & $\mathrm{RCO}$ & $\mathrm{P} 2_{1}$ & $60.7,62.1,47.7$ & $\mathrm{R}_{6}$ & 6 & 2.0 & Tang, L.1999, Biochemistry, 38:1204 \\
\hline 1WAV & IPH & $\mathrm{P} 2_{1}$ & $49.2,60.9,48.2$ & $\mathrm{R}_{6}$ & 6 & 2.5 & Ding, J.1996, Sci.China, C39:144 \\
\hline 1XDA & IPH & R3 & $78.8,79.2$ & $\mathrm{R}_{6}$ & 4 & 1.8 & Whittingham, J. L. 1997, Biochemistry, 36:2826 \\
\hline 1ZEG & IPH & R3 & $77.7,39.2$ & $\mathrm{R}_{6}$ & 2 & 1.6 & Whittingham, J. L. 1998, Biochemistry, 37:11516 \\
\hline 1ZEH & CRS & R3 & $77.5,38.9$ & $\mathrm{R}_{6}$ & 2 & 1.5 & Whittingham, J. L. 1998, Biochemistry, 37:11516 \\
\hline 1ZEI & CRS & $\mathrm{P} 2_{1}$ & $54.0,64.8,48.9$ & $\mathrm{R}_{6}$ & 6 & 1.9 & Whittingham, J. L. 1998, Biochemistry, 37:11516 \\
\hline $1 Z \mathbf{N J}$ & IPH & $\mathrm{P} 2{ }_{1}$ & $61.2,61.6,48.1$ & $\mathrm{R}_{6}$ & 6 & 2.0 & PDB deposition \\
\hline
\end{tabular}

${ }^{\mathrm{a}}$ Chemical component, $m$-cresol ; ${ }^{\mathrm{b}} \mathrm{Chemical}$ component, resorcinol; ${ }^{\mathrm{c}} \mathrm{Chemical}$ component, phenol 
Table S3. Chemical-shift differences between ${ }^{1} \mathrm{H}$ NMR resonances of DKP-insulin and D-Ala ${ }^{\mathrm{B}}$ DKP-insulin (pH 7.0 and $\left.25^{\circ} \mathrm{C}\right)$

\begin{tabular}{lllll}
\hline residue & $\mathrm{NH}$ & $\mathrm{C}_{\alpha} \mathrm{H}$ & $\mathrm{C}_{\beta} \mathrm{H}$ & others
\end{tabular}

B7 Cys $\quad-0.47$

B9 Ser $\quad-0.18$

B12 Val $\quad-0.24$

Chemical shifts relative to TSP $(0 \mathrm{ppm})$. 
Table S4. DG/SA Restraints and Statistical Parameters

\begin{tabular}{lc} 
& $\mathrm{D}-\mathrm{Ala}^{\mathrm{B} 8}-\mathrm{DKP}$ \\
\hline NOE restraints: & 389 \\
total & 151 \\
sequential & 75 \\
medium & 119 \\
long-range & 56
\end{tabular}

Dihedral angle restraints: $\quad 42$

$\phi$-angles 27

$\chi_{1}$-angles 13

Hydrogen bonds: $\quad 36$

Main chain $\left(\mathrm{RMSD}^{\mathrm{a}}\right)$

$\begin{array}{ll}\alpha \text {-helices }^{\mathrm{b}} & 0.34 \\ \text { A chain } & 0.36 \\ \text { B chain } & 0.44\end{array}$

Side chain $\left(\mathrm{RMSD}^{\mathrm{a}}\right)$

$\begin{array}{ll}\alpha \text {-helices }^{\mathrm{b}} & 0.77 \\ \text { A chain } & 0.78 \\ \text { B chain } & 0.99\end{array}$

Average restraint violations
$\operatorname{NOE}(\AA)$
$0.046 \AA$
Dihedral angles
$0.35^{\circ}$

Deviations from ideal covalent geometry

$\begin{array}{ll}\text { bond length }(\AA) & 0.006 \AA \\ \text { bond angle } & 0.97^{\circ}\end{array}$

Empirical energy function ${ }^{\mathrm{c}}(\mathrm{kcal} / \mathrm{mole})$ 


$\begin{array}{lr}\text { NOE restraint energy } & 38.85 \pm 9.01 \\ \text { van der Waals } & 26.82 \pm 5.59 \\ \text { improper dihedral angles } & 9.52 \pm 1.02 \\ \text { constrainted dihedral angles } & 0.38 \pm 0.33 \\ \text { covalent bond lengths } & 30.92 \pm 1.48 \\ \text { bond angles } & 43.35 \pm 6.42\end{array}$

${ }^{\mathrm{a}} \alpha$-Helices span residues A2-A8, A12-A19, and B9-B19. ${ }^{\mathrm{b}} \mathrm{RMSD}$ values were calculated according to $\mathrm{C}_{\alpha}$ atoms of residues A2-A19 and B3-B24. ${ }^{\mathrm{c}} \mathrm{NOE}$ and dihedral force constants were $40 \mathrm{kcal} \AA^{-2}$ and $40 \mathrm{kcal} \mathrm{radian}^{-2}$, respectively $(1 \mathrm{kcal}=4.18 \mathrm{~kJ})$. Only chi-1 related intraresidue NOEs were counted for D-Ala ${ }^{\mathrm{B} 8}$-DKP-insulin. 
Table S5. Table of phi-psi angles of B8 residues

\begin{tabular}{|c|c|c|}
\hline & \multicolumn{2}{|c|}{ Dihedral angle (deg.) } \\
\hline & $\varphi$ & $\psi$ \\
\hline insulin T-state B8 Gly $^{\mathrm{a}}$ & $56.4 \pm 4.1$ & $-134.6 \pm 4.0$ \\
\hline insulin R-state B8 $\mathrm{Gly}^{b}$ & $-63.0 \pm 3.2$ & $-34.2 \pm 5.16$ \\
\hline B8-D-Ala-DKP B8 D-Ala & $22.1 \pm 5.2$ & $-103.5 \pm 4.6$ \\
\hline B8-D-Ala-DKP B14 D-Ala & $-67.7 \pm 3.6$ & $-39.0 \pm 3.0$ \\
\hline
\end{tabular}

${ }^{a}$ Dihedral angles were calculated based on 9 crystallographic protomers obtained from PDB structures 4INS, 1TYL, 1PID, 1APH, 1TRZ, 1TYL, 1TYM and 1ZNI. ${ }^{b}$ Dihedral angles were likewise calculated based on PDB structures 1TYL, 1TRZ, 1EV6, 1EV3, $1 \mathrm{ZNJ}$, and 6INS.

4INS: Baker EN, Blundell TL, Cutfield JF, Cutfield SM, Dodson EJ, Dodson GG, Hodgkin DM, Hubbard RE, Isaacs NW, Reynolds CD, et al. (1988) The structure of 2Zn pig insulin crystals at 1.5 A resolution. Philos Trans $R$ Soc Lond B Biol Sci. 319:369-456.

1ZNI: Bentley G, Dodson E, Dodson G, Hodgkin D, Mercola D. (1976) Structure of insulin in 4-zinc insulin.

Nature. 261:166-8.

1ZNJ : M. G. W. Turkenburg, J. L. Whittingham, J. P. Turkenburg, G. G. Dodson, U.

Derewenda, G. D. Smith, E. J. Dodson, Z. S. Derewenda, B. Xiao (1998?) Structure determination and refinement of two crystal forms of native insulins (to be published).

1TYL: Smith GD, Ciszak E. (1994) The structure of a complex of hexameric insulin and 4'hydroxyacetanilide.

Proc Natl Acad Sci U S A. 91:8851-5.

1TRZ: Ciszak E, Smith GD. (1994) Crystallographic evidence for dual coordination around zinc in the T3R3 human insulin hexamer. Biochemistry. 33:1512-7.

1PID: Brange J, Dodson GG, Edwards DJ, Holden PH, Whittingham JL. (1997) A model of insulin fibrils derived from the $\mathrm{x}$-ray crystal structure of a monomeric insulin (despentapeptide insulin). Proteins. 27:507-16.

1APH: Gursky O, Badger J, Li Y, Caspar DL. (1992) Conformational changes in cubic insulin crystals in the $\mathrm{pH}$ range 7-11. Biophys J. 63:1210-20.

1TYM ( $\mathrm{T}_{3} \mathrm{R}_{3}$ hexamer): Smith GD, Ciszak E. (1994) The structure of a complex of hexameric insulin and 4'-hydroxyacetanilide. Proc Natl Acad Sci U S A. 91:8851-5.

1EV3: Smith GD, Ciszak E, Magrum LA, Pangborn WA, Blessing RH. (2000) R6 hexameric insulin complexed with m-cresol or resorcinol. Acta Crystallogr D Biol Crystallogr. 56:1541-8. 
1EV6: Smith GD, Ciszak E, Magrum LA, Pangborn WA, Blessing RH. (2000) R6 hexameric insulin complexed with m-cresol or resorcinol. (2000) Acta Crystallogr D Biol Crystallogr. 56:1541-8.

6INS: Derewenda U, Derewenda Z, Dodson EJ, Dodson GG, Bing X, Markussen J. (1991) Xray analysis of the single chain B29-A1 peptide-linked insulin molecule. A completely inactive analogue. J Mol Biol. 220:425-33. 
Table S6. Chemical shifts of the assigned ${ }^{1} \mathrm{H}$ NMR resonances of D-Ala ${ }^{\mathrm{B}}-\mathrm{DKP}\left(\mathrm{pH} 7.0\right.$ and $\left.25^{\circ} \mathrm{C}\right)$

\begin{tabular}{|c|c|c|c|c|}
\hline residue & $\mathrm{NH}$ & $\mathrm{C}_{\alpha} \mathrm{H}$ & $\mathrm{C}_{\beta} \mathrm{H}$ & Others \\
\hline \multicolumn{5}{|l|}{ A1 Gly } \\
\hline A2 Ile & & 3.92 & 1.12 & $\mathrm{C}_{\gamma} \mathrm{H}_{2} 1.02,0.82, \mathrm{C}^{\prime} \mathrm{H}_{3} 0.67, \mathrm{C}_{\delta} \mathrm{H}_{3} 0.47$ \\
\hline A3 Val & 8.28 & 3.60 & 1.97 & $\mathrm{C} \gamma \mathrm{H}_{3} 0.98,0.92$ \\
\hline A4 Glu & 8.38 & 4.16 & \multicolumn{2}{|c|}{$2.19,2.06 \mathrm{C} \gamma \mathrm{H}_{2} 2.52,2.30$} \\
\hline A5 Gln & 8.28 & 4.15 & \multicolumn{2}{|c|}{$2.16,2.03 \mathrm{C} \gamma \mathrm{H}_{2} 2.54,2.42$} \\
\hline $\begin{array}{l}\text { A6 Cys } \\
\text { A7 Cys }\end{array}$ & $\begin{array}{l}8.35 \\
8.28\end{array}$ & $\begin{array}{l}5.01 \\
4.91\end{array}$ & \multicolumn{2}{|c|}{$\begin{array}{l}2.87,2.87 \\
3.85,3.35\end{array}$} \\
\hline A8 $\mathrm{Thr}$ & 8.29 & 4.07 & 4.46 & \multirow[t]{2}{*}{$\mathrm{C}_{\gamma} \mathrm{H}_{3} 1.28$} \\
\hline A9 Ser & 8.10 & 4.79 & 4.01 & \\
\hline A10 lle & 7.84 & 4.34 & 1.57 & \multirow[t]{2}{*}{$\mathrm{C}_{\gamma} \mathrm{H}_{2} 1.09,0.39, \mathrm{C}_{\gamma^{\prime}} \mathrm{H} 30.68, \mathrm{C}_{\delta} \mathrm{H} 30.50$} \\
\hline $\begin{array}{l}\text { A11 Cys } \\
\text { A12 Ser } \\
\text { A13 leu }\end{array}$ & $\begin{array}{l}9.90 \\
8.75 \\
8.75\end{array}$ & $\begin{array}{l}4.95 \\
4.67 \\
3.90\end{array}$ & $\begin{array}{l}3.21 \\
4.21 \\
1.57\end{array}$ & \\
\hline $\begin{array}{l}\text { A14 Tyr } \\
\text { A15 Gln }\end{array}$ & $\begin{array}{l}7.67 \\
7.50\end{array}$ & $\begin{array}{l}4.34 \\
4.00\end{array}$ & \multicolumn{2}{|c|}{$\begin{array}{l}\text { 3.03,3.03 C2,6H 7.14,C3,5H } 6.89 \\
2.43,2.37 \mathrm{C} \gamma \mathrm{H} 22.27,2.05, \mathrm{~N}_{\delta} \mathrm{H}_{2} 7.56,7.03\end{array}$} \\
\hline A16 Leu & 7.88 & 4.16 & \multicolumn{2}{|c|}{$2.00,1.45 \mathrm{C} \gamma \mathrm{H} 1.77, \mathrm{C}_{\delta} \mathrm{H} 30.79,0.74$} \\
\hline A17 Glu & 8.06 & 4.22 & \multicolumn{2}{|c|}{$2.11,2.05 \mathrm{C} \gamma \mathrm{H}_{2} 2.40,2.26$} \\
\hline A18 Asn & 7.35 & 4.50 & \multicolumn{2}{|c|}{$2.68,2.57 \mathrm{~N} \delta \mathrm{H}_{2} 7.21,6.63$} \\
\hline $\begin{array}{l}\text { A19 Tyr } \\
\text { A20 Cys } \\
\text { A21 Asn }\end{array}$ & $\begin{array}{l}8.00 \\
7.36 \\
8.08\end{array}$ & $\begin{array}{l}4.34 \\
5.15 \\
4.51\end{array}$ & $\begin{array}{l}3.44 \\
3.33 \\
2.80\end{array}$ & $\begin{array}{l}38, C 3,5 \text { H } 6.82 \\
49,6.42\end{array}$ \\
\hline $\begin{array}{l}\text { B1 Phe } \\
\text { B2 Val }\end{array}$ & 8.28 & $\begin{array}{l}4.28 \\
4.08\end{array}$ & \multicolumn{2}{|c|}{$3.16,3.16 \mathrm{C} 2,6 \mathrm{H} 7.22, \mathrm{C} 3,5 \mathrm{H} 7.33$} \\
\hline $\begin{array}{l}\text { B3 Asn } \\
\text { B4 Gln }\end{array}$ & $\begin{array}{l}8.56 \\
8.20\end{array}$ & $\begin{array}{l}4.66 \\
4.52\end{array}$ & \multicolumn{2}{|c|}{$2.85,2.75$} \\
\hline $\begin{array}{l}\text { B5 His } \\
\text { B6 Leu }\end{array}$ & $\begin{array}{l}8.55 \\
9.09\end{array}$ & $\begin{array}{l}4.42 \\
4.61\end{array}$ & \multicolumn{2}{|c|}{$3.53,3.18 \mathrm{C} 2 \mathrm{H} 8.35, \mathrm{C} 4 \mathrm{H} 7.27$} \\
\hline $\begin{array}{l}\text { B7 Cys } \\
\text { B8 Ala }\end{array}$ & $\begin{array}{l}8.80 \\
9.28\end{array}$ & $\begin{array}{l}5.00 \\
4.27\end{array}$ & \multicolumn{2}{|c|}{$3.24,2.99$} \\
\hline $\begin{array}{l}\text { B9 Ser } \\
\text { B10 Asp } \\
\text { B11 Leu }\end{array}$ & $\begin{array}{l}9.15 \\
7.94 \\
6.87\end{array}$ & $\begin{array}{l}4.22 \\
4.45 \\
3.93\end{array}$ & \multicolumn{2}{|c|}{$\begin{array}{l}4.12,4.02 \\
3.04,2.68 \\
1.93,1.11 \mathrm{C} \gamma \mathrm{H} 1.25, \mathrm{C}_{\delta} \mathrm{H} 3 \quad 0.75,0.68\end{array}$} \\
\hline B12 Val & 7.20 & 3.22 & \multicolumn{2}{|c|}{$2.12 \quad{\mathrm{C} \gamma \mathrm{H}_{3}}^{1.03,0.98}$} \\
\hline B13 Glu & 7.99 & 4.11 & \multicolumn{2}{|c|}{$2.37,2.13 \mathrm{C} \gamma \mathrm{H}_{2} 2.50$} \\
\hline B14 Ala & 7.60 & 4.11 & \multicolumn{2}{|c|}{$\mathrm{C}_{\beta} \mathrm{H} 31.40$} \\
\hline B15 Leu & 8.07 & 3.74 & \multicolumn{2}{|c|}{$1.02,0.98 \mathrm{C} \gamma \mathrm{H} 1.30, \mathrm{C}_{\delta} \mathrm{H} 30.56,0.20$} \\
\hline $\begin{array}{l}\text { B16 Tyr } \\
\text { B17 Leu }\end{array}$ & $\begin{array}{l}8.19 \\
7.70\end{array}$ & $\begin{array}{l}4.42 \\
4.11\end{array}$ & \multicolumn{2}{|c|}{$\begin{array}{l}3.20,3.20 \mathrm{C} 2,6 \mathrm{H} 7.30, \mathrm{C} 3,5 \mathrm{H} 6.87 \\
1.95,1.78 \mathrm{C} \gamma \mathrm{H} 1.80, \mathrm{C}_{\delta} \mathrm{H} 30.99,0.85\end{array}$} \\
\hline B18 Val & 8.45 & 3.79 & \multicolumn{2}{|c|}{$2.00 \quad \mathrm{C}_{\gamma} \mathrm{H}_{3} 1.03,0.89$} \\
\hline $\begin{array}{l}\text { B19 Cys } \\
\text { B20 Gly }\end{array}$ & $\begin{array}{l}8.83 \\
7.80\end{array}$ & $\begin{array}{l}4.88 \\
4.01,3.90\end{array}$ & \multicolumn{2}{|c|}{$3.32,2.95$} \\
\hline B21 Glu & 9.15 & 4.22 & \multicolumn{2}{|c|}{$2.23,2.10 \mathrm{C} \gamma \mathrm{H}_{2} 2.40$} \\
\hline B22 Arg & 8.14 & 4.21 & \multicolumn{2}{|c|}{ 2.20,2.10 $\mathrm{C}_{\gamma} \mathrm{H}_{2}$ 1.90,1.85, $\mathrm{C}_{\delta} \mathrm{H}_{2} 3.40,3.35, \mathrm{~N}_{\varepsilon} \mathrm{H}_{2} 7.27$} \\
\hline $\begin{array}{l}\text { B23 Gly } \\
\text { B24 Phe } \\
\text { B25 Phe } \\
\text { B26 Tyr } \\
\text { B27 Thr }\end{array}$ & $\begin{array}{l}7.37 \\
7.60 \\
8.64 \\
8.18 \\
7.70\end{array}$ & $\begin{array}{l}4.15,3.83 \\
5.20 \\
4.91 \\
4.62 \\
4.40\end{array}$ & $\begin{array}{l}3.11 \\
3.22 \\
3.00 \\
4.10\end{array}$ & $\begin{array}{l}78, \mathrm{C} 3,5 \mathrm{H} 6.90 \\
\mathrm{C} 2,6 \mathrm{H} 7.25, \mathrm{C} 3,5 \mathrm{H} 7.36 \\
98, \mathrm{C} 3,5 \mathrm{H} 6.69 \\
\mathrm{C}^{2} \mathrm{H}_{3} 1.19\end{array}$ \\
\hline B28 Lys & 8.29 & 4.36 & 1.82 & $5, \mathrm{C}_{\delta} \mathrm{H} 21.65$ \\
\hline & & & & $\mathrm{C}_{\varepsilon} \mathrm{H}_{2} 3.02, \mathrm{~N}_{\varepsilon} \mathrm{H} 27.27$ \\
\hline B29 Pro & & 4.48 & 2.32 & $0, \mathrm{C} \delta \mathrm{H}_{2} 3.77,3.56$ \\
\hline B30 Thr & 7.83 & 4.16 & 4.26 & $\mathrm{C}_{\gamma} \mathrm{H}_{3} 1.21$ \\
\hline
\end{tabular}


Table S7. Chemical shifts of assigned ${ }^{1} \mathrm{H}$ NMR resonances of D-Ala ${ }^{\mathrm{B} 8}$-DKP-human insulin $\left(20 \% \mathrm{dAA}\right.$ and $\left.25^{\circ} \mathrm{C}\right)$

\begin{tabular}{|c|c|c|c|c|}
\hline residue & $\mathrm{NH}$ & $\mathrm{C} \alpha \mathrm{H}$ & $\mathrm{C} \beta \mathrm{H}$ & others \\
\hline A1 Gly & & $4.05,4.05$ & & \\
\hline A2 Ile & 8.55 & 3.97 & 1.22 & $\mathrm{C}_{\gamma} \mathrm{H}_{2} 0.97, \mathrm{C}_{\gamma^{\prime}} \mathrm{H}_{3} 0.79, \mathrm{C}_{\delta} \mathrm{H}_{3} 0.65$ \\
\hline A3 Val & 8.13 & 3.65 & 2.00 & $\mathrm{C}_{\gamma} \mathrm{H}_{3} 0.96,0.91$ \\
\hline A4 Glu & 8.28 & 4.10 & $2.15,2.10$ & $\mathrm{C}_{\gamma} \mathrm{H}_{2} 2.52,2.44$ \\
\hline A5 Gln & & 4.23 & $2.15,2.07$ & $\mathrm{C}_{\gamma} \mathrm{H}_{2} 2.60,2.38$ \\
\hline A6 Cys & 8.32 & 4.96 & $3.38,2.90$ & \\
\hline A7 Cys & 8.28 & 4.89 & $3.85,3.32$ & \\
\hline A8 $\mathrm{Thr}$ & 8.29 & 4.08 & 4.42 & $\mathrm{C}_{\gamma} \mathrm{H}_{3} 1.28$ \\
\hline A9 Ser & 7.43 & 4.82 & $4.07,3.93$ & \\
\hline \multirow[t]{2}{*}{ A10 Ile } & 7.86 & 4.42 & 1.57 & $\mathrm{C}_{\gamma} \mathrm{H}_{2} 1.10,0.45$ \\
\hline & & & & $\mathrm{C}_{\gamma^{\prime} \mathrm{H}_{3}} 0.67, \mathrm{C}_{\delta} \mathrm{H}_{3} 0.54$ \\
\hline A11 Cys & 9.79 & & & \\
\hline A12 Ser & 8.78 & 4.65 & $4.35,4.04$ & \\
\hline A13 Leu & 8.68 & 3.89 & $1.38,1.38$ & $\mathrm{C}_{\gamma} \mathrm{H} 1.45, \mathrm{C}_{\delta} \mathrm{H}_{3} \quad 0.82,0.77$ \\
\hline A14 Tyr & 7.52 & 4.19 & $3.02,2.94$ & $\mathrm{C} 2,6 \mathrm{H} 7.08, \mathrm{C} 3,5 \mathrm{H} 6.86$ \\
\hline A15 Gln & 7.59 & 4.01 & $2.41,2.06$ & $\begin{array}{l}\mathrm{C}_{\gamma} \mathrm{H}_{2} 2.48,2.48 \\
\mathrm{~N}_{\delta} \mathrm{H}_{2}\end{array}$ \\
\hline A16 Leu & 8.11 & 4.19 & $1.97,1.62$ & $\mathrm{C}_{\gamma} \mathrm{H} 1.77, \mathrm{C}_{\delta} \mathrm{H}_{3} 0.89,0.82$ \\
\hline A17 Glu & 8.12 & 4.28 & $2.14,2.14$ & $\mathrm{C}_{\gamma} \mathrm{H}_{2} 2.53$ \\
\hline A18 Asn & 7.45 & 4.50 & $2.65,2.57$ & $\mathrm{~N}_{\delta} \mathrm{H}_{2} 7.19,6.57$ \\
\hline A19 Tyr & 7.94 & 4.48 & $3.39,3.00$ & $\mathrm{C} 2,6 \mathrm{H} 7.36, \mathrm{C} 3,5 \mathrm{H} 6.80$ \\
\hline $\begin{array}{l}\text { A20 Cys } \\
\text { A21 Asn }\end{array}$ & $\begin{array}{l}7.43 \\
8.29\end{array}$ & $\begin{array}{l}4.41 \\
4.77\end{array}$ & $\begin{array}{l}3.27,2.86 \\
2.91,2.79\end{array}$ & $\mathrm{~N}_{\delta} \mathrm{H}_{2}$ \\
\hline $\begin{array}{l}\text { A21 Asn } \\
\text { B1 Phe }\end{array}$ & & 4.30 & $\begin{array}{l}2.91,2.39 \\
3.21,3.21\end{array}$ & $\begin{array}{l}{ }_{\delta}{ }^{\mathrm{H}_{2}} \\
\mathrm{C} 2,6 \mathrm{H} 7.26,7.38 \quad, \mathrm{C} 3,5 \mathrm{H}\end{array}$ \\
\hline B2 Val & 8.20 & 4.17 & 1.94 & $\mathrm{C}_{\gamma} \mathrm{H}_{3} 0.89$ \\
\hline B3 Asn & 8.50 & 4.77 & 2.77 & $\mathrm{~N}_{\delta} \mathrm{H}_{2}$ \\
\hline B4 Gln & 8.42 & 4.52 & $2.12,1.94$ & $\mathrm{C}_{\gamma} \mathrm{H}_{2} 2.28,2.21$ \\
\hline B5 His & 8.64 & 4.51 & $3.60,3.29$ & $\mathrm{C} 2 \mathrm{H} 8.63, \mathrm{C} 4 \mathrm{H} 7.44$ \\
\hline B6 Leu & 9.02 & 4.61 & $1.78,0.86$ & $\mathrm{C}_{\gamma} \mathrm{H} 1.63, \mathrm{C}_{\delta} \mathrm{H}_{3} 0.89,0.88$ \\
\hline B7 Cys & 8.31 & 5.00 & $3.23,2.97$ & \\
\hline B8 Ala & 9.05 & 4.25 & $\mathrm{C}_{\beta} \mathrm{H}_{3} 1.45$ & \\
\hline B9 Ser & 9.05 & 4.12 & 4.00 & \\
\hline B10 Asp & 8.17 & 4.55 & $3.23,2.85$ & \\
\hline B11 Leu & 7.03 & 4.00 & $1.98,1.20$ & $\mathrm{C}_{\gamma} \mathrm{H} 1.33, \mathrm{C}_{\delta} \mathrm{H}_{3} 0.84,0.76$ \\
\hline B12 Val & 7.38 & 3.34 & 2.10 & $\mathrm{C}_{\gamma} \mathrm{H}_{3} 0.96$ \\
\hline B13 Glu & 8.05 & 4.15 & $2.22,2.11$ & $\mathrm{C}_{\gamma} \mathrm{H}_{2} 2.57$ \\
\hline B14 Ala & 7.81 & 4.10 & $\mathrm{C}_{\beta} \mathrm{H}_{3} 1.51$ & \\
\hline B15 Leu & 8.11 & 3.90 & $1.39,1.39$ & $\mathrm{C}_{\gamma} \mathrm{H} 1.51, \mathrm{C}_{\delta} \mathrm{H}_{3} 0.69,0.50$ \\
\hline $\begin{array}{l}\text { B16 Tyr } \\
\text { B17 Leu }\end{array}$ & $\begin{array}{l}8.19 \\
7.97\end{array}$ & $\begin{array}{l}4.28 \\
4.10\end{array}$ & $\begin{array}{l}3.18 \\
1.98,1.66\end{array}$ & $\begin{array}{l}\mathrm{C} 2,6 \mathrm{H} 7.14, \mathrm{C} 3,5 \mathrm{H} 6.81 \\
\mathrm{C}_{\gamma} \mathrm{H} 1.89, \mathrm{C}_{\delta} \mathrm{H}_{3} 0.95\end{array}$ \\
\hline B18 Val & 8.62 & 3.89 & 2.12 & $\mathrm{C}_{\gamma} \mathrm{H}_{3} 1.05,0.90$ \\
\hline B19 Cys & 8.78 & 4.81 & $3.28,2.97$ & \\
\hline B20 Gly & 7.77 & 3.97 & & \\
\hline B21 Glu & 8.45 & 4.25 & $2.21,2.12$ & $\mathrm{C}_{\gamma} \mathrm{H}_{2} 2.54$ \\
\hline B22 Arg & 8.02 & 4.24 & $2.01,1.98$ & $\mathrm{C}_{\gamma} \mathrm{H}_{2} 1.81,1.78, \mathrm{C}_{\delta} \mathrm{H}_{2} 3.29$ \\
\hline B23 Gly & 7.73 & $4.04,3.87$ & & \\
\hline B24 Phe & 7.75 & 4.85 & $3.14,2.93$ & $\mathrm{C} 2,6 \mathrm{H} 6.93, \mathrm{C} 3,5 \mathrm{H} 7.07$ \\
\hline B25 Phe & 8.29 & 4.69 & $3.13,3.05$ & $\mathrm{C} 2,6 \mathrm{H} 7.02, \mathrm{C} 3,5 \mathrm{H} 7.30$ \\
\hline B26 Tyr & 8.01 & 4.67 & $3.00,2.97$ & $\mathrm{C} 2,6 \mathrm{H} 7.04, \mathrm{C} 3,5 \mathrm{H} 6.78$ \\
\hline B27 Thr & 7.83 & 4.35 & 4.11 & $\mathrm{C}_{\gamma} \mathrm{H}_{3} 1.18$ \\
\hline \multirow[t]{2}{*}{ B28 Lys } & 8.19 & 4.51 & $1.84,1.75$ & $\mathrm{C}_{\gamma} \mathrm{H}_{2}{ }_{1.50}, \mathrm{C}_{\delta} \mathrm{H}_{2} 1.62$ \\
\hline & & & & $\mathrm{C}_{\varepsilon} \mathrm{H}_{2} \quad 3.02, \mathrm{~N}_{\varepsilon} \mathrm{H}_{2}$ \\
\hline \multirow[t]{2}{*}{ B29 Pro } & & 4.55 & $2.30,2.08$ & $\mathrm{C}_{\gamma} \mathrm{H}_{2} 2.00$ \\
\hline & & & & $\mathrm{C}_{\delta}^{\prime} \mathrm{H}_{2} 3.83,3.67$ \\
\hline B30 Thr & 8.11 & 4.42 & 4.47 & $\mathrm{C}_{\gamma} \mathrm{H}_{3} 1.23$ \\
\hline
\end{tabular}

${ }^{\mathrm{a}}$ Chemical-shifts are relative to 3,3,3-Trimethylsilylpropionate ( $\left.0 \mathrm{ppm}\right)$. 
Table S8. Restraint list.

!BIOSYM restraint 1

!

\#remote prochiral center

\begin{tabular}{|c|c|c|c|c|}
\hline 1:LEU_32 $\overline{2}^{2} \mathrm{HD} 1$ * & 1:LEU 32:HD2* & 1:LEU_32:CD1 & 1:LEU_32:CD2 & $1:$ LEU_ $32:$ CG \\
\hline $1: \mathrm{VAL}^{-} 3: \mathrm{HGI}^{*}$ & $1: \mathrm{VAL}^{-} 3: \mathrm{HG} 2$ * & $1: \mathrm{VAL}^{-} 3: \mathrm{CG1}$ & $1: \mathrm{VAL}^{-}{ }^{-} 3: \mathrm{CG} 2$ & 1:VAL_3: $\mathrm{CB}$ \\
\hline 1:LEU-27 :HD1* & $1:$ LEU_27 : HD2* & 1:LEU $27: C D 1$ & 1:LEU_ 27:CD2 & $1: \mathrm{LEU}^{-} 27: \mathrm{CG}$ \\
\hline 1:VAL_39:HGI* & $1: \mathrm{VAL}^{-} 39: \mathrm{HG} 2$ * & $1: \mathrm{VAL}^{-} 39: \mathrm{CG1}$ & $1: \mathrm{VAL}^{-} 39: \mathrm{CG} 2$ & $1: \mathrm{VAL}^{-} 39: \mathrm{CB}$ \\
\hline 1:LEU_-16:HD1* & $1: \mathrm{LEU}^{-} 16: \mathrm{HD} 2$ * & 1:LEU $16: \mathrm{CD} 1$ & 1:LEU-16:CD2 & $1: \mathrm{LEU}^{-} 16: \mathrm{CG}$ \\
\hline :LEU_36:HDI* & $1: \mathrm{LEU}_{-} 36: \mathrm{HD} 2$ * & 1:LEU_36:CD1 & 1:LEU_36:CD2 & $1: \mathrm{LEU}^{-} 36: \mathrm{CG}$ \\
\hline : LEU $13: \mathrm{HDI}^{-}$* & $1: \mathrm{LEU}^{-1} 13: \mathrm{HD} 2$ * & $1: \mathrm{LEU}^{-} 13: \mathrm{CD} 1$ & 1:LEU-13:CD2 & $1: \mathrm{LEU}^{-} 13: \mathrm{CG}$ \\
\hline :VAL_23:HGI* & $1: \mathrm{VAL}^{-} 23: \mathrm{HG}^{2}$ * & 1:VAL_23:CG1 & 1:VAL_23:CG2 & $1: \mathrm{VAL}^{-} 23: \mathrm{CB}$ \\
\hline :VAL_33:HGI* & $1: \mathrm{VAL}^{-} 33: \mathrm{HG} 2$ * & $1: \mathrm{VAL}^{-} 33: \mathrm{CG1}$ & $1: \mathrm{VAL}^{-} 33: \mathrm{CG} 2$ & $1: \mathrm{VAL}^{-} 3$ \\
\hline
\end{tabular}

\#chiral

1:ILE 2:CA S

1:VAL $3: \mathrm{CA} \quad \mathrm{S}$

1:GLU- $4: \mathrm{CA} \quad \mathrm{S}$

1:GLN_- $5: C A \quad S$

1:CYS $6: \mathrm{CA} \quad \mathrm{R}$

1: $\mathrm{CYS}^{-}$- $: \mathrm{CA} \quad \mathrm{R}$

1:THR $8: \mathrm{CA} \quad \mathrm{S}$

1:SER_9:CA S

1: ILE $^{-} 10: \mathrm{CA} \quad \mathrm{S}$

1:CYS_11:CA R

1:SER_12:CA S

1:LEU $13: \mathrm{CA} \quad \mathrm{S}$

1:TYR_14:CA S

1:GLN_15:CA S

1:LEU_16:CA S

1:GLU- $17: \mathrm{CA} \quad \mathrm{S}$

1:ASN_18:CA S

1:TYR_19:CA S

1:CYS_20:CA R

1:ASNC_ $21: \mathrm{CA} \quad \mathrm{S}$

1:PHEN_22:CA S

1:VAL_23:CA S

1:ASN_24:CA S

1:GLN_25:CA S

1:HIS_26:CA S

1:LEU_27:CA S

1:CYS_28:CA R

1:ALA_29:CA R

1:SER_30:CA S

$1: \mathrm{ASP}_{-} 31: \mathrm{CA} \quad \mathrm{S}$

1:LEU_ $32: C A \quad S$

1:VAL_33:CA S

1:GLU- $34: C A \quad S$

1:ALA_35:CA S

1:LEU_36:CA S

1:TYR_37:CA S

1:LEU_38:CA S

1:VAL_39:CA S

1: $\mathrm{CYS}^{-} 40: \mathrm{CA} \quad \mathrm{R}$

1:GLU- $42: \mathrm{CA} \quad \mathrm{S}$

$1: \mathrm{ARG}+{ }^{-} 43: \mathrm{CA} \quad \mathrm{S}$

$1:$ PHE_ $\overline{4} 5: \mathrm{CA} \quad \mathrm{S}$ 


\begin{tabular}{|c|c|}
\hline 1:PHE $46: \mathrm{CA}$ & S \\
\hline 1:TYR_47:CA & S \\
\hline 1:THR $48: \mathrm{CA}$ & S \\
\hline 1:LYST+49:CA & S \\
\hline $1: \mathrm{PRO} \_\overline{5} 0: \mathrm{CA}$ & $\mathrm{S}$ \\
\hline 1:ILE²:CB & $\mathrm{R}$ \\
\hline 1:ILE- $10: \mathrm{CB}$ & $\mathrm{R}$ \\
\hline $1:$ THR_8:CB & $\mathrm{R}$ \\
\hline $1: \mathrm{THR}^{-} 48: \mathrm{CB}$ & $\mathrm{R}$ \\
\hline$! \quad-$ & \\
\hline \#distance & \\
\hline 1:GLYN_1:CA & $1: \mathrm{VAL} 3: \mathrm{CA}$ \\
\hline 1:ILE_ $\overline{2}: \mathrm{CA}$ & $1:$ GLU-_ $4: \mathrm{CA}$ \\
\hline $1: \mathrm{VAL}^{-} 3: \mathrm{CA}$ & $1: G L N \quad \overline{5}: C A$ \\
\hline $1: \mathrm{GLU}-4$ - $\mathrm{CA}$ & $1: \mathrm{CYS}^{-} 6: \mathrm{CA}$ \\
\hline $1:$ GLN $\overline{5}: \mathrm{CA}$ & $1: \mathrm{CYS}^{-} 7: \mathrm{CA}$ \\
\hline $1: \mathrm{CYS}^{-} 6: \mathrm{CA}$ & $1:$ THR_ $8: \mathrm{CA}$ \\
\hline $1: \mathrm{CYS}^{-} 7: \mathrm{CA}$ & 1:SER $9: \mathrm{CA}$ \\
\hline 1:THR_8:CA & 1:ILE_10:CA \\
\hline 1:SER ${ }^{-} 9: \mathrm{CA}$ & $1: \mathrm{CYS}^{-} 11: \mathrm{CA}$ \\
\hline 1:ILE_10:CA & $1: \mathrm{SER}^{-} 12: \mathrm{CA}$ \\
\hline 1: $\mathrm{CYS}^{-1} 11: \mathrm{CA}$ & $1: \mathrm{LEU}^{-} 13: \mathrm{CA}$ \\
\hline 1:SER_12:CA & $1: \mathrm{TYR}^{-} 14: \mathrm{CA}$ \\
\hline 1: LEU $13: \mathrm{CA}$ & $1: \mathrm{GLN}^{-} 15: \mathrm{CA}$ \\
\hline 1:TYR_14:CA & $1:$ LEU_16:CA \\
\hline $1: \mathrm{GLN}^{-} 15: \mathrm{CA}$ & $1: \mathrm{GLU}=17: \mathrm{CA}$ \\
\hline 1:LEU_16:CA & $1: \mathrm{ASN}=\overline{1} 8: \mathrm{CA}$ \\
\hline 1:GLU- $17: \mathrm{CA}$ & 1:TYR_19:CA \\
\hline $1: \mathrm{ASN} \_\overline{1} 8: \mathrm{CA}$ & $1: \mathrm{CYS}^{-} 20: \mathrm{CA}$ \\
\hline 1:TYR_19:CA & $1:$ ASNC $21: \mathrm{CA}$ \\
\hline 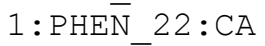 & $1: \mathrm{ASN} \quad \overline{2} 4: \mathrm{CA}$ \\
\hline $1: \mathrm{VAL}=\overline{2} 3: \mathrm{CA}$ & $1: \mathrm{GLN}^{-} 25: \mathrm{CA}$ \\
\hline 1:ASN_24:CA & $1: \mathrm{HIS}^{-} 26: \mathrm{CA}$ \\
\hline 1:GLN_25:CA & 1:LEU_27:CA \\
\hline 1:HIS_- $26: \mathrm{CA}$ & $1: \mathrm{CYS}^{-} 28: \mathrm{CA}$ \\
\hline 1:LEU_27:CA & $1:$ ALA_29:CA \\
\hline 1:CYS_28:CA & $1: S E R \_30: C A$ \\
\hline 1:ALA_29:CA & $1: \mathrm{ASP}=31: \mathrm{CA}$ \\
\hline 1:SER_30:CA & $1:$ LEU_ $32: \mathrm{CA}$ \\
\hline $1: \mathrm{ASP}=31: \mathrm{CA}$ & $1: \mathrm{VAL}^{-} 33: \mathrm{CA}$ \\
\hline $1:$ LEU_ $32: \mathrm{CA}$ & $1: \mathrm{GLU}-\_34: \mathrm{CA}$ \\
\hline $1: \mathrm{VAL}^{-} 33: \mathrm{CA}$ & $1:$ ALA_ $35: C A$ \\
\hline $1:$ GLU-_34:CA & $1: \mathrm{LEU}^{-} 36: \mathrm{CA}$ \\
\hline 1:ALA_ $35: \mathrm{CA}$ & $1:$ TYR_37:CA \\
\hline 1:LEU_36:CA & $1:$ LEU_38:CA \\
\hline 1:TYR_37:CA & $1: \mathrm{VAL}^{-} 39: \mathrm{CA}$ \\
\hline 1:LEU_38:CA & $1: \mathrm{CYS}^{-} 40: \mathrm{CA}$ \\
\hline $1: V^{2} \_39: \mathrm{CA}$ & $1:$ GLY_ $41: \mathrm{CA}$ \\
\hline $1: \mathrm{CYS}^{-} 40: \mathrm{CA}$ & $1:$ GLU-_ $42: \mathrm{CA}$ \\
\hline $1:$ GLY_41:CA & $1: \mathrm{ARG}+\_43: \mathrm{CA}$ \\
\hline $1: \mathrm{GLU}-42: \mathrm{CA}$ & $1:$ GLY $\overline{4} 4:$ CA \\
\hline $1: \mathrm{ARG}+{ }^{-} 43: \mathrm{CA}$ & $1: \mathrm{PHE}^{-} 45: \mathrm{CA}$ \\
\hline 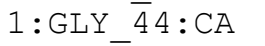 & $1: \mathrm{PHE}^{-} 46: \mathrm{CA}$ \\
\hline $1: \mathrm{PHE}_{4} 45: \mathrm{CA}$ & $1:$ TYR_47:CA \\
\hline 1: $\mathrm{PHE}^{-} 46: \mathrm{CA}$ & $1: \mathrm{THR}^{-} 48: \mathrm{CA}$ \\
\hline 1:TYR_47:CA & $1:$ LYST_ $49: \mathrm{CA}$ \\
\hline 1:THR_48:CA & $1: \mathrm{PRO}=\overline{5} 0: \mathrm{CA}$ \\
\hline
\end{tabular}

$\begin{array}{lllll}4.700 & 7.200 & 1.00 & 1.00 & 1000.000 \\ 4.700 & 7.200 & 1.00 & 1.00 & 1000.000 \\ 4.700 & 7.200 & 1.00 & 1.00 & 1000.000 \\ 4.700 & 7.200 & 1.00 & 1.00 & 1000.000 \\ 4.700 & 7.200 & 1.00 & 1.00 & 1000.000 \\ 4.700 & 7.200 & 1.00 & 1.00 & 1000.000 \\ 4.700 & 7.200 & 1.00 & 1.00 & 1000.000 \\ 4.700 & 7.200 & 1.00 & 1.00 & 1000.000 \\ 4.700 & 7.200 & 1.00 & 1.00 & 1000.000 \\ 4.700 & 7.200 & 1.00 & 1.00 & 1000.000 \\ 4.700 & 7.200 & 1.00 & 1.00 & 1000.000 \\ 4.700 & 7.200 & 1.00 & 1.00 & 1000.000 \\ 4.700 & 7.200 & 1.00 & 1.00 & 1000.000 \\ 4.700 & 7.200 & 1.00 & 1.00 & 1000.000 \\ 4.700 & 7.200 & 1.00 & 1.00 & 1000.000 \\ 4.700 & 7.200 & 1.00 & 1.00 & 1000.000 \\ 4.700 & 7.200 & 1.00 & 1.00 & 1000.000 \\ 4.700 & 7.200 & 1.00 & 1.00 & 1000.000 \\ 4.700 & 7.200 & 1.00 & 1.00 & 1000.000 \\ 4.700 & 7.200 & 1.00 & 1.00 & 1000.000 \\ 4.700 & 7.200 & 1.00 & 1.00 & 1000.000 \\ 4.700 & 7.200 & 1.00 & 1.00 & 1000.000 \\ 4.700 & 7.200 & 1.00 & 1.00 & 1000.000 \\ 4.700 & 7.200 & 1.00 & 1.00 & 1000.000 \\ 4.700 & 7.200 & 1.00 & 1.00 & 1000.000 \\ 4.700 & 7.200 & 1.00 & 1.00 & 1000.000 \\ 4.700 & 7.200 & 1.00 & 1.00 & 1000.000 \\ 4.700 & 7.200 & 1.00 & 1.00 & 1000.000 \\ 4.700 & 7.200 & 1.00 & 1.00 & 1000.000 \\ 4.700 & 7.200 & 1.00 & 1.00 & 1000.000 \\ 4.700 & 7.200 & 1.00 & 1.00 & 1000.000 \\ 4.700 & 7.200 & 1.00 & 1.00 & 1000.000 \\ 4.700 & 7.200 & 1.00 & 1.00 & 1000.000 \\ 4.700 & 7.200 & 1.00 & 1.00 & 1000.000 \\ 4.700 & 7.200 & 1.00 & 1.00 & 1000.000 \\ 4.700 & 7.200 & 1.00 & 1.00 & 1000.000 \\ 4.700 & 7.200 & 1.00 & 1.00 & 1000.000 \\ 4.700 & 7.200 & 1.00 & 1.00 & 1000.000 \\ 4.700 & 7.200 & 1.00 & 1.00 & 1000.000 \\ 4.700 & 7.200 & 1.00 & 1.00 & 1000.000 \\ 4.700 & 7.200 & 1.00 & 1.00 & 1000.000 \\ 4.700 & 7.200 & 1.00 & 1.00 & 1000.000 \\ 4.700 & 7.200 & 1.00 & 1.00 & 1000.000 \\ 4.700 & 7.200 & 1.00 & 1.00 & 1000.000 \\ 4.700 & 7.200 & 1.00 & 1.00 & 1000.000 \\ 4.700 & 7.200 & 1.00 & 1.00 & 1000.000\end{array}$




\begin{tabular}{|c|c|c|c|c|c|c|}
\hline $1:$ SER_12:0 & $1:$ GLN_15:HN & 2.300 & 2.600 & 10.00 & 10.00 & 1000.000 \\
\hline 1:SER_12:0 & $1:$ GLN_$^{-} 15: \mathrm{N}$ & 2.700 & 3.300 & 10.00 & 10.00 & 1000.000 \\
\hline $1:$ SER_12:HN & 1:GLN_15:OE1 & 2.300 & 2.600 & 10.00 & 10.00 & 1000.000 \\
\hline $1: \mathrm{SER}^{-12: N}$ & $1:$ GLN $^{-1} 15:$ OE1 & 2.700 & 3.300 & 10.00 & 10.00 & 1000.000 \\
\hline 1:SER_12:0 & $1: \mathrm{LEU}^{-} 16: \mathrm{HN}$ & 2.300 & 2.600 & 10.00 & 10.00 & 1000.000 \\
\hline $1: \operatorname{SER}^{-1} 12: 0$ & $1: \operatorname{LEU}^{-1} 16: \mathrm{N}$ & 2.700 & 3.300 & 10.00 & 10.00 & 1000.000 \\
\hline $1: \mathrm{LEU}^{-1} 13: 0$ & $1: \mathrm{GLU}^{-} 17: \mathrm{HN}$ & 2.300 & 2.600 & 10.00 & 10.00 & 1000.000 \\
\hline $1:$ LEU_13:13:0 & $1: \mathrm{GLU}^{-}{ }_{-} 17: \mathrm{N}$ & 2.700 & 3.300 & 10.00 & 10.00 & 1000.000 \\
\hline 1:TYR_14:0 & $1: \mathrm{GLU}-17: \mathrm{HN}$ & 2.300 & 2.600 & 10.00 & 10.00 & 1000.000 \\
\hline 1:TYR_14:0 & $1: \mathrm{GLU}^{-} 17: \mathrm{N}$ & 2.700 & 3.300 & 10.00 & 10.00 & 1000.000 \\
\hline $1:$ GLN_15:0 & $1: A S N \_18: H N$ & 2.300 & 2.600 & 10.00 & 10.00 & 1000.000 \\
\hline $1:$ GLN $^{-1} 15: 0$ & $1: \mathrm{ASN}^{-1} 18: \mathrm{N}$ & 2.700 & 3.300 & 10.00 & 10.00 & 1000.000 \\
\hline 1:LEU_16:0 & 1:TYR_19:HN & 2.300 & 2.600 & 10.00 & 10.00 & 1000.000 \\
\hline $1:$ LEU_16 $16: 0$ & $1: \mathrm{TYR}_{-}^{-19: \mathrm{N}}$ & 2.700 & 3.300 & 10.00 & 10.00 & 1000.000 \\
\hline $1:$ GLU-_17:0 & $1:$ CYS_20:HN & 2.300 & 2.600 & 10.00 & 10.00 & 1000.000 \\
\hline $1:$ GLU- $17: 0$ & $1:$ CYS_20:N & 2.700 & 3.300 & 10.00 & 10.00 & 1000.000 \\
\hline $1:$ GLY_Z 4 4:O & $1:$ ASNC $21:$ HN & 2.300 & 2.600 & 10.00 & 10.00 & 1000.000 \\
\hline $1: \mathrm{GLY}^{-} 44: 0$ & $1:$ ASNC $^{-} 21: N$ & 2.700 & 3.300 & 10.00 & 10.00 & 1000.000 \\
\hline 1:GLN_25:0 & $1:$ CYS_I $1:$ HN & 2.300 & 2.600 & 10.00 & 10.00 & 1000.000 \\
\hline $1:$ GLN_25:0 & $1:$ CYS_11:N & 2.700 & 3.300 & 10.00 & 10.00 & 1000.000 \\
\hline 1:CYS_28:0 & $1: \mathrm{ASP}-\quad 31: \mathrm{HN}$ & 2.300 & 2.600 & 10.00 & 10.00 & 1000.000 \\
\hline $1:$ CYS_28:0 & $1: \mathrm{ASP}-31: \mathrm{N}$ & 2.700 & 3.300 & 10.00 & 10.00 & 1000.000 \\
\hline 1:ALA_29:0 & $1:$ LEU_ $32: H N$ & 2.300 & 2.600 & 10.00 & 10.00 & 1000.000 \\
\hline 1:ALA 29:0 & $1:$ LEU $32: N$ & 2.700 & 3.300 & 10.00 & 10.00 & 1000.000 \\
\hline 1:ALA_29:0 & $1: \mathrm{VAL}^{-} 33: \mathrm{HN}$ & 2.300 & 2.600 & 10.00 & 10.00 & 1000.000 \\
\hline 1:ALA_29:0 & $1: \mathrm{VAL}^{-} 33: \mathrm{N}$ & 2.700 & 3.300 & 10.00 & 10.00 & 1000.000 \\
\hline $1:$ SER_30:0 & $1: \mathrm{GLU}-\quad 34: \mathrm{HN}$ & 2.300 & 2.600 & 10.00 & 10.00 & 1000.000 \\
\hline 1:SER_30:0 & $1: G L U-34: \mathrm{N}$ & 2.700 & 3.300 & 10.00 & 10.00 & 1000.000 \\
\hline $1:$ ASP-_31:0 & 1:ALA_35:HN & 2.300 & 2.600 & 10.00 & 10.00 & 1000.000 \\
\hline $1: \mathrm{ASP}-31: 0$ & $1:$ ALA $35: \mathrm{N}$ & 2.700 & 3.300 & 10.00 & 10.00 & 1000.000 \\
\hline $1:$ LEU_Z $32: 0$ & $1: \mathrm{LEU} 36: \mathrm{HN}$ & 2.300 & 2.600 & 10.00 & 10.00 & 1000.000 \\
\hline $1:$ LEU_32:0 & $1:$ LEU_36 $3: \mathrm{N}$ & 2.700 & 3.300 & 10.00 & 10.00 & 1000.000 \\
\hline $1: V A L \_33: 0$ & 1:TYR_37:HN & 2.300 & 2.600 & 10.00 & 10.00 & 1000.000 \\
\hline $1:$ VAL_33:0 & $1:$ TYR_37:N & 2.700 & 3.300 & 10.00 & 10.00 & 1000.000 \\
\hline $1:$ GLU-_34:0 & $1: \mathrm{LEU} \_38: \mathrm{HN}$ & 2.300 & 2.600 & 10.00 & 10.00 & 1000.000 \\
\hline $1:$ GLU- $34: 0$ & $1:$ LEU_38:N & 2.700 & 3.300 & 10.00 & 10.00 & 1000.000 \\
\hline 1:ALA_35:0 & $1: \mathrm{VAL} \_39: \mathrm{HN}$ & 2.300 & 2.600 & 10.00 & 10.00 & 1000.000 \\
\hline 1:ALA_35:0 & $1: \mathrm{VAL} \_39: \mathrm{N}$ & 2.700 & 3.300 & 10.00 & 10.00 & 1000.000 \\
\hline $1:$ LEU_36:0 & $1:$ CYS_4 $40: H N$ & 2.300 & 2.600 & 10.00 & 10.00 & 1000.000 \\
\hline $1:$ LEU_36:0 & $1:$ CYS_4 $40: N$ & 2.700 & 3.300 & 10.00 & 10.00 & 1000.000 \\
\hline 1:TYR_37:0 & $1:$ GLY_41:HN & 2.300 & 2.600 & 10.00 & 10.00 & 1000.000 \\
\hline 1:TYR_37:0 & $1: G L Y \_41: N$ & 2.700 & 3.300 & 10.00 & 10.00 & 1000.000 \\
\hline $1:$ CYS_6:0 & $1:$ LEU_27:HN & 2.300 & 2.600 & 10.00 & 10.00 & 1000.000 \\
\hline $\begin{array}{l}1: \text { CYS_6 } 6: 0 \\
!\end{array}$ & $1:$ LEU_27:N & 2.700 & 3.300 & 10.00 & 10.00 & 1000.000 \\
\hline \#NOE_distance & & & & & & \\
\hline $\begin{array}{l}1: \text { ILE_ } 2: H A \\
1000.000 \quad 0.00\end{array}$ & $1:$ GLN_5:HB* & -1.000 & 6.000 & 5.000 & 10.00 & 10.00 \\
\hline $\begin{array}{l}1: \mathrm{VAL} 3: \mathrm{HA} \\
1000.000 \quad 0.00\end{array}$ & $1: \mathrm{CYS}_{-} 7: \mathrm{HA}$ & -1.000 & 4.300 & 3.000 & 10.00 & 10.00 \\
\hline $\begin{array}{l}1: \text { VAL } 3: \text { HA } \\
1000.000 \quad 0.00\end{array}$ & $1:$ CYS_ $7: \mathrm{HB}^{*}$ & $-1 \cdot 0$ & 6.000 & 5.000 & 10.00 & 10.00 \\
\hline $\begin{array}{l}1: \mathrm{VAL} 3: \mathrm{HA} \\
1000.000 \quad 0.00\end{array}$ & $2: H D 1 *$ & -1.000 & 4.400 & 3.000 & 10.00 & 10.00 \\
\hline $\begin{array}{l}1: \mathrm{VAL} 3: \mathrm{HA} \\
1000 . \overline{0} 00 \quad 0.00\end{array}$ & $1:$ LEU_32:HD2* & -1.000 & 5.300 & 3.000 & 10.00 & 10.00 \\
\hline
\end{tabular}


$1:$ VAL $3: \mathrm{HN}$

$1000 . \overline{0} 00 \quad 0.00$

$1:$ VAL $3: \mathrm{HN}$

$1000.000 \quad 0.00$

$1: \mathrm{GLU}-4: \mathrm{HA}$

$1000.0 \overline{0} 0 \quad 0.00$

$1: \mathrm{GLU}-4: \mathrm{HN}$

$1000.0 \overline{0} 0 \quad 0.00$

$1: \mathrm{GLU}-4: \mathrm{HN}$

$1000.0 \overline{0} 0 \quad 0.00$

$1: \mathrm{GLU}-4: \mathrm{HN}$

$1000.0 \overline{0} 0 \quad 0.00$

$1: \mathrm{GLU}-4: \mathrm{HN}$

$1000.0 \overline{0} 0 \quad 0.00$

$1:$ GLN $5: \mathrm{HA}$

$1000 . \overline{0} 00 \quad 0.00$

1 : GLN $5: \mathrm{HN}$

$1000 . \overline{0} 00 \quad 0.00$

$1:$ GLN $5: \mathrm{HN}$

$1000 . \overline{0} 00 \quad 0.00$

1 :CYS 6:HB*

$1000 . \overline{0} 00 \quad 0.00$

$1: \mathrm{CYS} \quad 6: \mathrm{HB}^{*}$

$1000 . \overline{0} 00 \quad 0.00$

$1: \mathrm{CYS} \quad 6: \mathrm{HN}$

$1000 . \overline{0} 00 \quad 0.00$

1 :CYS $6: \mathrm{HN}$

$1000 . \overline{0} 00 \quad 0.00$

$1:$ CYS $6: \mathrm{HN}$

$1000 . \overline{0} 00 \quad 0.00$

$1:$ CYS $6: \mathrm{HN}$

$1000 . \overline{0} 00 \quad 0.00$

$1:$ CYS $6: \mathrm{HN}$

$1000 . \overline{0} 00 \quad 0.00$

$1:$ CYS $6: \mathrm{HN}$

$1000 . \overline{0} 00 \quad 0.00$

$1:$ CYS $6: \mathrm{HN}$

$1000 . \overline{0} 00 \quad 0.00$

$1:$ CYS $6: \mathrm{HN}$

$1000 . \overline{0} 00 \quad 0.00$

$1: \mathrm{CYS} \quad 7: \mathrm{HA}$

$1000 . \overline{0} 00 \quad 0.00$

$1:$ CYS $7: \mathrm{HA}$

$1000 . \overline{0} 00 \quad 0.00$

1 : CYS $7: \mathrm{HA}$

$1000 . \overline{0} 00 \quad 0.00$

1 :CYS $7: \mathrm{HB}^{*}$

$1000 . \overline{0} 00 \quad 0.00$

$1:$ CYS $7: \mathrm{HB}$ *

$1000 . \overline{0} 00 \quad 0.00$

$1:$ CYS $7: \mathrm{HN}$

$1000 . \overline{0} 00 \quad 0.00$

$1:$ CYS $7: \mathrm{HN}$

$1000 . \overline{0} 00 \quad 0.00$

1 : CYS $7: \mathrm{HN}$

$1000 . \overline{0} 00 \quad 0.00$

\begin{tabular}{|c|c|c|c|c|c|}
\hline $1:$ ILE_2:HG2* & -1.000 & 3.700 & 3.000 & 10.00 & 10.00 \\
\hline $1:$ GLU-_4 $4: \mathrm{HB}^{*}$ & -1.000 & 6.000 & 5.000 & 10.00 & 10.00 \\
\hline $1:$ THR_8:HB & -1.000 & 3.400 & 3.000 & 10.00 & 10.00 \\
\hline $1:$ VAL_3:HA & -1.000 & 5.000 & 3.000 & 10.00 & 10.00 \\
\hline $1:$ VAL_3:HB & -1.000 & 2.700 & 2.700 & 10.00 & 10.00 \\
\hline $1:$ VAL_3 $:$ HGI* & -1.000 & 4.400 & 3.000 & 10.00 & 10.00 \\
\hline $1:$ VAL_3 $:$ HG2* & -1.000 & 4.400 & 3.000 & 10.00 & 10.00 \\
\hline $1: \mathrm{GLU}-\_4: \mathrm{HA}$ & -1.000 & 5.000 & 3.000 & 10.00 & 10.00 \\
\hline $1: \mathrm{GLU}-\_4: \mathrm{HA}$ & -1.000 & 4.300 & 3.000 & 10.00 & 10.00 \\
\hline $1: \mathrm{GLU}-{ }^{\prime} 4: \mathrm{HB}^{*}$ & -1.000 & 6.000 & 5.000 & 10.00 & 10.00 \\
\hline $1:$ VAL_3:HG1* & -1.000 & 6.000 & 3.000 & 10.00 & 10.00 \\
\hline $1:$ VAL_3 $:$ HG $2 *$ & -1.000 & 6.500 & 3.000 & 10.00 & 10.00 \\
\hline $1:$ VAL_3:HA & -1.000 & 3.400 & 3.000 & 10.00 & 10.00 \\
\hline $1:$ GLU-_ $4: \mathrm{HG}^{*}$ & -1.000 & 6.000 & 5.000 & 10.00 & 10.00 \\
\hline $1:$ GLN_5:HB* & -1.000 & 4.400 & 3.000 & 10.00 & 10.00 \\
\hline $1:$ GLN_5:HG* & -1.000 & 6.000 & 5.000 & 10.00 & 10.00 \\
\hline $1:$ CYS_ $7: \mathrm{HA}$ & -1.000 & 5.000 & 3.000 & 10.00 & 10.00 \\
\hline $1: \mathrm{CYS} 7: \mathrm{HB}$ * & -1.000 & 6.000 & 5.000 & 10.00 & 10.00 \\
\hline $1:$ CYS_7 $: \mathrm{HN}$ & -1.000 & 3.400 & 3.000 & 10.00 & 10.00 \\
\hline $1:$ SER_9:HB* & -1.000 & 6.000 & 5.000 & 10.00 & 10.00 \\
\hline $1:$ HIS_26:HB* & -1.000 & 6.000 & 5.000 & 10.00 & 10.00 \\
\hline $1:$ LEU_32:HD1 * & -1.000 & 6.000 & 5.000 & 10.00 & 10.00 \\
\hline $1:$ LEU_32:HD2 * & -1.000 & 6.500 & 5.500 & 10.00 & 10.00 \\
\hline $1:$ LEU_32:HD1* & -1.000 & 7.000 & 5.000 & 10.00 & 10.00 \\
\hline $1:$ LEU_32:HD2* & -1.000 & 7.000 & 5.000 & 10.00 & 10.00 \\
\hline $1: \mathrm{GLU}-\_4: \mathrm{HA}$ & -1.000 & 3.700 & 3.000 & 10.00 & 10.00 \\
\hline $1: \mathrm{GLU}-\_4: \mathrm{HG}^{*}$ & -1.000 & 6.000 & 5.000 & 10.00 & 10.00 \\
\hline $1:$ CYS_6:HA & -1.000 & 5.000 & 3.000 & 10.00 & 10.00 \\
\hline
\end{tabular}




\begin{tabular}{|c|c|c|c|c|c|c|}
\hline $\begin{array}{l}1: \text { CYS } 7: \mathrm{HN} \\
1000.000 \quad 0.00\end{array}$ & $1:$ CYS_ $6: \mathrm{HB}^{*}$ & -1.000 & 6.000 & 5.000 & 10.00 & 10.00 \\
\hline $\begin{array}{l}1: \text { THR_8:HN } \\
1000 . \overline{0} 00 \quad 0.00\end{array}$ & $1:$ GLN_5:HA & -1.000 & 5.000 & 3.000 & 10.00 & 10.00 \\
\hline $\begin{array}{l}: \text { THR_8:HN } \\
000 . \overline{0} 00 \quad 0.00\end{array}$ & $1:$ CYS_7 : HA & -1.000 & 5.000 & 3.000 & 10.00 & 10.00 \\
\hline $\begin{array}{l}: \mathrm{SER} 9: \mathrm{HN} \\
000 . \overline{0} 00 \quad 0.00\end{array}$ & 1:ILE_10:HGI* & -1.000 & 4.400 & 3.000 & 10.00 & 10.00 \\
\hline $\begin{array}{l}: \text { CYS } 11: \mathrm{HA} \\
000.000 \quad 0.00\end{array}$ & 1:ILE_10:HG2* & -1.000 & 4.400 & 3.000 & 10.00 & 10.00 \\
\hline $\begin{array}{l}: \text { CYS } 11: \mathrm{HB}^{*} \\
000 . \overline{0} 00 \quad 0.00\end{array}$ & $1:$ LEU_27:HB* & -1.000 & 7.000 & 5.000 & 10.00 & 10.00 \\
\hline $\begin{array}{l}: \text { CYS } 11: \mathrm{HB}^{*} \\
000.000 \quad 0.00\end{array}$ & 1:LEU_27:HD1* & -1.000 & 7.000 & 5.000 & 10.00 & 10.00 \\
\hline $\begin{array}{l}: \text { CYS } 11: \mathrm{HB}^{*} \\
000.000 \quad 0.00\end{array}$ & 1:LEU_27:HD2* & -1.000 & 7.000 & 5.000 & 10.00 & 10.00 \\
\hline $\begin{array}{l}: \text { SER_12:HN } \\
000 . \overline{0} 00 \quad 0.00\end{array}$ & $1:$ CYS_11:HA & -1.000 & 5.000 & 3.000 & 10.00 & 10.00 \\
\hline $\begin{array}{l}: \text { LEU } 13: \text { HA } \\
000.000 \quad 0.00\end{array}$ & $1:$ LEU_16:HB* & -1.000 & 4.400 & 3.000 & 10.00 & 10.00 \\
\hline $\begin{array}{l}1: \mathrm{LEU} 13: \mathrm{HN} \\
1000.000 \quad 0.00\end{array}$ & $1: \mathrm{SER} \_12: \mathrm{HN}$ & -1.000 & 5.000 & 3.000 & 10.00 & 10.00 \\
\hline $\begin{array}{l}1: \mathrm{LEU} 13: \mathrm{HN} \\
1000 . \overline{0} 00 \quad 0.00\end{array}$ & $1:$ TYR_14:HB* & -1.000 & 6.000 & 5.000 & 10.00 & 10.00 \\
\hline $\begin{array}{l}1: \mathrm{LEU} 13: \mathrm{HN} \\
1000.000 \quad 0.00\end{array}$ & $1:$ TYR_14:HN & -1.000 & 3.400 & 3.000 & 10.00 & 10.00 \\
\hline $\begin{array}{l}1: \text { TYR_14:HA } \\
1000.000 \quad 0.00\end{array}$ & $1: \mathrm{GLU}-\_17: \mathrm{HB} *$ & -1.000 & 6.000 & 5.000 & 10.00 & 10.00 \\
\hline $\begin{array}{l}1: \text { TYR_1 } 14: \mathrm{HB}^{*} \\
1000 . \overline{0} 00 \quad 0.00\end{array}$ & $1:$ GLN_15:HG* & -1.000 & 7.000 & 5.000 & 10.00 & 10.00 \\
\hline $\begin{array}{l}1: \text { TYR_14:HD* } \\
1000.000 \quad 0.00\end{array}$ & $1:$ GLN_15:HA & -1.000 & 7.000 & 5.000 & 10.00 & 10.00 \\
\hline $\begin{array}{l}1: \text { TYR_14:HD* } \\
1000.000 \quad 0.00\end{array}$ & $1:$ GLN_15:HG* & -1.000 & 8.000 & 5.000 & 10.00 & 10.00 \\
\hline $\begin{array}{l}1: \text { TYR_14:HD* } \\
1000.000 \quad 0.00\end{array}$ & $1: \mathrm{GLU}-\_17: \mathrm{HG}^{*}$ & -1.000 & 8.000 & 5.000 & 10.00 & 10.00 \\
\hline $\begin{array}{l}1: \text { TYR_14:HN } \\
1000.000 \quad 0.00\end{array}$ & $1: \mathrm{SER} \_12: \mathrm{HA}$ & -1.000 & 5.000 & 3.000 & 10.00 & 10.00 \\
\hline $\begin{array}{l}1: \text { TYR_14:HN } \\
1000.000 \quad 0.00\end{array}$ & $1:$ SER_12:HB* & -1.000 & 6.000 & 5.000 & 10.00 & 10.00 \\
\hline $\begin{array}{l}1: \text { TYR_14:HN } \\
1000.000 \quad 0.00\end{array}$ & $1:$ LEU_13:HA & -1.000 & 5.000 & 3.000 & 10.00 & 10.00 \\
\hline $\begin{array}{l}1: \text { TYR } 14: H N \\
1000.000 \quad 0.00\end{array}$ & $1:$ LEU_13:HB* & -1.000 & 6.000 & 5.000 & 10.00 & 10.00 \\
\hline $\begin{array}{l}1: \text { TYR_14:HN } \\
1000.000 \quad 0.00\end{array}$ & $1:$ LEU_13 $:$ HG & -1.000 & 5.000 & 3.000 & 10.00 & 10.00 \\
\hline $\begin{array}{l}1: \text { TYR } 14: \text { HN } \\
1000.000 \quad 0.00\end{array}$ & $1:$ GLN_15:HN & -1.000 & 3.400 & 3.000 & 10.00 & 10.00 \\
\hline $\begin{array}{l}1: \text { GLN } 15: \mathrm{HB}^{*} \\
1000.000 \quad 0.00\end{array}$ & $1:$ CYS_11:HB* & -1.000 & 6.300 & 4.300 & 10.00 & 10.00 \\
\hline $\begin{array}{l}1: \text { GLN } 15: \text { HN } \\
1000.000 \quad 0.00\end{array}$ & $1:$ LEU_13:HA & -1.000 & 5.000 & 3.000 & 10.00 & 10.00 \\
\hline $\begin{array}{l}1: \text { GLN } 15: \text { HN } \\
1000.000 \quad 0.00\end{array}$ & 1:TYR_14:HA & -1.000 & 3.400 & 3.000 & 10.00 & 10.00 \\
\hline $\begin{array}{l}1: \text { GLN } 15: \text { HN } \\
1000.000 \quad 0.00\end{array}$ & $1:$ TYR_14:HB* & -1.000 & 3.700 & 3.000 & 10.00 & 10.00 \\
\hline
\end{tabular}


$1:$ GLN $15: \mathrm{HN}$

$1000 . \overline{0} 00 \quad 0.00$

$1:$ GLN $15: \mathrm{HN}$

$1000 . \overline{0} 00 \quad 0.00$

$1: \mathrm{LEU} 16: \mathrm{HN}$

$1000 . \overline{0} 00 \quad 0.00$

1 : LEU $16: \mathrm{HN}$

$1000 . \overline{0} 00 \quad 0.00$

$1:$ LEU_16:HN

$1000 . \overline{0} 00 \quad 0.00$

$1: \mathrm{LEU} 16: \mathrm{HN}$

$1000 . \overline{0} 00 \quad 0.00$

$1: \mathrm{LEU} 16: \mathrm{HN}$

$1000 . \overline{0} 00 \quad 0.00$

$1: \mathrm{LEU} 16: \mathrm{HN}$

$1000 . \overline{0} 00 \quad 0.00$

$1: \mathrm{LEU} 16: \mathrm{HN}$

$1000 . \overline{0} 00 \quad 0.00$

$1:$ LEU_16:HN

$1000 . \overline{0} 00 \quad 0.00$

$1: \mathrm{GLU}-17: \mathrm{HA}$

$1000.0 \overline{0} 0.00$

$1: \mathrm{GLU}-17$ :HA

$1000.0 \overline{0} 0 \quad 0.00$

$1: \mathrm{GLU}-17: \mathrm{HB}$ *

$1000.0 \overline{0} 0 \quad 0.00$

$1:$ GLU $-17: \mathrm{HB}^{*}$

$1000.0 \overline{0} 0 \quad 0.00$

$1: \mathrm{GLU}-17: \mathrm{HG}^{*}$

$1000.0 \overline{0} 0 \quad 0.00$

$1:$ GLU $-17: \mathrm{HG}^{*}$

$1000.0 \overline{0} 0 \quad 0.00$

$1: \mathrm{GLU}-17$ :HN

$1000.0 \overline{0} 0 \quad 0.00$

1 : GLU- 17 : HN

$1000.0 \overline{0} 0 \quad 0.00$

$1: \mathrm{GLU}-17: \mathrm{HN}$

$1000.0 \overline{0} 0 \quad 0.00$

$1: \mathrm{GLU}-17$ : HN

$1000.0 \overline{0} 0 \quad 0.00$

1 : GLU- 17 : HN

$1000.0 \overline{0} 0 \quad 0.00$

$1: \mathrm{GLU}-17: \mathrm{HN}$

$1000.0 \overline{0} 0 \quad 0.00$

$1: \mathrm{ASN} 18: \mathrm{HN}$

$1000 . \overline{0} 00 \quad 0.00$

$1: \mathrm{ASN} 18: \mathrm{HN}$

$1000 . \overline{0} 00 \quad 0.00$

$1: \mathrm{ASN} 18: \mathrm{HN}$

$1000 . \overline{0} 00 \quad 0.00$

1:ASN $18: \mathrm{HN}$

$1000 . \overline{0} 00 \quad 0.00$

$1:$ ASN_18:HN

$1000 . \overline{0} 00 \quad 0.00$

1:TYR 19: HA

$1000 . \overline{0} 00 \quad 0.00$

\begin{tabular}{|c|c|c|c|c|c|}
\hline $1: L E U \_16: H N$ & -1.000 & 2.700 & 2.700 & 10.00 & 10.00 \\
\hline $1: \mathrm{GLU}-\_17: \mathrm{HN}$ & -1.000 & 5.000 & 3.000 & 10.00 & 10.00 \\
\hline $1:$ LEU_13:HA & -1.000 & 3.400 & 3.000 & 10.00 & 10.00 \\
\hline $1: \mathrm{GLN}_{-} 15: \mathrm{HA}$ & -1.000 & 4.300 & 3.000 & 10.00 & 10.00 \\
\hline $1:$ GLN_15:HB* & -1.000 & 6.000 & 5.000 & 10.00 & 10.00 \\
\hline $1: \mathrm{GLN}_{1} 15: \mathrm{HG}^{\star}$ & -1.000 & 4.400 & 3.000 & 10.00 & 10.00 \\
\hline $1: \mathrm{GLU}-\_17: \mathrm{HA}$ & -1.000 & 5.000 & 3.000 & 10.00 & 10.00 \\
\hline $1: \mathrm{GLU}-\_17: \mathrm{HN}$ & -1.000 & 3.400 & 3.000 & 10.00 & 10.00 \\
\hline $1: \mathrm{VAL}$ 39:HGI* & -1.000 & 6.000 & 5.000 & 10.00 & 10.00 \\
\hline $1:$ VAL_39:HG2* & -1.000 & 6.000 & 5.000 & 10.00 & 10.00 \\
\hline $1: \mathrm{VAL} \_39: \mathrm{HGI}$ * & -1.000 & 6.000 & 5.000 & 10.00 & 10.00 \\
\hline $1:$ VAL_39:HG2* & -1.000 & 6.000 & 5.000 & 10.00 & 10.00 \\
\hline $1: \mathrm{VAL} \_39: \mathrm{HGI}$ * & -1.000 & 5.400 & 3.000 & 10.00 & 10.00 \\
\hline $1: \mathrm{VAL} \_39: \mathrm{HG} 2$ * & -1.000 & 5.400 & 3.000 & 10.00 & 10.00 \\
\hline $1: \mathrm{VAL} \_39: \mathrm{HGI}$ * & -1.000 & 5.400 & 3.000 & 10.00 & 10.00 \\
\hline $1: V A L \_39: H G 2$ * & -1.000 & 5.400 & 3.000 & 10.00 & 10.00 \\
\hline $1: T Y R \_14: H A$ & -1.000 & 3.400 & 3.000 & 10.00 & 10.00 \\
\hline $1:$ LEU_16:HB* & -1.000 & 4.400 & 3.000 & 10.00 & 10.00 \\
\hline $1: \mathrm{LEU}_{-} 16: \mathrm{HD} 1$ * & -1.000 & 4.400 & 3.000 & 10.00 & 10.00 \\
\hline $1: \mathrm{LEU}_{-} 16: \mathrm{HD} 2$ * & -1.000 & 5.300 & 3.000 & 10.00 & 10.00 \\
\hline $1: L E L_{-} 16: \mathrm{HG}$ & -1.000 & 5.000 & 3.000 & 10.00 & 10.00 \\
\hline $1: \mathrm{CYS} \_20: \mathrm{HN}$ & -1.000 & 5.500 & 3.000 & 10.00 & 10.00 \\
\hline $1: \mathrm{GLN}_{2} 15: \mathrm{HA}$ & -1.000 & 4.300 & 3.000 & 10.00 & 10.00 \\
\hline $1:$ LEU_1 $6: \mathrm{HB} *$ & -1.000 & 6.000 & 5.000 & 10.00 & 10.00 \\
\hline $1: \mathrm{GLU}-\_17: \mathrm{HA}$ & -1.000 & 3.400 & 3.000 & 10.00 & 10.00 \\
\hline $1: \mathrm{GLU}-\_17: \mathrm{HG}^{*}$ & -1.000 & 6.000 & 5.000 & 10.00 & 10.00 \\
\hline $1: T Y R \_19: H N$ & -1.000 & 2.700 & 2.700 & 10.00 & 10.00 \\
\hline 1:ILE 2:HG1* & -1.000 & 6.000 & 5.000 & 10.00 & 10.00 \\
\hline
\end{tabular}


1:TYR 19:HB*

$1000 . \overline{0} 00 \quad 0.00$

1: TYR 19: HB *

$1000 . \overline{0} 00 \quad 0.00$

1:TYR_19: HB*

$1000 . \overline{0} 00 \quad 0.00$

1:TYR 19:HD*

$1000 . \overline{0} 00 \quad 0.00$

1:TYR 19:HD*

$1000 . \overline{0} 00 \quad 0.00$

1 :TYR $19: \mathrm{HD}^{*}$

$1000 . \overline{0} 00 \quad 0.00$

1:TYR 19:HD*

$1000 . \overline{0} 00 \quad 0.00$

1:TYR_19:HD*

$1000 . \overline{0} 00 \quad 0.00$

1:TYR 19: HE*

$1000 . \overline{0} 00 \quad 0.00$

1:TYR_19: HE*

$1000 . \overline{0} 00 \quad 0.00$

1:TYR_19: HE*

$1000 . \overline{0} 00 \quad 0.00$

1:TYR 19: HE*

$1000 . \overline{0000} 0.00$

1:TYR 19: HE*

$1000 . \overline{0} 00 \quad 0.00$

1:TYR $19: \mathrm{HE}^{*}$

$1000 . \overline{0} 00 \quad 0.00$

1:TYR 19: HE*

$1000 . \overline{0} 00 \quad 0.00$

1:TYR_19:HN

$1000 . \overline{0} 00 \quad 0.00$

1:TYR 19:HN

$1000 . \overline{0} 00 \quad 0.00$

$1:$ TYR_19:HN

$1000 . \overline{0} 00 \quad 0.00$

1:TYR_19:HN

$1000 . \overline{0} 00 \quad 0.00$

1:TYR 19: HN

$1000 . \overline{0} 00 \quad 0.00$

$1:$ TYR_19:HN

$1000 . \overline{0} 00 \quad 0.00$

$1:$ TYR 19:HN

$1000 . \overline{0} 00 \quad 0.00$

1 : CYS 20 : HA

$1000 . \overline{0} 00 \quad 0.00$

$1:$ CYS_20:HN

$1000 . \overline{0} 00 \quad 0.00$

1:ASNC 21 :HD2 *

$1000.0 \overline{0} 00.00$

1:ASNC 21:HD2*

$1000.0 \overline{0} 0 \quad 0.00$

1:ASNC_21:HN

$1000.0 \overline{0} 0 \quad 0.00$

1:ASNC 21:HN

$1000.0 \overline{0} 0 \quad 0.00$

\begin{tabular}{|c|c|c|c|c|c|}
\hline $1:$ ILE_2:HG1* & -1.000 & 7.000 & 5.000 & 10.00 & 10.00 \\
\hline $1:$ LEU_36:HD1* & -1.000 & 7.000 & 5.000 & 10.00 & 10.00 \\
\hline $1:$ LEU_36:HD2 * & -1.000 & 7.000 & 5.000 & 10.00 & 10.00 \\
\hline $1:$ ILE_2:HD1* & -1.000 & 5.700 & 3.000 & 10.00 & 10.00 \\
\hline 1:ILE_2:HG2* & -1.000 & 6.400 & 3.400 & 10.00 & 10.00 \\
\hline $1:$ LEU_16:HA & -1.000 & 4.700 & 3.000 & 10.00 & 10.00 \\
\hline $1:$ LEU_36:HD1* & -1.000 & 5.700 & 3.000 & 10.00 & 10.00 \\
\hline $1:$ LEU_36:HD2* & -1.000 & 5.700 & 3.000 & 10.00 & 10.00 \\
\hline $1:$ ILE_2:HB & -1.000 & 7.000 & 5.000 & 10.00 & 10.00 \\
\hline 1:ILE_2:HD1* & -1.000 & 5.700 & 3.000 & 10.00 & 10.00 \\
\hline $1:$ ILE_2:HG2* & -1.000 & 5.700 & 3.000 & 10.00 & 10.00 \\
\hline $1:$ LEU_16:HA & -1.000 & 4.700 & 3.000 & 10.00 & 10.00 \\
\hline $1:$ LEU_16:HB* & -1.000 & 8.000 & 5.000 & 10.00 & 10.00 \\
\hline $1:$ LEU_36:HDI * & -1.000 & 6.400 & 3.400 & 10.00 & 10.00 \\
\hline $1:$ LEU_36:HD2* & -1.000 & 6.400 & 3.400 & 10.00 & 10.00 \\
\hline $1:$ LEU_16:HA & -1.000 & 4.300 & 3.000 & 10.00 & 10.00 \\
\hline $1: \mathrm{LEU}_{-} 16: \mathrm{HD} 1$ * & -1.000 & 6.000 & 5.000 & 10.00 & 10.00 \\
\hline $1: \mathrm{GLU}-\_17: \mathrm{HA}$ & -1.000 & 3.400 & 3.000 & 10.00 & 10.00 \\
\hline $1: \mathrm{ASN}_{-} 18: \mathrm{HA}$ & -1.000 & 3.400 & 3.000 & 10.00 & 10.00 \\
\hline $1: \mathrm{ASN}_{1} 18: \mathrm{HB}^{\star}$ & -1.000 & 4.400 & 3.000 & 10.00 & 10.00 \\
\hline $1: \mathrm{LEU}_{-} 36: \mathrm{HD} 1$ * & -1.000 & 5.300 & 3.000 & 10.00 & 10.00 \\
\hline $1: \mathrm{LEU}_{-} 36: \mathrm{HD} 2$ * & -1.000 & 5.300 & 3.000 & 10.00 & 10.00 \\
\hline $1: \mathrm{PHE}_{4} 45: \mathrm{HB}^{*}$ & -1.000 & 6.000 & 5.000 & 10.00 & 10.00 \\
\hline $1: \mathrm{PHE}_{4} 45: \mathrm{HA}$ & -1.000 & 5.000 & 3.000 & 10.00 & 10.00 \\
\hline $1: \mathrm{CYS} \_20: \mathrm{HB} *$ & -1.000 & 7.000 & 5.000 & 10.00 & 10.00 \\
\hline $1:$ GLY_4 $44: \mathrm{HA}^{*}$ & -1.000 & 7.000 & 5.000 & 10.00 & 10.00 \\
\hline $1:$ CYS_20:HA & -1.000 & 2.700 & 2.700 & 10.00 & 10.00 \\
\hline $1:$ CYS 20 & -1.000 & 4.400 & 3.000 & 10.00 & 10.00 \\
\hline
\end{tabular}




\begin{tabular}{|c|c|c|c|c|c|c|}
\hline $\begin{array}{l}1: \operatorname{ASNC} 21: \mathrm{HN} \\
1000.000 \quad 0.00\end{array}$ & $1:$ CYS_4 $40: \mathrm{HB}^{*}$ & -1.000 & 5.500 & 5.000 & 10.00 & 10.00 \\
\hline $\begin{array}{l}: \text { ASNC } 21: \mathrm{HN} \\
000.0000 .00\end{array}$ & $1:$ PHE_ $45: \mathrm{HA}$ & -1.000 & 3.400 & 3.000 & 10.00 & 10.00 \\
\hline :PHEN_22:HB* & 1:LEU_13:HD1 * & -1.000 & 7.000 & 5.000 & 10.00 & 10.00 \\
\hline $000.0 \overline{0} 0 \quad 0.00$ & & & & & & \\
\hline $\begin{array}{l}: \text { PHEN_22:HB* } \\
000.000 \quad 0.00\end{array}$ & $1:$ LEU_13:HD2* & -1.000 & 7.000 & 5.000 & 10.00 & 10.00 \\
\hline $\begin{array}{l}: \text { PHEN_22:HD* } \\
000.0000 .00\end{array}$ & $1:$ LEU_13:HB* & -1.000 & 7.000 & 5.000 & 10.00 & 10.00 \\
\hline $\begin{array}{l}: \text { PHEN_22:HD* } \\
000.000 \quad 0.00\end{array}$ & $1:$ LEU_13:HD1* & -1.000 & 8.000 & 5.000 & 10.00 & 10.00 \\
\hline $\begin{array}{l}: \text { PHEN 22:HD* } \\
000.000 \quad 0.00\end{array}$ & $1:$ LEU_13:HD2 * & -1.000 & 8.000 & 5.000 & 10.00 & 10.00 \\
\hline $\begin{array}{l}: \text { PHEN_22:HD* } \\
000.0 \overline{0} 0.00\end{array}$ & $1: \mathrm{VAL} 223: \mathrm{HA}$ & -1.000 & 4.700 & 3.000 & 10.00 & 10.00 \\
\hline $\begin{array}{l}: \text { PHEN_22: } \mathrm{HE}^{\star} \\
000.0 \overline{0} 0.00\end{array}$ & $1:$ LEU_13:HB* & -1.000 & 8.000 & 5.000 & 10.00 & 10.00 \\
\hline $\begin{array}{l}: \text { PHEN_22: } \mathrm{HE}^{\star} \\
000.0 \overline{0} 0.00\end{array}$ & $1:$ LEU_13:HD1* & -1.000 & 8.000 & 5.000 & 10.00 & 10.00 \\
\hline $\begin{array}{l}: \text { PHEN_22: } \mathrm{HE}^{\star} \\
000.0 \overline{0} 0.00\end{array}$ & $1:$ LEU_13:HD2* & -1.000 & 8.000 & 5.000 & 10.00 & 10.00 \\
\hline $\begin{array}{l}1: \mathrm{VAL} 23: \mathrm{HN} \\
1000 . \overline{0} 00 \quad 0.00\end{array}$ & $1:$ PHEN_22:HA & -1.000 & 5.000 & 3.000 & 10.00 & 10.00 \\
\hline $\begin{array}{l}: \text { ASN_24:HA } \\
000.000 \quad 0.00\end{array}$ & $1:$ ILE_10:HG2* & -1.000 & 4.400 & 3.000 & 10.00 & 10.00 \\
\hline $\begin{array}{l}: \text { ASN_24:HA } \\
000.000 \quad 0.00\end{array}$ & $1:$ VAL_23:HGI * & -1.000 & 6.000 & 5.000 & 10.00 & 10.00 \\
\hline $\begin{array}{l}1: \mathrm{ASN}_{2} 24: \mathrm{HA} \\
1000 . \overline{0} 00 \quad 0.00\end{array}$ & 1:VAL_23:HG2* & -1.000 & 6.000 & 5.000 & 10.00 & 10.00 \\
\hline $\begin{array}{l}: \text { ASN_24:HB* } \\
000.000 \quad 0.00\end{array}$ & $1:$ ILE_10:HG2* & -1.000 & 5.400 & 3.000 & 10.00 & 10.00 \\
\hline $\begin{array}{l}1: \text { ASN_24:HD2* } \\
1000.000 \quad 0.00\end{array}$ & $1:$ VAL_23:HGI* & -1.000 & 7.000 & 5.000 & 10.00 & 10.00 \\
\hline $\begin{array}{l}1: \mathrm{ASN}_{2} 24: \mathrm{HD} 2 * \\
1000 . \overline{0} 00 \quad 0.00\end{array}$ & 1:VAL_23:HG2* & -1.000 & 7.000 & 5.000 & 10.00 & 10.00 \\
\hline $\begin{array}{l}1: \text { ASN } 24: H N \\
1000.000 \quad 0.00\end{array}$ & 1:ILE_10:HD1* & -1.000 & 6.000 & 5.000 & 10.00 & 10.00 \\
\hline $\begin{array}{l}1: \text { ASN_24:HN } \\
1000.000 \quad 0.00\end{array}$ & 1:ILE_10:HG2* & -1.000 & 6.000 & 5.000 & 10.00 & 10.00 \\
\hline $\begin{array}{l}1: \text { ASN_2 }_{2}: \mathrm{HN} \\
1000 . \overline{0} 00 \quad 0.00\end{array}$ & $1: \mathrm{VAL} 23: \mathrm{HA}$ & -1.000 & 2.700 & 2.700 & 10.00 & 10.00 \\
\hline $\begin{array}{l}1: \text { ASN_24:HN } \\
1000.000 \quad 0.00\end{array}$ & $1:$ VAL_23:HB & -1.000 & 4.300 & 3.000 & 10.00 & 10.00 \\
\hline $\begin{array}{l}1: \text { ASN_24:HN } \\
1000.000 \quad 0.00\end{array}$ & $1:$ VAL_23:HGI * & -1.000 & 4.400 & 3.000 & 10.00 & 10.00 \\
\hline $\begin{array}{l}1: \text { ASN_24:HN } \\
1000.000 \quad 0.00\end{array}$ & $1:$ VAL_23:HG2* & -1.000 & 4.400 & 3.000 & 10.00 & 10.00 \\
\hline $\begin{array}{l}1: \text { GLN_25:HN } \\
1000 . \overline{0} 00 \quad 0.00\end{array}$ & 1:ILE_10:HG2* & -1.000 & 4.400 & 3.000 & 10.00 & 10.00 \\
\hline $\begin{array}{l}1: \text { GLN_25:HN } \\
1000.000 \quad 0.00\end{array}$ & $1:$ VAL_23:HGI * & -1.000 & 6.000 & 5.000 & 10.00 & 10.00 \\
\hline $\begin{array}{l}1: \text { GLN_25:HN } \\
1000.000 \quad 0.00\end{array}$ & 1:VAL_23:HG2* & -1.000 & 6.000 & 5.000 & 10.00 & 10.00 \\
\hline $\begin{array}{l}1: \text { GLN } 25: \text { HN } \\
1000.000 \quad 0.00\end{array}$ & :ASN_24:HA & -1.000 & 2.700 & 2.700 & 10.00 & 10.00 \\
\hline
\end{tabular}


$1:$ GLN $25: \mathrm{HN}$

$1000 . \overline{0} 00 \quad 0.00$

1 :HIS 26:HA

$1000 . \overline{0} 00 \quad 0.00$

1 :HIS 26:HA

$1000 . \overline{0} 00 \quad 0.00$

1 :HIS 26: HA

$1000 . \overline{0} 00 \quad 0.00$

1:HIS 26:HE1

$1000 . \overline{0} 00 \quad 0.00$

1 :HIS 26:HE1

$1000 . \overline{0} 00 \quad 0.00$

1 :HIS $26: \mathrm{HE} 1$

$1000 . \overline{0} 00 \quad 0.00$

1:HIS 26:HE1

$1000 . \overline{0} 00 \quad 0.00$

$1: \mathrm{HIS} \quad 26: \mathrm{HN}$

$1000 . \overline{0} 00 \quad 0.00$

1 :HIS_26:HN

$1000 . \overline{0} 00 \quad 0.00$

$1:$ HIS 26:HN

$1000 . \overline{0} 00 \quad 0.00$

$1:$ LEU $27: \mathrm{HA}$

$1000 . \overline{0} 00 \quad 0.00$

$1:$ LEU $27:$ HA

$1000 . \overline{0} 00 \quad 0.00$

$1: \mathrm{LEU} 27$ : HA

$1000 . \overline{0} 00 \quad 0.00$

$1: \mathrm{LEU} 27: \mathrm{HN}$

$1000 . \overline{0} 00 \quad 0.00$

$1: \mathrm{LEU} 27: \mathrm{HN}$

$1000 . \overline{0} 00 \quad 0.00$

$1: \mathrm{LEU} \quad 27: \mathrm{HN}$

$1000 . \overline{0} 00 \quad 0.00$

1 :CYS_28:HA

$1000 . \overline{0} 00 \quad 0.00$

$1:$ CYS_28:HA

$1000 . \overline{0} 00 \quad 0.00$

1 :CYS $28: \mathrm{HA}$

$1000 . \overline{0} 00 \quad 0.00$

$1:$ CYS $28:$ HA

$1000 . \overline{0} 00 \quad 0.00$

1 :CYS 28:HA

$1000 . \overline{0} 00 \quad 0.00$

$1:$ CYS $28: \mathrm{HB}^{*}$

$1000 . \overline{0} 00 \quad 0.00$

1 : CYS $28: \mathrm{HN}$

$1000 . \overline{0} 00 \quad 0.00$

1 :CYS $28: \mathrm{HN}$

$1000 . \overline{0} 00 \quad 0.00$

$1:$ CYS $28: \mathrm{HN}$

$1000 . \overline{0} 00 \quad 0.00$

$1:$ CYS_28:HN

$1000 . \overline{0} 00 \quad 0.00$

1:ALA 29:HN

$1000 . \overline{0} 00 \quad 0.00$

\begin{tabular}{|c|c|c|c|c|c|}
\hline $1:$ ASN_24:HB* & -1.000 & 6.000 & 5.000 & 10.00 & 10.00 \\
\hline 1:ILE_10:HD1* & -1.000 & 4.400 & 3.000 & 10.00 & 10.00 \\
\hline $1:$ ILE_10:HG2* & -1.000 & 4.400 & 3.000 & 10.00 & 10.00 \\
\hline $1:$ LEU_27:HB* & -1.000 & 6.000 & 5.000 & 10.00 & 10.00 \\
\hline $1:$ SER_9:HA & -1.000 & 4.400 & 3.000 & 10.00 & 10.00 \\
\hline $1:$ ILE_10:HD1 * & -1.000 & 4.400 & 3.000 & 10.00 & 10.00 \\
\hline $1:$ ILE_10:HGI* & -1.000 & 6.000 & 5.000 & 10.00 & 10.00 \\
\hline 1:ILE_10:HG2* & -1.000 & 6.000 & 5.000 & 10.00 & 10.00 \\
\hline $1:$ GLN_25:HA & -1.000 & 2.700 & 2.700 & 10.00 & 10.00 \\
\hline $1:$ GLN_25:HG* & -1.000 & 6.000 & 5.000 & 10.00 & 10.00 \\
\hline $1:$ GLN_25:HN & -1.000 & 5.000 & 3.000 & 10.00 & 10.00 \\
\hline $1: \mathrm{ASP}-\_31: \mathrm{HB} *$ & -1.000 & 6.000 & 5.000 & 10.00 & 10.00 \\
\hline 1:LEU_32:HD1* & -1.000 & 4.400 & 3.000 & 10.00 & 10.00 \\
\hline $1:$ LEU_32:HD2* & -1.000 & 6.000 & 5.000 & 10.00 & 10.00 \\
\hline $1:$ CYS_7 $: \mathrm{HA}$ & -1.000 & 3.400 & 3.000 & 10.00 & 10.00 \\
\hline $1:$ HIS_26:HA & -1.000 & 2.700 & 2.700 & 10.00 & 10.00 \\
\hline $1:$ HIS_26:HB* & -1.000 & 6.000 & 5.000 & 10.00 & 10.00 \\
\hline $1: \mathrm{CYS}_{3} 7: \mathrm{HB}^{*}$ & -1.000 & 4.400 & 3.000 & 10.00 & 10.00 \\
\hline $1:$ ALA_29:HB* & -1.000 & 6.000 & 5.000 & 10.00 & 10.00 \\
\hline $1:$ LEU_32:HB* & -1.000 & 6.000 & 5.000 & 10.00 & 10.00 \\
\hline 1:LEU_32:HD1* & -1.000 & 4.400 & 3.000 & 10.00 & 10.00 \\
\hline $1:$ LEU_32:HD2* & -1.000 & 4.400 & 3.000 & 10.00 & 10.00 \\
\hline $1:$ ALA_29:HB* & -1.000 & 7.000 & 5.000 & 10.00 & 10.00 \\
\hline $1:$ LEU_27:HA & -1.000 & 2.700 & 2.700 & 10.00 & 10.00 \\
\hline $1:$ LEU_27:HDI* & -1.000 & 5.000 & 3.400 & 10.00 & 10.00 \\
\hline $1:$ LEU_27:HD2* & -1.000 & 5.000 & 3.400 & 10.00 & 10.00 \\
\hline $1: \mathrm{ASP}-\_31: \mathrm{HB} *$ & -1.000 & 4.400 & 3.000 & 10.00 & 10.00 \\
\hline $1:$ CYS_28:HA & -1.000 & 3.400 & 3.000 & 10.00 & 10.00 \\
\hline
\end{tabular}


1:ALA 29:HN

$1000 . \overline{0} 00 \quad 0.00$

1 :SER $30: \mathrm{HA}$

$1000 . \overline{0} 00 \quad 0.00$

1 :SER $30: \mathrm{HN}$

$1000 . \overline{0} 00 \quad 0.00$

$1: \mathrm{SER} 30: \mathrm{HN}$

$1000 . \overline{0} 00 \quad 0.00$

$1: \mathrm{ASP}-31: \mathrm{HA}$

$1000.0 \overline{0} 0 \quad 0.00$

$1: \mathrm{ASP}-31: \mathrm{HB}$ *

$1000.0 \overline{0} 00.00$

$1: \mathrm{ASP}-31: \mathrm{HB}$ *

$1000.0 \overline{0} 0 \quad 0.00$

$1: \mathrm{ASP}-31: \mathrm{HB}$ *

$1000.0 \overline{0} 0 \quad 0.00$

1:ASP- $31: \mathrm{HN}$

$1000.0 \overline{0} 0 \quad 0.00$

$1: \mathrm{ASP}-31: \mathrm{HN}$

$1000.0 \overline{0} 0 \quad 0.00$

$1: \mathrm{ASP}-31: \mathrm{HN}$

$1000.0 \overline{0} 00.00$

$1: \mathrm{ASP}-31: \mathrm{HN}$

$1000.0 \overline{0} 0 \quad 0.00$

$1: \mathrm{ASP}-31: \mathrm{HN}$

$1000.0 \overline{0} 0 \quad 0.00$

$1: \mathrm{ASP}-31: \mathrm{HN}$

$1000.0 \overline{0} 0 \quad 0.00$

1:ASP- $31: \mathrm{HN}$

$1000.0 \overline{0} 0.00$

$1: \mathrm{ASP}-31: \mathrm{HN}$

$1000.0 \overline{0} 0 \quad 0.00$

$1: \mathrm{ASP}-31: \mathrm{HN}$

$1000.0 \overline{0} 0 \quad 0.00$

$1: \mathrm{ASP}-31: \mathrm{HN}$

$1000.0 \overline{0} 0 \quad 0.00$

$1: \mathrm{ASP}-31: \mathrm{HN}$

$1000.0 \overline{0} 0.00$

$1: \mathrm{ASP}-31: \mathrm{HN}$

$1000.0 \overline{0} 0 \quad 0.00$

1:ASP- $31: \mathrm{HN}$

$1000.0 \overline{0} 0 \quad 0.00$

$1:$ LEU $32: \mathrm{HA}$

$1000 . \overline{0} 00 \quad 0.00$

$1: \mathrm{LEU} 32$ : HA

$1000 . \overline{0} 00 \quad 0.00$

$1: \mathrm{LEU} 32: \mathrm{HA}$

$1000 . \overline{0} 00 \quad 0.00$

$1:$ LEU 32 : HA

$1000 . \overline{0} 00 \quad 0.00$

$1:$ LEU_32: HN

$1000 . \overline{0} 00 \quad 0.00$

$1:$ LEU_32:HN

$1000 . \overline{0} 00 \quad 0.00$

1 : LEU $32: \mathrm{HN}$

$1000 . \overline{0} 00 \quad 0.00$

\begin{tabular}{|c|c|c|c|c|c|}
\hline $1:$ CYS_28:HB* & -1.000 & 6.000 & 5.000 & 10.00 & 10.00 \\
\hline $1: V A L \_33: H B$ & -1.000 & 3.400 & 3.000 & 10.00 & 10.00 \\
\hline $1:$ ALA_29:HB* & -1.000 & 6.000 & 5.000 & 10.00 & 10.00 \\
\hline $1:$ ALA_29:HA & -1.000 & 5.000 & 3.000 & 10.00 & 10.00 \\
\hline $1: \mathrm{GLU}-\_34: \mathrm{HB}$ * & -1.000 & 4.400 & 3.000 & 10.00 & 10.00 \\
\hline $1:$ LEU_27:HB* & -1.000 & 7.000 & 5.000 & 10.00 & 10.00 \\
\hline $1:$ LEU_27:HD1* & -1.000 & 5.400 & 3.000 & 10.00 & 10.00 \\
\hline $1:$ LEU_27:HD2* & -1.000 & 5.400 & 3.000 & 10.00 & 10.00 \\
\hline $1:$ LEU_27:HA & -1.000 & 5.000 & 3.000 & 10.00 & 10.00 \\
\hline $1:$ CYS_28:HB* & -1.000 & 6.000 & 5.000 & 10.00 & 10.00 \\
\hline $1:$ CYS_28:HN & -1.000 & 5.000 & 3.000 & 10.00 & 10.00 \\
\hline $1:$ ALA_29:HA & -1.000 & 4.300 & 3.000 & 10.00 & 10.00 \\
\hline $1:$ ALA_29:HB* & -1.000 & 6.000 & 5.000 & 10.00 & 10.00 \\
\hline $1: \mathrm{SER} \_30: \mathrm{HB} *$ & -1.000 & 4.400 & 3.000 & 10.00 & 10.00 \\
\hline $1:$ LEU_32:HA & -1.000 & 5.000 & 3.000 & 10.00 & 10.00 \\
\hline $1:$ LEU_32:HB* & -1.000 & 6.000 & 5.000 & 10.00 & 10.00 \\
\hline $1: \mathrm{LEU}_{-} 32: \mathrm{HD} 1$ * & -1.000 & 5.500 & 3.000 & 10.00 & 10.00 \\
\hline $1:$ LEU_32:HD2 * & -1.000 & 5.500 & 3.000 & 10.00 & 10.00 \\
\hline $1: L E U_{-} 32: \mathrm{HN}$ & -1.000 & 2.700 & 2.700 & 10.00 & 10.00 \\
\hline $1: \mathrm{VAL} \_33: \mathrm{HN}$ & -1.000 & 5.000 & 3.000 & 10.00 & 10.00 \\
\hline $1: \mathrm{GLU}-\_34: \mathrm{HB}$ * & -1.000 & 5.300 & 3.000 & 10.00 & 10.00 \\
\hline $1: L E U \_27: H A$ & -1.000 & 5.500 & 3.000 & 10.00 & 10.00 \\
\hline $1:$ ALA_35:HB* & -1.000 & 3.700 & 3.000 & 10.00 & 10.00 \\
\hline $1: \mathrm{LEU}_{-} 36: \mathrm{HD} 1$ * & -1.000 & 6.000 & 5.000 & 10.00 & 10.00 \\
\hline $1: \mathrm{LEU}_{-} 36: \mathrm{HD} 2$ * & -1.000 & 6.000 & 5.000 & 10.00 & 10.00 \\
\hline $1:$ ALA_29:HB* & -1.000 & 6.000 & 5.000 & 10.00 & 10.00 \\
\hline $1: \mathrm{SER} \_30: \mathrm{HA}$ & -1.000 & 5.000 & 3.000 & 10.00 & 10.00 \\
\hline 1:SER $30: \mathrm{HB} *$ & -1.000 & 6.000 & 5.000 & 10.00 & 10.00 \\
\hline
\end{tabular}




\begin{tabular}{|c|c|c|c|c|c|c|}
\hline 1:LEU_32:HN & $1: \mathrm{ASP}-\_31: \mathrm{HA}$ & -1.000 & 3.400 & 3.000 & 10.00 & 10.00 \\
\hline $\begin{array}{l}1000.000 \quad 0.00 \\
1: \mathrm{LEU} 32: \mathrm{HN}\end{array}$ & $1: \mathrm{GLU}-34: \mathrm{HA}$ & -1.000 & 5.500 & 3.000 & 10.00 & 10.00 \\
\hline $000 . \overline{0} 00 \quad 0.00$ & & & & & & \\
\hline VAL_33:HA & $1:$ LEU_36:HB* & -1.000 & 3.700 & 3.000 & 10.00 & 10.00 \\
\hline $000 . \overline{0} 00 \quad 0.00$ & & & & & & \\
\hline :VAL_33:HN & $1:$ ALA_29:HB* & -1.000 & 6.000 & 5.000 & 10.00 & 10.00 \\
\hline $000 . \overline{0} 00 \quad 0.00$ & & & & & & \\
\hline $\begin{array}{l}: \mathrm{VAL} 33: \mathrm{HN} \\
000.000 \quad 0.00\end{array}$ & $1: \mathrm{SER} \_30: \mathrm{HA}$ & -1.000 & 5.000 & 3.000 & 10.00 & 10.00 \\
\hline :VAL_33:HN & $1:$ ASP-_31:HA & -1.000 & 5.000 & 3.000 & 10.00 & 10.00 \\
\hline $000 . \overline{0} 00 \quad 0.00$ & & & & & & \\
\hline $\begin{array}{l}: \text { VAL } 33: \text { HN } \\
000.000 \quad 0.00\end{array}$ & $1: \mathrm{ASP}-\_31: \mathrm{HB}$ * & -1.000 & 6.000 & 5.000 & 10.00 & 10.00 \\
\hline $\begin{array}{l}: \mathrm{VAL} 33: \mathrm{HN} \\
000 . \overline{0} 00 \quad 0.00\end{array}$ & $1:$ LEU_32:HA & -1.000 & 5.000 & 3.000 & 10.00 & 10.00 \\
\hline :VAL_33:HN & $1:$ LEU_32:HB* & -1.000 & 6.000 & 5.000 & 10.00 & 10.00 \\
\hline $000 . \overline{0} 00 \quad 0.00$ & & & & & & \\
\hline $\begin{array}{l}: \text { VAL_33:HN } \\
000 . \overline{0} 00 \quad 0.00\end{array}$ & $1: \mathrm{GLU}-\_34: \mathrm{HN}$ & -1.000 & 3.400 & 3.000 & 10.00 & 10.00 \\
\hline $\begin{array}{l}: \mathrm{GLU}-34: \mathrm{HA} \\
000.000 \quad 0.00\end{array}$ & $1:$ TYR_37:HB* & -1.000 & 3.700 & 3.000 & 10.00 & 10.00 \\
\hline $\begin{array}{l}: \text { GLU- } 34: \mathrm{HN} \\
000.000 \quad 0.00\end{array}$ & $1:$ VAL_33:HA & -1.000 & 3.400 & 3.000 & 10.00 & 10.00 \\
\hline $\begin{array}{l}: \mathrm{GLU}-34: \mathrm{HN} \\
000.0 \overline{0} 0.00\end{array}$ & $1:$ VAL_33:HGI * & -1.000 & 4.400 & 3.000 & 10.00 & 10.00 \\
\hline $\begin{array}{l}: \text { GLU- } 34: \mathrm{HN} \\
000.0 \overline{0} 0.00\end{array}$ & $1:$ VAL_33:HG2 * & -1.000 & 6.000 & 5.000 & 10.00 & 10.00 \\
\hline $\begin{array}{l}: \text { GLU- } 34: \mathrm{HN} \\
000.000 \quad 0.00\end{array}$ & $1:$ ALA_35:HB* & -1.000 & 6.000 & 5.000 & 10.00 & 10.00 \\
\hline $\begin{array}{l}1: \mathrm{GLU}-34: \mathrm{HN} \\
1000.000 \quad 0.00\end{array}$ & $1:$ ALA_35:HN & -1.000 & 2.700 & 2.700 & 10.00 & 10.00 \\
\hline $\begin{array}{l}1: \text { ALA_35:HA } \\
1000.000 \quad 0.00\end{array}$ & $1:$ GLU-_34:HA & -1.000 & 5.000 & 3.000 & 10.00 & 10.00 \\
\hline $\begin{array}{l}1: \text { ALA_35:HA } \\
1000 . \overline{0} 00 \quad 0.00\end{array}$ & $1:$ LEU_38:HA & -1.000 & 5.000 & 3.000 & 10.00 & 10.00 \\
\hline $\begin{array}{l}1: \text { ALA_35:HA } \\
1000.000 \quad 0.00\end{array}$ & $1:$ LEU_38:HB* & -1.000 & 6.000 & 5.000 & 10.00 & 10.00 \\
\hline $\begin{array}{l}1: \text { ALA } 35: \text { HN } \\
1000.000 \quad 0.00\end{array}$ & $1: \mathrm{ASP}-{ }^{2} 31: \mathrm{HA}$ & -1.000 & 5.000 & 3.000 & 10.00 & 10.00 \\
\hline $\begin{array}{l}1: \text { ALA } 35: \text { HN } \\
1000 . \overline{0} 00 \quad 0.00\end{array}$ & $1:$ LEU_32:HA & -1.000 & 5.000 & 3.000 & 10.00 & 10.00 \\
\hline $\begin{array}{l}1: \text { ALA } 35: \text { HN } \\
1000.000 \quad 0.00\end{array}$ & $1:$ VAL_33:HA & -1.000 & 5.000 & 3.000 & 10.00 & 10.00 \\
\hline $\begin{array}{l}1: \text { ALA_35:HN } \\
1000 . \overline{0} 00 \quad 0.00\end{array}$ & $1: \mathrm{GLU}-\_34: \mathrm{HA}$ & -1.000 & 3.400 & 3.000 & 10.00 & 10.00 \\
\hline $\begin{array}{l}1: \text { ALA } 35: \text { HN } \\
1000.0000 .00\end{array}$ & $1: \mathrm{GLU}-{ }_{-} 34: \mathrm{HB}^{*}$ & -1.000 & 3.700 & 3.000 & 10.00 & 10.00 \\
\hline $\begin{array}{l}1: \text { ALA } 35: \text { HN } \\
1000.000 \quad 0.00\end{array}$ & $1:$ LEU_36:HN & -1.000 & 2.700 & 2.700 & 10.00 & 10.00 \\
\hline $\begin{array}{l}1: \mathrm{LEU} 36: \mathrm{HA} \\
1000 . \overline{0} 00 \quad 0.00\end{array}$ & $1:$ VAL_39:HGI* & -1.000 & 6.000 & 5.000 & 10.00 & 10.00 \\
\hline $\begin{array}{l}1: \text { LEU } 36: \mathrm{HA} \\
1000.000 \quad 0.00\end{array}$ & $1:$ VAL_39:HG2* & -1.000 & 6.000 & 5.000 & 10.00 & 10.00 \\
\hline $\begin{array}{l}1: \mathrm{LEU} 36: \mathrm{HN} \\
1000 . \overline{0} 00 \quad 0.00\end{array}$ & $1:$ LEU_32:HA & -1.000 & 3.400 & 3.000 & 10.00 & 10.00 \\
\hline
\end{tabular}




\begin{tabular}{|c|c|c|c|c|c|c|}
\hline $1:$ LEU_36:HN & $1:$ LEU_32:HD1* & -1.000 & 6.500 & 5.500 & 10.00 & 10.00 \\
\hline $1000 . \overline{0} 00 \quad 0.00$ & & & & & & \\
\hline $\begin{array}{l}1: \mathrm{LEU} 36: \mathrm{HN} \\
1000.0000 .00\end{array}$ & $1: \mathrm{VAL} \_33: \mathrm{HA}$ & -1.000 & 3.700 & 3.000 & 10.00 & 10.00 \\
\hline :LEU $36: \mathrm{HN}$ & $1:$ ALA_35:HA & -1.000 & 4.300 & 3.000 & 10.00 & 10.00 \\
\hline $\begin{array}{l}000.000 \quad 0.00 \\
: \mathrm{LEU} \quad 36: \mathrm{HN}\end{array}$ & 1:ALA 35:HB* & -1.000 & 3.700 & 3.000 & 10.00 & 10.00 \\
\hline $000 . \overline{0} 00 \quad 0.00$ & 1.10H_- & +.000 & . 10 & & & \\
\hline $\begin{array}{l}: \text { LEU } 36: \mathrm{HN} \\
000.000 \quad 0.00\end{array}$ & 1:TYR_37:HA & -1.000 & 5.500 & 3.000 & 10.00 & 10.00 \\
\hline $\begin{array}{l}: \text { LEU_36:HN } \\
000 . \overline{0} 00 \quad 0.00\end{array}$ & $1:$ TYR_37:HN & -1.000 & 2.700 & 2.700 & 10.00 & 10.00 \\
\hline $\begin{array}{l}: \text { TYR } 37: \mathrm{HB}^{*} \\
000.000 \quad 0.00\end{array}$ & $1: \mathrm{VAL} \_33: \mathrm{HA}$ & -1.000 & 6.000 & 5.000 & 10.00 & 10.00 \\
\hline $\begin{array}{l}: \text { TYR_37:HD* } \\
000.000 \quad 0.00\end{array}$ & $1:$ VAL_33:HGI* & -1.000 & 6.400 & 3.400 & 10.00 & 10.00 \\
\hline $\begin{array}{l}=\text { TYR_37:HD* } \\
000 . \overline{0} 00 \quad 0.00\end{array}$ & $1: \mathrm{VAL} \_33: \mathrm{HG} 2$ * & -1.000 & 6.400 & 3.400 & 10.00 & 10.00 \\
\hline $\begin{array}{l}: \text { TYR } 37: \text { HD* }^{*} \\
000.000 \quad 0.00\end{array}$ & $1:$ LEU_38:HA & -1.000 & 6.000 & 3.000 & 10.00 & 10.00 \\
\hline $\begin{array}{l}1: \text { TYR_37: } \mathrm{HE}^{\star} \\
1000.000 \quad 0.00\end{array}$ & 1:VAL_33:HGI* & -1.000 & 6.400 & 3.000 & 10.00 & 10.00 \\
\hline $\begin{array}{l}1: \text { TYR_37: } \mathrm{HE}^{*} \\
1000 . \overline{0} 00 \quad 0.00\end{array}$ & $1: V A L \_33: H G 2 *$ & -1.000 & 6.400 & 3.000 & 10.00 & 10.00 \\
\hline $\begin{array}{l}1: \text { TYR_37:HN } \\
1000.000 \quad 0.00\end{array}$ & $1: \mathrm{GLU}-\_34: \mathrm{HA}$ & -1.000 & 3.400 & 3.000 & 10.00 & 10.00 \\
\hline $\begin{array}{l}1: \text { TYR } 37: \text { HN } \\
1000.000 \quad 0.00\end{array}$ & $1:$ ALA_35:HB* & -1.000 & 6.000 & 5.000 & 10.00 & 10.00 \\
\hline $\begin{array}{l}1: \text { TYR_37:HN } \\
1000 . \overline{0} 00 \quad 0.00\end{array}$ & $1:$ LEU_36:HA & -1.000 & 5.000 & 3.000 & 10.00 & 10.00 \\
\hline $\begin{array}{l}1: \text { TYR } 37: H N \\
1000.000 \quad 0.00\end{array}$ & $1:$ LEU_36:HB* & -1.000 & 4.400 & 3.000 & 10.00 & 10.00 \\
\hline $\begin{array}{l}1: \text { TYR } 37: H N \\
1000.000 \quad 0.00\end{array}$ & $1:$ LEU_36:HDI* & -1.000 & 6.000 & 5.000 & 10.00 & 10.00 \\
\hline $\begin{array}{l}1: \text { TYR_37:HN } \\
1000 . \overline{0} 00 \quad 0.00\end{array}$ & $1:$ LEU_36:HD2* & -1.000 & 6.000 & 5.000 & 10.00 & 10.00 \\
\hline $\begin{array}{l}1: \text { TYR } 37: \text { HN } \\
1000.000 \quad 0.00\end{array}$ & $1:$ LEU_36:HG & -1.000 & 5.000 & 3.000 & 10.00 & 10.00 \\
\hline $\begin{array}{l}1: \text { TYR_37:HN } \\
1000.000 \quad 0.00\end{array}$ & $1:$ LEU_38:HB* & -1.000 & 6.000 & 5.000 & 10.00 & 10.00 \\
\hline $\begin{array}{l}1: \text { TYR_37:HN } \\
1000.000 \quad 0.00\end{array}$ & $1:$ LEU_38:HN & -1.000 & 2.700 & 2.700 & 10.00 & 10.00 \\
\hline $\begin{array}{l}1: \text { TYR } 37: \text { HN } \\
1000.000 \quad 0.00\end{array}$ & $1:$ VAL_39:HN & -1.000 & 5.000 & 3.000 & 10.00 & 10.00 \\
\hline $\begin{array}{l}1: \mathrm{LEU} 38: \mathrm{HN} \\
1000.000 \quad 0.00\end{array}$ & 1:TYR_37:HA & -1.000 & 3.400 & 3.000 & 10.00 & 10.00 \\
\hline $\begin{array}{l}1: \mathrm{VAL} 39: \mathrm{HN} \\
1000.000 \quad 0.00\end{array}$ & $1:$ ALA_35:HB* & -1.000 & 6.000 & 5.000 & 10.00 & 10.00 \\
\hline $\begin{array}{l}1: \mathrm{VAL} 39: \mathrm{HN} \\
1000.000 \quad 0.00\end{array}$ & $1:$ LEU_36:HA & -1.000 & 3.400 & 3.000 & 10.00 & 10.00 \\
\hline $\begin{array}{l}1: \mathrm{VAL} 39: \mathrm{HN} \\
1000.000 \quad 0.00\end{array}$ & 1:TYR_37:HB* & -1.000 & 6.000 & 5.000 & 10.00 & 10.00 \\
\hline $\begin{array}{l}1: \mathrm{VAL} 39: \mathrm{HN} \\
1000.000 \quad 0.00\end{array}$ & $1:$ LEU_38:HA & -1.000 & 3.400 & 3.000 & 10.00 & 10.00 \\
\hline $\begin{array}{l}1: \mathrm{VAL} 39: \mathrm{HN} \\
1000 . \overline{0} 00 \quad 0.00\end{array}$ & $1:$ LEU_38:HB* & -1.000 & 4.400 & 3.000 & 10.00 & 10.00 \\
\hline
\end{tabular}


1:VAL $39: \mathrm{HN}$

$1000 . \overline{0} 00 \quad 0.00$

$1: \mathrm{VAL} \quad 39: \mathrm{HN}$

$1000 . \overline{0} 00 \quad 0.00$

1 :VAL $39: \mathrm{HN}$

$1000.000 \quad 0.00$

1 : CYS $40: \mathrm{HA}$

$1000 . \overline{0} 00 \quad 0.00$

1 :CYS $40: \mathrm{HA}$

$1000 . \overline{0} 00 \quad 0.00$

1 :CYS $40: \mathrm{HN}$

$1000 . \overline{0} 00 \quad 0.00$

$1:$ CYS $40: \mathrm{HN}$

$1000 . \overline{0} 00 \quad 0.00$

$1:$ CYS $40: \mathrm{HN}$

$1000 . \overline{0} 00 \quad 0.00$

$1:$ CYS $40: \mathrm{HN}$

$1000 . \overline{0} 00 \quad 0.00$

$1:$ CYS $40: \mathrm{HN}$

$1000 . \overline{0} 00 \quad 0.00$

$1:$ CYS $40: \mathrm{HN}$

$1000 . \overline{0} 00 \quad 0.00$

1 :CYS $40: \mathrm{HN}$

$1000 . \overline{0} 00 \quad 0.00$

1 :CYS $40: \mathrm{HN}$

$1000 . \overline{0} 00 \quad 0.00$

1 :CYS $40: \mathrm{HN}$

$1000 . \overline{0} 00 \quad 0.00$

$1:$ CYS $40: \mathrm{HN}$

$1000 . \overline{0} 00 \quad 0.00$

$1:$ CYS $40: \mathrm{HN}$

$1000 . \overline{0} 00 \quad 0.00$

1 :CYS $40: \mathrm{HN}$

$1000 . \overline{0} 00 \quad 0.00$

1 :GLY_41:HA*

$1000 . \overline{0} 00 \quad 0.00$

$1: \mathrm{GLY} 41: \mathrm{HN}$

$1000 . \overline{0} 00 \quad 0.00$

$1:$ GLY $41: \mathrm{HN}$

$1000 . \overline{0} 00 \quad 0.00$

$1:$ GLY $41: \mathrm{HN}$

$1000 . \overline{0} 00 \quad 0.00$

1 :GLY 41 : HN

$1000 . \overline{0} 00 \quad 0.00$

$1:$ GLY $41: \mathrm{HN}$

$1000 . \overline{0} 00 \quad 0.00$

$1:$ GLU- 42 : HN

$1000.0 \overline{0} 0 \quad 0.00$

$1: \mathrm{GLU}-42: \mathrm{HN}$

$1000.0 \overline{0} 0 \quad 0.00$

$1: \mathrm{ARG}+43: \mathrm{HN}$

$1000.0 \overline{0} 0 \quad 0.00$

$1: \mathrm{ARG}+43: \mathrm{HN}$

$1000.0 \overline{0} 0 \quad 0.00$

$1: \mathrm{ARG}+43: \mathrm{HN}$

$1000.0 \overline{0} 0 \quad 0.00$

\begin{tabular}{|c|c|c|c|c|c|}
\hline $1:$ LEU_38:HN & -1.000 & 3.400 & 3.000 & 10.00 & 10.00 \\
\hline $1:$ CYS_4 $40: \mathrm{HN}$ & -1.000 & 2.700 & 2.700 & 10.00 & 10.00 \\
\hline $1: \mathrm{GLY} \_41: \mathrm{HN}$ & -1.000 & 5.000 & 3.000 & 10.00 & 10.00 \\
\hline $1:$ VAL_39:HGI* & -1.000 & 4.400 & 3.000 & 10.00 & 10.00 \\
\hline $1:$ VAL_39:HG2* & -1.000 & 5.300 & 3.000 & 10.00 & 10.00 \\
\hline $1:$ LEU_36:HA & -1.000 & 3.400 & 3.000 & 10.00 & 10.00 \\
\hline $1: \mathrm{LEU} \_36: \mathrm{HD} 1$ * & -1.000 & 6.000 & 5.000 & 10.00 & 10.00 \\
\hline $1:$ LEU_36:HD2* & -1.000 & 6.000 & 5.000 & 10.00 & 10.00 \\
\hline $1:$ TYR_37:HA & -1.000 & 5.000 & 3.000 & 10.00 & 10.00 \\
\hline $1:$ LEU_38:HA & -1.000 & 5.000 & 3.000 & 10.00 & 10.00 \\
\hline $1:$ LEU_38:HB* & -1.000 & 6.000 & 5.000 & 10.00 & 10.00 \\
\hline $1: \mathrm{VAL} 339: \mathrm{HA}$ & -1.000 & 4.300 & 3.000 & 10.00 & 10.00 \\
\hline $1: \mathrm{VAL} 339: \mathrm{HB}$ & -1.000 & 4.300 & 3.000 & 10.00 & 10.00 \\
\hline $1:$ VAL_39:HGI* & -1.000 & 6.000 & 5.000 & 10.00 & 10.00 \\
\hline 1:VAL_39:HG2* & -1.000 & 6.000 & 5.000 & 10.00 & 10.00 \\
\hline $1: \mathrm{GLY} \_41: \mathrm{HA}^{*}$ & -1.000 & 6.000 & 5.000 & 10.00 & 10.00 \\
\hline $1: \mathrm{GLY} \_41: \mathrm{HN}$ & -1.000 & 2.700 & 2.700 & 10.00 & 10.00 \\
\hline $1: \mathrm{GLU}-\_42: \mathrm{HB} *$ & -1.000 & 5.400 & 3.000 & 10.00 & 10.00 \\
\hline $1:$ TYR_37:HA & -1.000 & 5.000 & 3.000 & 10.00 & 10.00 \\
\hline $1:$ LEU_38 $: \mathrm{HA}$ & -1.000 & 5.000 & 3.000 & 10.00 & 10.00 \\
\hline $1: \mathrm{VAL} \_39: \mathrm{HA}$ & -1.000 & 5.000 & 3.000 & 10.00 & 10.00 \\
\hline $1:$ CYS_4 $40: \mathrm{HA}$ & -1.000 & 3.400 & 3.000 & 10.00 & 10.00 \\
\hline $1:$ CYS_4 $40: \mathrm{HB}^{*}$ & -1.000 & 4.400 & 3.000 & 10.00 & 10.00 \\
\hline $1:$ GLY_41:HA* & -1.000 & 6.000 & 5.000 & 10.00 & 10.00 \\
\hline $1:$ ARG+_43:HN & -1.000 & 3.400 & 3.000 & 10.00 & 10.00 \\
\hline $1:$ CYS_4 $40: \mathrm{HB}^{*}$ & -1.000 & 6.000 & 5.000 & 10.00 & 10.00 \\
\hline $1:$ GLY_41:HA* & -1.000 & 6.000 & 5.000 & 10.00 & 10.00 \\
\hline $1:$ GLU-_42:HG* & -1.000 & 6.000 & 5.000 & 10.00 & 10.00 \\
\hline
\end{tabular}




\begin{tabular}{|c|c|c|c|c|c|c|}
\hline $1: \mathrm{ARG}+43: \mathrm{HN}$ & $1: \mathrm{GLY}_{-} 44: \mathrm{HN}$ & -1.000 & 2.700 & 2.700 & 10.00 & 10.00 \\
\hline $1000.0 \overline{0} 0 \quad 0.00$ & & & & & & \\
\hline 1:GLY $44:$ HN & $1:$ ARG+_43:HA & -1.000 & 3.400 & 3.000 & 10.00 & 10.00 \\
\hline$:$ GLY $44: \mathrm{HN}$ & $1: \mathrm{GLU}-\_42: \mathrm{HA}$ & -1.000 & 3.400 & 3.000 & 10.00 & 10.00 \\
\hline $\begin{array}{l}000.000 \quad 0.00 \\
: G L Y \quad 44: H N\end{array}$ & $1: \mathrm{ARG}+43: \mathrm{HB} *$ & -1.000 & 3.700 & 3.000 & 10.00 & 10.00 \\
\hline $000 . \overline{0} 00 \quad 0.00$ & & & & & & \\
\hline $\begin{array}{l}: \text { PHE } 45: \mathrm{HE}^{\star} \\
000.000 \quad 0.00\end{array}$ & $1:$ VAL_33:HGI * & -1.000 & 6.400 & 3.400 & 10.00 & 10.00 \\
\hline$: \mathrm{PHE}_{-} 45: \mathrm{HE}^{\star}$ & $1:$ VAL_33:HG2* & -1.000 & 6.400 & 3.400 & 10.00 & 10.00 \\
\hline $000 . \overline{0} 00 \quad 0.00$ & & & & & & \\
\hline : $\mathrm{PHE} 45: \mathrm{HE}^{\star}$ & $1:$ LEU_36:HDI* & -1.000 & 8.000 & 5.000 & 10.00 & 10.00 \\
\hline $000 . \overline{0} 00 \quad 0.00$ & & & & & & \\
\hline $\begin{array}{l}: \text { PHE } 45: \mathrm{HE}^{\star} \\
000.000 \quad 0.00\end{array}$ & $1:$ LEU_36:HD2* & -1.000 & 8.000 & 5.000 & 10.00 & 10.00 \\
\hline$: \mathrm{PHE} \quad 45: \mathrm{HN}$ & $1: \mathrm{GLY}_{-} 44: \mathrm{HA}^{*}$ & -1.000 & 4.400 & 3.000 & 10.00 & 10.00 \\
\hline $\begin{array}{l}000.000 \quad 0.00 \\
: \text { PHE } 46: \mathrm{HA} \\
000.000 \quad 0.00\end{array}$ & $1:$ TYR_47:HB* & -1.000 & 6.000 & 5.000 & 10.00 & 10.00 \\
\hline $\begin{array}{l}: \mathrm{PHE} \quad 46: \mathrm{HN} \\
000 . \overline{0} 00 \quad 0.00\end{array}$ & $1:$ CYS_20:HA & -1.000 & 5.000 & 3.000 & 10.00 & 10.00 \\
\hline $\begin{array}{l}1: \mathrm{PHE} \quad 46: \mathrm{HN} \\
1000.000 \quad 0.00\end{array}$ & $1:$ PHE_ $45: \mathrm{HA}$ & -1.000 & 2.700 & 2.700 & 10.00 & 10.00 \\
\hline $\begin{array}{l}1: \mathrm{PHE} \quad 46: \mathrm{HN} \\
1000.000 \quad 0.00\end{array}$ & $1:$ PHE_ $45: \mathrm{HB}^{*}$ & -1.000 & 4.400 & 3.000 & 10.00 & 10.00 \\
\hline $\begin{array}{l}1: \mathrm{PHE} 46: \mathrm{HN} \\
1000 . \overline{0} 00 \quad 0.00\end{array}$ & $1:$ PHE_4 $5: \mathrm{HD}^{*}$ & -1.000 & 7.000 & 5.000 & 10.00 & 10.00 \\
\hline $\begin{array}{l}1: \text { TYR_4 } 7: \text { HD* }^{*} \\
1000 . \overline{0} 00 \quad 0.00\end{array}$ & 1:ILE_2:HG2* & -1.000 & 5.700 & 3.000 & 10.00 & 10.00 \\
\hline $\begin{array}{l}1: \text { TYR_47:HD* } \\
1000 . \overline{000} 0.00\end{array}$ & $1:$ LEU_32:HG & -1.000 & 5.400 & 3.000 & 10.00 & 10.00 \\
\hline $\begin{array}{l}1: \text { TYR_47:HD* } \\
1000.000 \quad 0.00\end{array}$ & $1:$ VAL_33:HGI* & -1.000 & 6.400 & 3.400 & 10.00 & 10.00 \\
\hline $\begin{array}{l}1: \text { TYR_47:HD* } \\
1000 . \overline{000} 0.00\end{array}$ & $1:$ VAL_33:HG2* & -1.000 & 6.400 & 3.400 & 10.00 & 10.00 \\
\hline $\begin{array}{l}1: \text { TYR_47:HD* } \\
1000.000 \quad 0.00\end{array}$ & $1:$ LEU_36:HDI* & -1.000 & 6.400 & 3.400 & 10.00 & 10.00 \\
\hline $\begin{array}{l}1: \text { TYR_47:HD* } \\
1000 . \overline{000} 0.00\end{array}$ & $1: \mathrm{LEU} \_36: \mathrm{HD} 2 *$ & -1.000 & 6.400 & 3.400 & 10.00 & 10.00 \\
\hline $\begin{array}{l}1: \text { TYR_47:HD* } \\
1000 . \overline{000} 0.00\end{array}$ & $1:$ THR_4 $8: H B$ & -1.000 & 7.000 & 5.000 & 10.00 & 10.00 \\
\hline $\begin{array}{l}1: \text { TYR_47: } \mathrm{HE}^{*} \\
1000.000 \quad 0.00\end{array}$ & 1:ILE_2:HG2* & -1.000 & 5.700 & 3.000 & 10.00 & 10.00 \\
\hline $\begin{array}{l}1: \text { TYR_47: } \text { HE* }^{*} \\
1000.000 \quad 0.00\end{array}$ & $1:$ VAL_3:HG1* & -1.000 & 6.400 & 3.400 & 10.00 & 10.00 \\
\hline $\begin{array}{l}1: \text { TYR_47: } \mathrm{HE}^{\star} \\
1000.000 \quad 0.00\end{array}$ & $1:$ VAL_3:HG2* & -1.000 & 6.400 & 3.400 & 10.00 & 10.00 \\
\hline $\begin{array}{l}1: \text { TYR_47: } \mathrm{HE}^{*} \\
1000.000 \quad 0.00\end{array}$ & $1:$ LEU_32:HD1* & -1.000 & 6.400 & 3.400 & 10.00 & 10.00 \\
\hline $\begin{array}{l}1: \text { TYR_47: } \mathrm{HE}^{\star} \\
1000.000 \quad 0.00\end{array}$ & $1:$ LEU_32:HD2 * & -1.000 & 6.400 & 3.400 & 10.00 & 10.00 \\
\hline $\begin{array}{l}1: \text { TYR_47: } \mathrm{HE}^{*} \\
1000.000 \quad 0.00\end{array}$ & $1:$ LEU_36:HD1 * & -1.000 & 8.000 & 5.000 & 10.00 & 10.00 \\
\hline $\begin{array}{l}1: \text { TYR_47: } \mathrm{HE}^{\star} \\
1000 . \overline{0} 00 \quad 0.00\end{array}$ & $1:$ LEU_36:HD2* & -1.000 & 8.000 & 5.000 & 10.00 & 10.00 \\
\hline
\end{tabular}


1:TYR $47: \mathrm{HE}^{*}$

$1000 . \overline{0} 00 \quad 0.00$

1:TYR 47 : $\mathrm{HE}^{*}$

$1000 . \overline{0} 00 \quad 0.00$

1:TYR $47: \mathrm{HN}$

$1000 . \overline{0} 00 \quad 0.00$

1:TYR $47: \mathrm{HN}$

$1000 . \overline{0} 00 \quad 0.00$

$1:$ TYR $47: \mathrm{HN}$

$1000 . \overline{0} 00 \quad 0.00$

$1:$ THR $48: \mathrm{HN}$

$1000 . \overline{0} 00 \quad 0.00$

$1:$ THR $48: \mathrm{HN}$

$1000 . \overline{0} 00 \quad 0.00$

$1: \mathrm{LYS}+49: \mathrm{HA}$

$1000.0 \overline{0} 0 \quad 0.00$

1:LYS + 49:HN

$1000.0 \overline{0} 0 \quad 0.00$

$1: \mathrm{LYS}+49: \mathrm{HN}$

$1000.0 \overline{0} 0 \quad 0.00$

$1:$ LYS $+49: \mathrm{HN}$

$1000.0 \overline{0} 00.00$

$1:$ PRO_ $50: \mathrm{HA}$

$1000 . \overline{0} 00 \quad 0.00$

$1: \mathrm{PRO} 50$ : HD*

$1000 . \overline{0} 00 \quad 0.00$

1 : $\mathrm{PRO} 50$ : HD*

$1000 . \overline{0} 00 \quad 0.00$

1:THRC $51: \mathrm{HN}$

$1000.0 \overline{0} 0 \quad 0.00$

1:THRC $51: \mathrm{HN}$

$1000.0 \overline{0} 0 \quad 0.00$

1:THRC $51: \mathrm{HN}$

$1000.0 \overline{0} 0.00$

1:THRC_51:HN

$1000.0 \overline{0} 0.00$

$1:$ THRC_51:HN

$1000.0 \overline{0} 0 \quad 0.00$

1:THRC $51: \mathrm{HN}$

$1000.0 \overline{0} 0 \quad 0.00$

$1: \mathrm{CYS} 6: \mathrm{HN}$

$1000 . \overline{0} 00 \quad 0.00$

$1: \mathrm{CYS} \quad 6: \mathrm{HN}$

$1000 . \overline{0} 00 \quad 0.00$

$1:$ CYS $6: \mathrm{HA}$

$1000 . \overline{0} 00 \quad 0.00$

1:CYS $6: \mathrm{HA}$

$1000 . \overline{0} 00 \quad 0.00$

1:TYR_19:HN

$1000 . \overline{000} 0.00$

1:TYR_19:HN

$1000 . \overline{0} 00 \quad 0.00$

1:TYR_19:HA

$1000 . \overline{0} 00 \quad 0.00$

1:TYR 19: HA

$1000 . \overline{0} 00 \quad 0.00$

\begin{tabular}{|c|c|c|c|c|c|}
\hline $1:$ THR_48:HG2* & -1.000 & 6.400 & 3.4001 & 10.001 & 10.00 \\
\hline $1:$ LYS+_49:HG* & -1.000 & 6.400 & 3.4001 & 10.001 & 10.00 \\
\hline $1: \mathrm{PHE} \quad 46: \mathrm{HA}$ & -1.000 & 2.700 & 2.7001 & 10.001 & 10.00 \\
\hline $1:$ PHE $46: \mathrm{HB}^{*}$ & -1.000 & 4.400 & 3.0001 & 10.001 & 10.00 \\
\hline $1:$ THR_48:HN & -1.000 & 4.300 & 3.0001 & 10.001 & 10.00 \\
\hline $1:$ TYR_4 $7: H A$ & -1.000 & 3.400 & 3.0001 & 10.001 & 10.00 \\
\hline 1:TYR_47:HB* & -1.000 & 6.000 & 5.0001 & 10.001 & 10.00 \\
\hline $1:$ PRO_50:HD* & -1.000 & 3.700 & 3.0001 & 10.001 & 10.00 \\
\hline $1:$ THR_48:HA & -1.000 & 2.700 & 2.7001 & 10.001 & 10.00 \\
\hline $1:$ THR_48:HB & -1.000 & 5.000 & 3.0001 & 10.001 & 10.00 \\
\hline $1:$ THR_48:HG2 * & -1.000 & 4.400 & 3.0001 & 10.001 & 10.00 \\
\hline $1:$ THRC_51:HG2* & -1.000 & 6.000 & 5.0001 & 10.001 & 10.00 \\
\hline $1:$ LYS $+449: \mathrm{HB}^{*}$ & -1.000 & 7.000 & 5.0001 & 10.001 & 10.00 \\
\hline $1:$ LYS+_49:HG* & -1.000 & 5.400 & 3.0001 & 10.001 & 10.00 \\
\hline $1:$ LYS $+449: \mathrm{HB} *$ & -1.000 & 6.000 & 5.0001 & 10.001 & 10.00 \\
\hline $1:$ LYS+_49:HG* & -1.000 & 6.000 & 5.0001 & 10.001 & 10.00 \\
\hline $1: \mathrm{PRO} 50: \mathrm{HA}$ & -1.000 & 3.400 & 3.0001 & 10.001 & 10.00 \\
\hline $1: \mathrm{PRO} 550: \mathrm{HB} *$ & -1.000 & 4.400 & 3.0001 & 10.001 & 10.00 \\
\hline $1: \mathrm{PRO} 500: \mathrm{HD}$ * & -1.000 & 6.000 & 5.0001 & 10.001 & 10.00 \\
\hline $1: \mathrm{PRO} 50: \mathrm{HG}^{*}$ & -1.000 & 3.700 & 3.0001 & 10.001 & 10.00 \\
\hline $1:$ CYS_6:HB1 & -1.000 & 3.700 & 3.0001 & 10.001 & 10.00 \\
\hline $1:$ CYS_6:HB2 & -1.000 & 2.700 & 2.7001 & 10.00 & 10.00 \\
\hline 1:CYS 6:HB1 & -1.000 & 2.700 & 2.7001 & 10.001 & 10.00 \\
\hline $1:$ CYS_6:HB2 & -1.000 & 3.400 & 2.7001 & 10.001 & 10.00 \\
\hline 1:TYR_19:HB1 & -1.000 & 3.700 & 3.000 & 010.0 & 010.00 \\
\hline $1:$ TYR_19:HB2 & -1.000 & 2.700 & 2.700 & 010.0 & 010.00 \\
\hline 1:TYR_19:HB1 & -1.000 & 2.700 & 2.700 & 010.0 & 010.00 \\
\hline 1:TYR_19:HB2 & -1.000 & 3.400 & 2.700 & 010.0 & 010.00 \\
\hline
\end{tabular}


$1:$ HIS 26:HN

$1000 . \overline{0} 00 \quad 0.00$

$1: \mathrm{HIS} 26: \mathrm{HN}$

$1000 . \overline{0} 00 \quad 0.00$

1:HIS_26:HA

$1000 . \overline{0} 00 \quad 0.00$

$1: \mathrm{HIS} 26: \mathrm{HA}$

$1000 . \overline{0} 00 \quad 0.00$

$1:$ LEU $27: \mathrm{HN}$

$1000 . \overline{0} 00 \quad 0.00$

$1:$ LEU $27: \mathrm{HN}$

$1000 . \overline{0} 00 \quad 0.00$

1 : LEU 27 : HA

$1000 . \overline{0} 00 \quad 0.00$

$1: \mathrm{LEU} 27: \mathrm{HA}$

$1000 . \overline{0} 00 \quad 0.00$

1:ASP- $31: \mathrm{HN}$

$1000.0 \overline{0} 00.00$

$1: \mathrm{ASP}-31: \mathrm{HN}$

$1000.0 \overline{0} 0 \quad 0.00$

$1: \mathrm{ASP}-31: \mathrm{HA}$

$1000.0 \overline{0} 0.00$

$1:$ ASP $-31: \mathrm{HA}$

$1000.0 \overline{0} 0 \quad 0.00$

$1:$ LEU_32:HN

$1000 . \overline{0} 00 \quad 0.00$

$1: \mathrm{LEU} 32: \mathrm{HN}$

$1000 . \overline{0} 00 \quad 0.00$

$1:$ LEU 32:HA

$1000 . \overline{0} 00 \quad 0.00$

$1: \mathrm{LEU} 32$ : HA

$1000 . \overline{0} 00 \quad 0.00$

$1: \mathrm{CYS} 40: \mathrm{HN}$

$1000 . \overline{0} 00 \quad 0.00$

$1: \mathrm{CYS} 40: \mathrm{HN}$

$1000 . \overline{0} 00 \quad 0.00$

$1:$ CYS $40: \mathrm{HA}$

$1000 . \overline{0} 00 \quad 0.00$

1 : CYS $40: \mathrm{HA}$

$1000 . \overline{0} 00 \quad 0.00$

$1: \mathrm{SER} 12: \mathrm{HN}$

$1000 . \overline{0} 00 \quad 0.00$

$1:$ SER $12: \mathrm{HN}$

$1000 . \overline{0} 00 \quad 0.00$

$1: \mathrm{SER} 12: \mathrm{HA}$

$1000 . \overline{0} 00 \quad 0.00$

$1: S E R \quad 12: H A$

$1000 . \overline{0} 00 \quad 0.00$

$1: \mathrm{PHE} \quad 45: \mathrm{HN}$

$1000 . \overline{0} 00 \quad 0.00$

$1: \mathrm{PHE} 45: \mathrm{HN}$

$1000 . \overline{0} 00 \quad 0.00$

$1: \mathrm{PHE} \quad 45: \mathrm{HA}$

$1000 . \overline{0} 00 \quad 0.00$

$1: \mathrm{PHE} \quad 45: \mathrm{HA}$

$1000 . \overline{0} 00 \quad 0.00$

\begin{tabular}{|c|c|c|c|c|c|}
\hline $1:$ HIS_26:HB1 & -1.000 & 3.700 & 3.000 & 10.00 & 10.00 \\
\hline $1:$ HIS_26:HB2 & -1.000 & 2.700 & 2.700 & 10.00 & 10.00 \\
\hline $1:$ HIS $26:$ HB1 & -1.000 & 2.700 & 2.700 & 10.00 & 10.00 \\
\hline $1:$ HIS_26:HB2 & -1.000 & 3.400 & 2.700 & 10.00 & 10.00 \\
\hline $1:$ LEU_27:HB1 & -1.000 & 3.700 & 3.000 & 10.00 & 10.00 \\
\hline $1:$ LEU_27:HB2 & -1.000 & 2.700 & 2.700 & 10.00 & 10.00 \\
\hline $1:$ LEU_27:HB1 & -1.000 & 2.700 & 2.700 & 10.00 & 10.00 \\
\hline $1:$ LEU_27:HB2 & -1.000 & 3.400 & 2.700 & 10.00 & 10.00 \\
\hline $1: \mathrm{ASP}-\_31: \mathrm{HB} 1$ & -1.000 & 3.700 & 3.000 & 10.00 & 10.00 \\
\hline $1: \mathrm{ASP}-\_31: \mathrm{HB} 2$ & -1.000 & 2.700 & 2.700 & 10.00 & 010.00 \\
\hline $1: \mathrm{ASP}-31: \mathrm{HB} 1$ & -1.000 & 2.700 & 2.700 & 10.00 & 10.00 \\
\hline $1: \mathrm{ASP}-\_31: \mathrm{HB} 2$ & -1.000 & 3.400 & 2.700 & 10.00 & 010.00 \\
\hline $1:$ LEU_32:HB1 & -1.000 & 3.700 & 3.000 & 10.00 & 10.00 \\
\hline $1:$ LEU_32: HB2 & -1.000 & 2.700 & 2.700 & 10.00 & 10.00 \\
\hline $1:$ LEU_32:HB1 & -1.000 & 2.700 & 2.700 & 10.00 & 10.00 \\
\hline $1:$ LEU_32:HB2 & -1.000 & 3.400 & 2.700 & 10.00 & 10.00 \\
\hline $1:$ :YS_4 $40:$ HB1 & -1.000 & 3.700 & 3.000 & 10.00 & 10.00 \\
\hline $1:$ CYS_4 $40:$ HB2 & -1.000 & 2.700 & 2.700 & 10.00 & 10.00 \\
\hline 1 :CYS_40:HB1 & -1.000 & 2.700 & 2.700 & 10.00 & 10.00 \\
\hline $1:$ CYS_40:HB2 & -1.000 & 3.400 & 2.700 & 10.00 & 10.00 \\
\hline $1:$ SER_12:HB1 & -1.000 & 3.700 & 3.000 & 10.00 & 10.00 \\
\hline $1:$ SER_12:HB2 & -1.000 & 2.700 & 2.700 & 10.00 & 10.00 \\
\hline $1:$ SER_12:HB1 & -1.000 & 2.700 & 2.700 & 10.00 & 10.00 \\
\hline $1:$ SER_12:HB2 & -1.000 & 3.400 & 2.700 & 10.00 & 10.00 \\
\hline $1:$ PHE_4 $5:$ HB1 & -1.000 & 3.400 & 2.700 & 10.00 & 10.00 \\
\hline $1:$ PHE $45:$ HB2 & -1.000 & 3.700 & 3.000 & 10.00 & 10.00 \\
\hline $1:$ PHE_4 $5:$ HB1 & -1.000 & 2.700 & 2.700 & 10.00 & 10.00 \\
\hline $1:$ PHE $45:$ HB2 & -1.000 & 2.700 & 2.700 & 10.00 & 10.00 \\
\hline
\end{tabular}




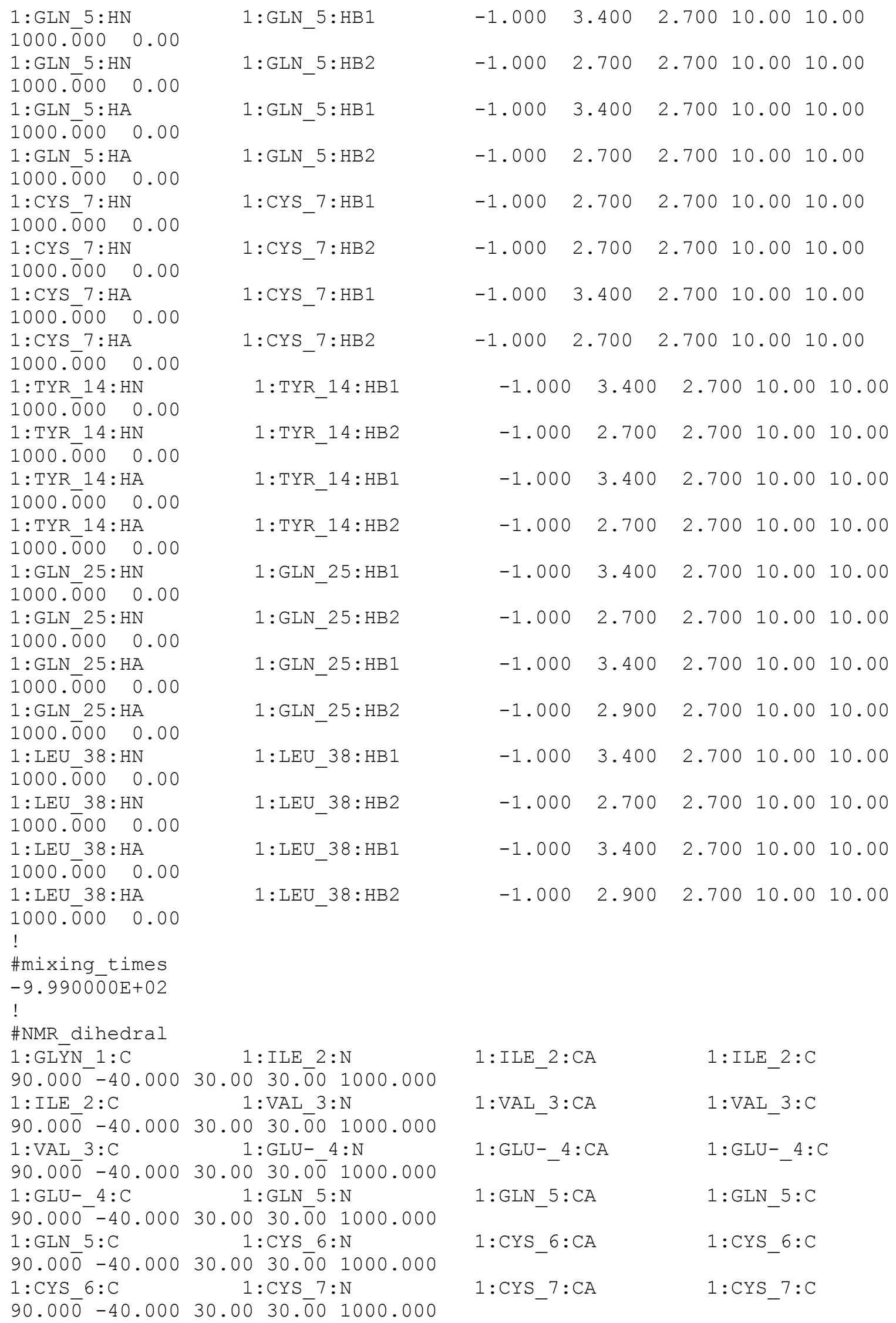


$1: \mathrm{SER} 12: \mathrm{C}$
$90.000-40$

$0.00030 .0030 . \overline{0} 0 \quad 1000.000$

$1:$ LEU_13:C $1:$ TYR_ $14: \mathrm{N}$

$90.00 \overline{0}-40.000 \quad 30.00 \quad 30 . \overline{0} 0 \quad 1000.000$

$1:$ TYR $14: \mathrm{C} \quad 1:$ GLN $15: \mathrm{N}$

$90.00 \overline{0}-40.000 \quad 30.00 \quad 30 . \overline{0} 0 \quad 1000.000$

$1:$ GLN $15: \mathrm{C} \quad 1:$ LEU $16: \mathrm{N}$

$90.00 \overline{0}-40.000 \quad 30.00 \quad 30 . \overline{0} 0 \quad 1000.000$

$1:$ LEU $16: \mathrm{C} \quad 1: \mathrm{GLU}-17: \mathrm{N}$

$90.00 \overline{0}-40.000 \quad 30.00 \quad 30.0 \overline{0} \quad 1000.000$

$1:$ GLU- $17: \mathrm{C} \quad 1: \mathrm{ASN} 18: \mathrm{N}$

$90.000^{-}-40.000 \quad 30.00 \quad 30.00 \quad 1000.000$

$1:$ ASN $18: \mathrm{C} \quad 1:$ TYR $19: \mathrm{N}$

$90.00 \overline{0}-40.000 \quad 30.00 \quad 30 . \overline{0} 0 \quad 1000.000$

$1:$ SER $30: \mathrm{C} \quad 1: \mathrm{ASP}-31: \mathrm{N}$

$90.00 \overline{0}-40.000 \quad 30.00 \quad 30.0 \overline{0} \quad 1000.000$

$1: A S P-31: C \quad 1: L E U ~ 32: N$

$90.000^{-}-40.000 \quad 30.00 \quad 30 . \overline{0} 0 \quad 1000.000$

$1:$ LEU 32:C $1:$ VAL $33: \mathrm{N}$

$90.00 \overline{0}-40.000 \quad 30.00 \quad 30 . \overline{0} 0 \quad 1000.000$

$1:$ VAL $33: \mathrm{C} \quad 1: \mathrm{GLU}-34: \mathrm{N}$

$90.00 \overline{0}-40.00030 .0030 .0 \overline{0} 1000.000$

$1: \mathrm{GLU}-34: \mathrm{C} \quad 1: \mathrm{ALA} \quad 35: \mathrm{N}$

$90.000^{-}-40.000 \quad 30.00 \quad 30 . \overline{0} 0 \quad 1000.000$

$1:$ ALA $35: \mathrm{C} \quad 1: \mathrm{LEU} \quad 36: \mathrm{N}$

$90.00 \overline{0}-40.000 \quad 30.00 \quad 30 . \overline{0} 0 \quad 1000.000$

$1:$ LEU $36: \mathrm{C} \quad 1:$ TYR $37: \mathrm{N}$

$90.00 \overline{0}-40.000 \quad 30.00 \quad 30 . \overline{0} 0 \quad 1000.000$

$1:$ TYR $37: \mathrm{C} \quad 1: \mathrm{LEU} 38: \mathrm{N}$

$90.00 \overline{0}-40.000 \quad 30.00 \quad 30 . \overline{0} 0 \quad 1000.000$

$1:$ LEU $38: C \quad 1:$ VAL $39: \mathrm{N}$

$90.00 \overline{0}-40.000 \quad 30.00 \quad 30 . \overline{0} 0 \quad 1000.000$

$1:$ VAL $39: \mathrm{C} \quad 1:$ CYS $40: \mathrm{N}$

$90.00 \overline{0}-40.000 \quad 30.00 \quad 30 . \overline{0} 0 \quad 1000.000$

$1:$ SER $9: C$

$1:$ ILE $10: \mathrm{N}$

$160.0 \overline{0} 0-80.000 \quad 30.00 \quad 30.00 \quad 1000.000$

$1:$ CYS $11: \mathrm{C} \quad 1:$ SER $12: \mathrm{N}$

$160.0 \overline{0} 0-80.000 \quad 30.00 \quad 30.00 \quad 1000.000$

$1:$ PHEN 22:C $1:$ VAL 23:N

$160.00 \overline{0}-80.000 \quad 30.00 \quad 30.00 \quad 1000.000$

$1:$ ASN $24: C$

$1:$ GLN $25: \mathrm{N}$

$160.0 \overline{0} 0-80.000 \quad 30.00 \quad 30.00 \quad 1000.000$

$1:$ HIS $26: \mathrm{C}$

$1:$ LEU $27: \mathrm{N}$

$160.0 \overline{0} 0-80.000 \quad 30.00 \quad 30.00 \quad 1000.000$

$1:$ PHE $45: \mathrm{C}$

$1:$ PHE $46: \mathrm{N}$

$160.0 \overline{0} 0 \quad-80.000 \quad 30.00 \quad 30.00 \quad 1000.000$

$1:$ TYR $47: \mathrm{C}$

$160.000-80.000 \quad 30.00 \quad 30.00 \quad 1000.000$

$1: \mathrm{GLU}-4: \mathrm{N}$

$100.00 \overline{0}-20.000 \quad 30.00 \quad 30 . \overline{0} 0 \quad 1000.000$

$1:$ ASN $18: \mathrm{N}$

$1: \mathrm{ASN} 18: \mathrm{CA}$

$100.0 \overline{0} 0-20.000 \quad 30.00 \quad 30.00 \quad 1000.000$

1:TYR_19:N

$1:$ TYR $19:$ CA

$100.0 \overline{0} 0-20.000 \quad 30.00 \quad 30.00 \quad 1000.000$

$1:$ CYS $20: \mathrm{N}$

$1:$ CYS $20: C A$

$100.0 \overline{0} 0-20.000 \quad 30.00 \quad 30.00 \quad 1000.000$

\begin{tabular}{|c|c|c|}
\hline $1:$ LEU_13:CA & $1:$ LEU_13:C & - \\
\hline $1:$ TYR_14:CA & $1:$ TYR_14:C & - \\
\hline $1:$ GLN_15:CA & $1: \mathrm{GLN}_{-} 15: \mathrm{C}$ & - \\
\hline $1:$ LEU_16:CA & $1:$ LEU_16:C & - \\
\hline $1: \mathrm{GLU}-\_17: \mathrm{CA}$ & $1:$ GLU___17:C & - \\
\hline $1: A S N \_18: C A$ & $1: \mathrm{ASN}_{-} 18: \mathrm{C}$ & - \\
\hline $1:$ TYR_19:CA & 1:TYR_19:C & - \\
\hline $1: \mathrm{ASP}-\_31: \mathrm{CA}$ & $1: \mathrm{ASP}-\_31: \mathrm{C}$ & - \\
\hline $1:$ LEU_32:CA & $1:$ LEU_32:C & - \\
\hline $1: \mathrm{VAL} \_33: \mathrm{CA}$ & $1: V A L \_33: C$ & - \\
\hline $1: \mathrm{GLU}-\_34: \mathrm{CA}$ & $1: \mathrm{GLU}-\_34: \mathrm{C}$ & - \\
\hline $1:$ ALA_35:CA & $1:$ ALA_35:C & - \\
\hline $1:$ LEU_36:CA & $1:$ LEU_36:C & - \\
\hline $1:$ TYR_37:CA & $1:$ TYR_37:C & - \\
\hline $1:$ LEU_38:CA & $1:$ LEU_38:C & - \\
\hline $1: \mathrm{VAL} 399: \mathrm{CA}$ & 1:VAL_39:C & - \\
\hline $1:$ CYS_4 $0: \mathrm{CA}$ & $1: C Y S_{-} 40: C$ & - \\
\hline $1:$ ILE_10:CA & $1:$ ILE_10:C & - \\
\hline $1: S E R \_12: C A$ & $1: S E R \_12: C$ & - \\
\hline $1: \mathrm{VAL} \_23: \mathrm{CA}$ & $1: \mathrm{VAL} \_23: \mathrm{C}$ & - \\
\hline $1:$ GLN_25:CA & $1: G L N \_25: C$ & - \\
\hline $1:$ LEU_27:CA & $1:$ LEU_27:C & - \\
\hline $1: \mathrm{PHE}_{-} 46: \mathrm{CA}$ & $1: \mathrm{PHE}_{-} 46: \mathrm{C}$ & - \\
\hline $1:$ THR_48:CA & $1:$ THR_48:C & - \\
\hline $1: \mathrm{GLU}_{-}{ }^{4} \mathrm{:}: \mathrm{CB}$ & $1: \mathrm{GLU}-\_4: \mathrm{CG}$ & - \\
\hline $1: \mathrm{ASN} \_18: \mathrm{CB}$ & $1:$ ASN_18:CG & - \\
\hline 1:TYR_19:CB & 1:TYR_19:CG & - \\
\hline $1:$ CYS_20:CB & $1:$ CYS_20:SG & - \\
\hline
\end{tabular}




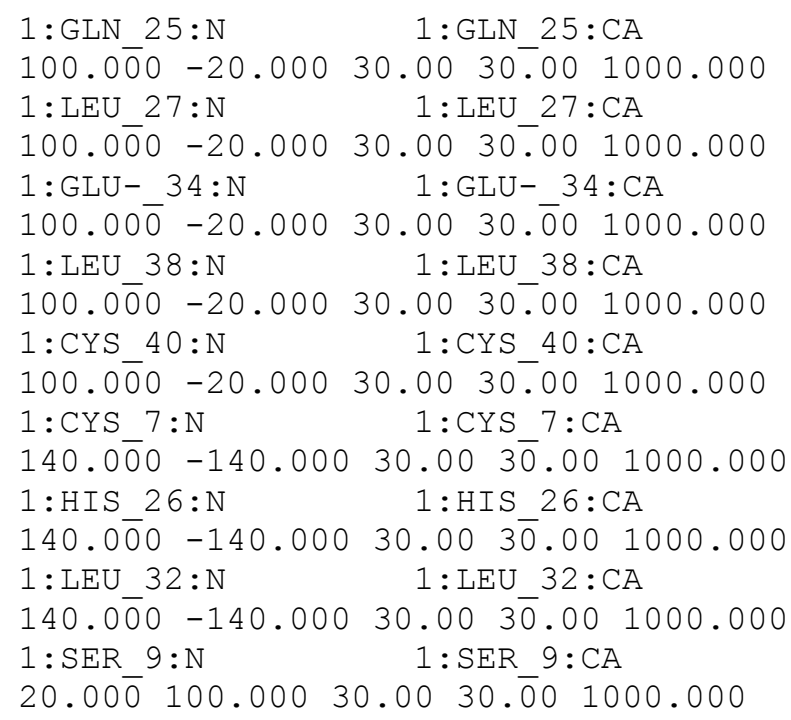

\begin{tabular}{|c|c|}
\hline $1: \mathrm{GLN}_{2} 25: \mathrm{CB}$ & $1: G L N \_25: C G$ \\
\hline $1: \mathrm{LEU} \_27: \mathrm{CB}$ & 1:LEU_27:CG \\
\hline $1: \mathrm{GLU}-\_34: \mathrm{CB}$ & $1: \mathrm{GLU}-\_34: \mathrm{CG}$ \\
\hline $1: \mathrm{LEU}_{2} 38: \mathrm{CB}$ & $1:$ LEU_38:CG \\
\hline $1: C Y S \_40: C B$ & $1:$ CYS_40:SG \\
\hline $1: \mathrm{CYS}_{-} 7: \mathrm{CB}$ & $1: \mathrm{CYS}_{-} 7: \mathrm{SG}$ \\
\hline $1: \mathrm{HIS}_{2} 26: \mathrm{CB}$ & $1: H I S \_26: C G$ \\
\hline $1: \mathrm{LEU}_{-} 32: \mathrm{CB}$ & $1: \mathrm{LEU} \_32: \mathrm{CG}$ \\
\hline $1:$ SER_9:CB & $1:$ SER_9:OG \\
\hline
\end{tabular}

